\title{
103. THE PETROLOGY AND GEOCHEMISTRY OF BASALTS FROM SITE 396, LEGS 45 AND 46 OF THE DEEP SEA DRILLING PROJECT
}

\author{
M. A. Dungan, ${ }^{1}$ J. M. Rhodes,${ }^{2}$ P. E. Long, ${ }^{1}$ D. P. Blanchard,${ }^{3}$ J. C. Brannon, ${ }^{2}$ and K. V. Rodgers ${ }^{2}$
}

\begin{abstract}
Legs 45 and 46 of the Deep Sea Drilling Project sampled a total of 351 meters of basaltic basement at Site 396. Several compositionally distinct eruptive units are found at this site, each having the characteristics of evolved mid-ocean ridge tholeiite. The minor and trace element abundances of the Site 396 basalts are also typical of LREE-depleted mid-ocean ridge tholeiite. Basalts sampled include aphyric and phyric basalts, the latter containing abundant plagioclase and olivine phenocrysts. Microprobe analyses of olivine microphenocrysts and coexisting glass in both basalt types yield a $\mathrm{KD}=0.27$ for $\mathrm{Fe}-\mathrm{Mg}$ partitioning between olivine and liquid. Application of this KD to phenocryst-bulk rock pairs indicates that many of the phenocrysts are more magnesian than the presumed equilibrium composition and must have been derived from a more primitive magma. One-atmosphere melting studies confirm this discrepancy. The diversity of zoning patterns in the plagioclase phenocrysts in the phyric basalts similarly indicates a mixed parentage for this phase. In addition, both the olivine and plagioclase phenocrysts contain $\mathrm{Mg}$-rich, Ca-rich, Ti-poor melt inclusions which have compositional characteristics of more "primitive" magmas. Integration of these observations with major and trace element chemical data indicates that mixing of magmas and their attendant phenocrysts played an important role in the generation of these basalts. We suggest that mixing occurs as "primitive" picritic magmas are injected into zoned, fractionating magma chambers.

The basalts recovered from Site 395 are very similar to the Site 396 lavas in mineralogy, mineral chemistry, and bulk composition. The nearly identical trace element systematics of basalts from the two sites indicate derivation of these LIL-depleted magmas from a similar, compositionally homogeneous mantle source.
\end{abstract}

\section{INTRODUCTION}

A total of 351 meters of volcanic basement was sampled during Legs 45 and 46 at Site 396 (Hole $396=96 \mathrm{~m}$; Hole $396 \mathrm{~B}=355 \mathrm{~m})$, the vast majority of which consists of basaltic pillow lava. Hole 396B also includes a thin cooling unit $(9 \mathrm{~m})$ believed to be a single massive flow. Drilling in Hole 396B was terminated after the caving-in of a clastic unit comprised of fragments of pillow basalt. Emphasis in this paper has been placed on basalts from Hole 396B although data from Hole 396 are included.

We report 103 major element analyses on selected samples from Holes 396B and 396; 94 analyses of the minor and trace elements $\mathrm{Rb}, \mathrm{Sr}, \mathrm{Y}, \mathrm{Zr}$, and $\mathrm{Nb}$, and 28 analyses of $\mathrm{La}, \mathrm{Ce}, \mathrm{Sm}, \mathrm{Eu}, \mathrm{Tb}, \mathrm{Yb}, \mathrm{Lu}, \mathrm{Hf}, \mathrm{Cr}, \mathrm{Sc}$, and Ni. These data are listed in Tables 1 and 2. Some of the individual rock samples obtained by onboard sampling were split into two sub-samples of either relatively fresh and altered

${ }^{1}$ Geology Branch, NASA Johnson Space Center, Houston, Texas.

${ }^{2}$ Lockheed Electronics Co., Houston, Texas.

${ }^{3}$ Geochemistry Branch, NASA Johnson Space Center, Houston, Texas. material or the glassy rind which was separated from the adjacent crystalline material (see Table 1). Where samples listed in Table 2 have multiple sub-samples, the material analyzed by neutron activation was the glassy or fresh split. Samples are listed throughout the tables in order of increasing depth, with the exception of JSC No. 215 (396B-15-5, 58-63 cm) which in fact belongs stratigraphically between JSC Nos. 221 and 222 . Also indicated in Tables 1 and 2 are the major magmatic units, which were initially identified on the basis of shipboard $\mathrm{X}$-ray fluorescence analyses, and modified slightly in the light of newly acquired data. The oxidation ratio (O.R.) in Table 1 is given as $\mathrm{Fe}^{2} \mathrm{O}_{3} /($ total iron as $\mathrm{FeO})$. The atomic ratio $\mathrm{Mg} /\left(\mathrm{Mg}+\mathrm{Fe}^{+2}\right)$ is given as $\mathrm{Mg}^{\prime}$ in this table and has been calculated after adjustment to a fixed oxidation state of iron such that $\mathrm{Fe}^{+3} /\left(\right.$ total iron as $\left.\mathrm{Fe}^{+2}\right)=0.1$ (Bass, 1971). The same convention for $\mathrm{Fe}^{+3} /\left(\mathrm{Fe}^{+2}+\mathrm{Fe}^{+3}\right)$ has been used in the CIPW norm calculations that are referred to in the text and illustrated in Figure 1. Table 3 is a listing of average compositions of the five chemically defined units in Hole 396B. The averages were calculated after exclusion of analyses of highly altered rocks (O.R. $>0.4, \mathrm{H}_{2} \mathrm{O}>2.0$, and obvious loss of $\mathrm{MgO}$ relative to fresh samples). 
TABLE 1

Major and Trace Element Analyses of Basalts From Hole 396B, DSDP Leg 46

\begin{tabular}{|c|c|c|c|c|c|c|c|c|c|c|c|c|c|c|}
\hline $\begin{array}{c}\text { Sample } \\
\text { (Interval in } \mathrm{cm} \text { ) }\end{array}$ & $\begin{array}{c}4-1 \\
119-122\end{array}$ & $\begin{array}{r}4-2 \\
75-75\end{array}$ & $\begin{array}{c}6-1 \\
73-78\end{array}$ & $\begin{array}{r}7-1 \\
10-14\end{array}$ & $\begin{array}{r}7-1 \\
10-14\end{array}$ & $\begin{array}{r}7-1 \\
42-46\end{array}$ & $\begin{array}{r}7-1 \\
50-57\end{array}$ & $\begin{array}{c}7-2 \\
16-20\end{array}$ & $\begin{array}{c}7-2 \\
70-77\end{array}$ & $\begin{array}{c}7-2 \\
118-122\end{array}$ & $\begin{array}{c}7-2 \\
118-122\end{array}$ & $\begin{array}{r}8-1 \\
76-82\end{array}$ & $\begin{array}{r}8-1 \\
76-82\end{array}$ & $\begin{array}{r}8-1 \\
84-94\end{array}$ \\
\hline JSC No. & 180 & 181 & 182 & 183 & 183 & 184 & $185(\mathrm{Std})$ & 187 & 188 & 189 & 189 & 190 & 190 & 191 \\
\hline Unit & $A_{1}$ & $A_{1}$ & $A_{1}$ & $A_{1}$ & $A_{1}$ & $A_{1}$ & $A_{1}$ & $\mathrm{~A}_{1}$ & $A_{1}$ & $A_{1}$ & $A_{1}$ & $\mathrm{~A}_{2}$ & $\mathrm{~A}_{2}$ & $\mathrm{~A}_{2}$ \\
\hline Basalt Type & & & & Glass & & & & & & Fresh & Altered & Rock & Glass & Fresh \\
\hline $\mathrm{SiO}_{2}$ & 50.01 & 48.03 & 50.22 & 49.32 & 49.52 & 48.81 & 49.24 & 49.23 & 49.03 & 49.10 & 48.87 & 49.18 & 49.78 & 49.26 \\
\hline $\mathrm{TiO}_{2}$ & 1.42 & 1.47 & 1.41 & 1.40 & 1.40 & 1.42 & 1.40 & 1.44 & 1.40 & 1.39 & 1.38 & 1.56 & 1.51 & 1.53 \\
\hline $\mathrm{Al}_{2} \tilde{\mathrm{O}}_{3}$ & 15.40 & 15.60 & 15.50 & 15.37 & 15.53 & 15.62 & 15.46 & 15.70 & 15.32 & 15.30 & 15.44 & 15.90 & 15.10 & 15.59 \\
\hline $\mathrm{Fe}_{2} \mathrm{O}_{3}$ & 2.10 & 2.52 & 4.54 & 1.75 & 3.30 & 3.81 & 3.58 & 2.74 & 4.19 & 3.12 & 3.71 & 2.22 & 4.64 & 4.59 \\
\hline $\mathrm{FeO}$ & 7.40 & 6.15 & 4.67 & 7.61 & 6.26 & 5.90 & 5.98 & 6.99 & 5.51 & 6.06 & .5 .69 & 7.84 & 5.32 & 5.51 \\
\hline $\mathrm{MnO}$ & 0.17 & 0.19 & 0.18 & 0.16 & 0.21 & 0.20 & 0.16 & 0.20 & 0.21 & 0.16 & 0.19 & 0.21 & 0.17 & 0.21 \\
\hline $\mathrm{MgO}$ & 7.93 & 8.03 & 6.14 & 7.92 & 7.98 & 7.42 & 7.72 & 5.40 & 6.10 & 8.01 & 7.43 & 6.66 & 7.86 & 6.94 \\
\hline $\mathrm{CaO}$ & 10.82 & 11.58 & 11.50 & 11.26 & 11.30 & 11.64 & 11.63 & 11.79 & 11.44 & 11.76 & 12.08 & 11.62 & 10.73 & 11.50 \\
\hline $\mathrm{Na}_{2} \mathrm{O}$ & 2.71 & 2.70 & 2.75 & 2.73 & 2.70 & 2.67 & 2.54 & 2.68 & 2.58 & & & & & \\
\hline $\mathrm{K}_{2} \mathrm{O}$ & 0.28 & 0.22 & 0.25 & 0.16 & 0.16 & 0.17 & 0.18 & 0.14 & 0.24 & 0.23 & 0.28 & 0.15 & 0.20 & 0.19 \\
\hline $\mathrm{P}_{2} \mathrm{O}_{5}$ & 0.12 & 0.12 & 0.12 & 0.10 & 0.10 & 0.11 & 0.11 & 0.12 & 0.11 & 0.12 & 0.11 & 0.15 & 0.12 & 0.12 \\
\hline $\mathrm{s}$ & 0.11 & 0.07 & 0.02 & 0.09 & 0.11 & 0.07 & 0.06 & 0.07 & 0.04 & 0.03 & 0.04 & 0.06 & 0.11 & 0.05 \\
\hline Total $\mathrm{H}_{2} \mathrm{O}$ & 1.02 & 1.53 & 2.52 & 1.40 & 1.95 & 1.63 & 1.64 & 1.52 & 2.09 & 1.76 & 1.92 & 1.52 & 1.94 & 2.00 \\
\hline Total & 99.49 & 100.06 & 99.82 & 99.27 & 100.56 & 99.47 & 99.70 & 98.02 & 98.26 & 99.80 & 99.82 & 99.84 & 100.30 & 100.28 \\
\hline Total $\mathrm{Fe}$ as $\mathrm{FeO}$ & 9.29 & 9.32 & 8.76 & 9.18 & 9.23 & 9.33 & 9.20 & 9.46 & 9.28 & 8.87 & 9.03 & 9.84 & 9.50 & 9.64 \\
\hline Mg' value & 0.628 & 0.630 & 0.581 & 0.631 & 0.631 & 0.612 & 0.624 & 0.531 & 0.566 & 0.641 & 0.620 & 0.573 & 0.621 & 0.588 \\
\hline O.R. & 0.226 & 0.364 & 0.493 & 0.187 & 0.345 & 0.392 & 0.374 & 0.282 & 0.432 & 0.352 & 0.395 & 0.221 & 0.466 & 0.454 \\
\hline \multicolumn{15}{|l|}{$\begin{array}{l}\text { Trace elements } \\
\text { (ppm) }\end{array}$} \\
\hline $\mathrm{Rb}$ & 1.4 & 1.6 & 4.0 & & 0.8 & 0.9 & 1.8 & 1.0 & 3.2 & 2.7 & & 0.3 & & 2.1 \\
\hline $\mathrm{Sr}$ & 119 & 122 & 120 & & 122 & 122 & 121 & 123 & 119 & 122 & & 132 & & 131 \\
\hline $\mathrm{Y}$ & 30.2 & 31.6 & 30.2 & & 31.7 & 31.5 & 30.2 & 30.9 & 28.9 & 29.5 & & 34.8 & & 33.9 \\
\hline $\mathrm{Zr}$ & 96 & 93 & 93 & & 93 & 93 & 94 & 93 & 93 & 87 & & 103 & & 103 \\
\hline $\begin{array}{l}\mathrm{Nb} \\
\mathrm{Ni}\end{array}$ & 1.7 & 1.9 & 2.0 & & 1.8 & 1.7 & 2.1 & 1.1 & 1.8 & 1.6 & & 1.5 & & 2.9 \\
\hline
\end{tabular}

TABLE 1 - Continued

\begin{tabular}{|c|c|c|c|c|c|c|c|c|c|c|c|c|c|c|}
\hline $\begin{array}{c}\text { Sample } \\
\text { (Interval in } \mathrm{cm} \text { ) }\end{array}$ & $\begin{array}{l}13-1 \\
30-33\end{array}$ & $\begin{array}{c}13-1 \\
102-105\end{array}$ & $\begin{array}{c}13-1, \\
102-105\end{array}$ & $\begin{array}{c}13-2, \\
21-25\end{array}$ & $\begin{array}{r}13-2 \\
57-58\end{array}$ & $\begin{array}{c}13-2 \\
130-134\end{array}$ & $\begin{array}{c}\text { 13-2 } \\
137-140\end{array}$ & $\begin{array}{l}13-3, \\
3-5\end{array}$ & $\begin{array}{l}14-1 \\
9-13\end{array}$ & $\begin{array}{l}14-1 \\
9-13\end{array}$ & $\begin{array}{l}14-1, \\
21-25\end{array}$ & $\begin{array}{r}14-2, \\
39-46\end{array}$ & $\begin{array}{r}14-2 \\
57-61\end{array}$ & $\begin{array}{r}15-2 \\
54-61\end{array}$ \\
\hline JSC No. & 201 & 202 & 202 & 203 & 204 & 205 & 206 & 207 & 208 & 208 & 209 & 210 & 211 & 212 \\
\hline Unit & $\mathrm{A}_{2}$ & $\mathrm{~A}_{2}$ & $\mathrm{~A}_{2}$ & $\mathrm{~A}_{2}$ & $\mathrm{~A}_{2}$ & $\mathrm{~A}_{2}$ & $\mathrm{~A}_{2}$ & $\mathrm{~A}_{2}$ & $\mathrm{~A}_{2}$ & $\mathrm{~A}_{2}$ & $\mathrm{~A}_{2}$ & $A_{2}$ & $\mathrm{~A}_{2}$ & $A_{2}$ \\
\hline Basalt Type & & Glass & Rock & Glass & & & & & Glass & Rock & & & & \\
\hline $\mathrm{SiO}_{2}$ & 50.16 & 49.39 & 48.88 & 48.92 & 50.28 & 49.38 & 50.17 & 49.18 & 49.37 & 49.01 & 49.24 & 49.26 & 48.64 & 48.62 \\
\hline $\mathrm{TiO}_{2}$ & 1.51 & 1.56 & 1.51 & 1.54 & 1.51 & 1.51 & 1.50 & 1.63 & 1.62 & 1.62 & 1.64 & 1.62 & 1.64 & 1.58 \\
\hline $\mathrm{Al}_{2} \mathrm{O}_{3}$ & 15.22 & 15.70 & 15.10 & 15.03 & 15.26 & 15.31 & 15.20 & 15.20 & 15.10 & 1.546 & 15.44 & 15.12 & 15.27 & 14.74 \\
\hline $\mathrm{Fe}_{2} \mathrm{O}_{3}$ & 2.60 & 1.42 & 3.03 & 2.42 & 3.39 & 2.97 & 2.16 & 5.28 & 1.19 & 2.63 & 3.39 & 3.88 & 3.10 & 5.08 \\
\hline $\mathrm{FeO}$ & 7.14 & 8.45 & 6.89 & 7.54 & 5.83 & 6.85 & 7.50 & 4.55 & 8.82 & 7.55 & 6.97 & 6.29 & 7.25 & 5.63 \\
\hline $\mathrm{MnO}$ & 0.19 & 0.21 & 0.18 & 0.19 & 0.19 & 0.20 & 0.17 & 0.16 & 0.18 & 0.19 & 0.20 & 0.19 & 0.20 & 0.20 \\
\hline $\mathrm{MgO}$ & 8.00 & 7.12 & 7.81 & 7.12 & 7.60 & 7.82 & 7.84 & 7.04 & 7.66 & 7.38 & 7.17 & 7.30 & 7.27 & 6.96 \\
\hline $\mathrm{CaO}$ & 11.22 & 11.44 & 10.22 & 10.94 & 11.14 & 11.19 & 11.12 & 10.80 & 10.65 & 10.84 & 10.86 & 10.69 & 10.84 & 11.15 \\
\hline $\mathrm{Na}_{2} \mathrm{O}$ & 2.71 & 2.85 & 2.78 & 2.77 & 2.87 & 2.85 & 2.83 & 3.11 & 2.94 & 2.98 & 3.10 & 2.98 & 3.06 & 3.14 \\
\hline $\mathrm{K}_{2} \mathrm{O}$ & 0.22 & 0.17 & 0.38 & 0.16 & 0.25 & 0.22 & 0.14 & 0.27 & 0.15 & 0.22 & 0.26 & 0.37 & 0.27 & 0.29 \\
\hline $\mathrm{P}_{2} \mathrm{O}_{5}$ & 0.14 & 0.14 & 0.10 & 0.14 & 0.14 & 0.10 & 0.11 & 0.12 & 0.15 & 0.14 & 0.16 & 0.14 & 0.15 & 0.15 \\
\hline $\mathrm{s}$ & 0.08 & 0.08 & 0.10 & 0.08 & 0.03 & 0.06 & 0.10 & 0.00 & 0.11 & 0.08 & 0.07 & 0.03 & 0.06 & 0.02 \\
\hline Total $\mathrm{H}_{2} \mathrm{O}$ & 1.47 & 1.53 & 2.40 & 1.40 & 2.47 & 1.97 & 1.21 & 2.52 & 0.98 & 1.32 & 1.68 & 1.86 & 1.40 & 2.09 \\
\hline Total & 100.66 & 100.06 & 99.37 & 98.25 & 100.96 & 100.43 & 100.06 & 99.85 & 98.92 & 99.41 & 100.18 & 99.73 & 99.15 & 99.65 \\
\hline Total Fe as FeO & 9.48 & 9.73 & 9.62 & 9.72 & 8.88 & 9.52 & 9.44 & 9.30 & 9.89 & 9.92 & 10.02 & 9.79 & 10.04 & 10.20 \\
\hline Mg'value & 0.626 & 0.592 & 0.6116 & 0.592 & 0.629 & 0.619 & 0.622 & 0.600 & 0.618 & 0.596 & 0.586 & 0.596 & 0.589 & 0.575 \\
\hline O.R. & 0.267 & 0.144 & 0.305 & 0.243 & 0.368 & 0.302 & 0.224 & 0.537 & 0.120 & 0.258 & 0.327 & 0.382 & 0.300 & 0.474 \\
\hline \multicolumn{15}{|l|}{$\begin{array}{l}\text { Trace elements } \\
(\mathrm{ppm})\end{array}$} \\
\hline $\mathrm{Rb}$ & 1.3 & & 1.1 & 0.7 & 2.8 & 1.6 & 0.8 & 3.9 & 1.4 & 1.3 & & 4.5 & 2.0 & 4.3 \\
\hline Sr & 121 & & 131 & 125 & 128 & 125 & 125 & 149 & 133 & 142 & & 134 & 138 & 151 \\
\hline $\mathrm{Y}$ & 33.1 & & 34.1 & 31.9 & 33.1 & 32.6 & 33.1 & 35.2 & 35.2 & 36.3 & & 33.7 & 35.6 & 33.6 \\
\hline $\mathrm{Zr}_{\mathrm{r}}$ & 100 & & 105 & 104 & 103 & 101 & 102 & 118 & 120 & 119 & & 113 & 118 & 112 \\
\hline $\begin{array}{l}\mathrm{Nb} \\
\mathrm{Ni}\end{array}$ & 2.0 & & 2.1 & 2.3 & 1.7 & 2.4 & 1.5 & 2.3 & 2.1 & 2.2 & & 1.7 & 1.9 & 1.7 \\
\hline
\end{tabular}


TABLE 1 - Continued

\begin{tabular}{|c|c|c|c|c|c|c|c|c|c|c|c|c|c|}
\hline $\begin{array}{r}8-1 \\
84-94\end{array}$ & $\begin{array}{c}9-2 \\
74-82\end{array}$ & $\begin{array}{r}9-2 \\
74-82\end{array}$ & $\begin{array}{c}9-3 \\
19-23\end{array}$ & $\begin{array}{l}\text { 10-1, } \\
96-101\end{array}$ & $\begin{array}{c}10-2 \\
14-18\end{array}$ & $\begin{array}{r}10-2 \\
14-18\end{array}$ & $\begin{array}{c}10-2, \\
29-33\end{array}$ & $\begin{array}{c}11-1, \\
70-73\end{array}$ & $\begin{array}{c}11-1 \\
78-86\end{array}$ & $\begin{array}{l}11-1, \\
78-86\end{array}$ & $\begin{array}{c}11-2, \\
36-40\end{array}$ & $\begin{array}{c}1 \mathrm{~T}-2 \text {, } \\
36-40\end{array}$ & $\begin{array}{c}12-1 \text {, } \\
117-120\end{array}$ \\
\hline 191 & 192 & 192 & 193 & 194 & 195 & 195 & 196 & 197 & 198 & 198 & 199 & 199 & 200 \\
\hline$A_{2}$ & $A_{2}$ & $\mathrm{~A}_{2}$ & $A_{2}$ & $A_{2}$ & $\mathrm{~A}_{2}$ & $A_{2}$ & $\mathrm{~A}_{2}$ & $A_{2}$ & $\mathrm{~A}_{2}$ & $\mathrm{~A}_{2}$ & $A_{2}$ & $A_{2}$ & $A_{2}$ \\
\hline Altered & Glass & Rock & & & Glass & & & & Fresh & Altered & Glass & & \\
\hline 48.51 & 49.90 & 49.46 & 49.21 & 49.42 & 50.49 & 50.19 & 49.58 & 48.88 & 49.20 & 49.12 & 50.39 & 49.67 & 49.98 \\
\hline 1.59 & 1.54 & 1.51 & 1.50 & 1.54 & 1.51 & 1.53 & 1.54 & 1.53 & 1.50 & 1.54 & 1.52 & 1.51 & 1.48 \\
\hline 16.00 & 15.38 & 15.00 & 15.01 & 15.36 & 14.80 & 15.33 & 15.43 & 15.55 & 14.96 & 15.46 & 15.29 & 15.31 & 15.13 \\
\hline 5.67 & 1.20 & 2.86 & 4.50 & 2.14 & 0.38 & 1.84 & 2.81 & 2.06 & 3.20 & 4.51 & 0.73 & 1.81 & 3.57 \\
\hline 4.79 & 8.52 & 6.92 & 4.92 & 7.65 & 9.09 & 7.87 & 7.08 & 7.73 & 6.58 & 5.50 & 8.84 & 7.81 & 5.85 \\
\hline 0.19 & 0.20 & 0.18 & 0.18 & 0.19 & 0.20 & 0.20 & 0.20 & 0.20 & 0.20 & 0.20 & 0.16 & 0.20 & 0.18 \\
\hline 6.06 & 7.33 & 7.77 & 7.60 & 7.69 & 7.86 & 7.75 & 7.28 & 7.42 & 7.68 & 6.26 & 8.17 & 7.83 & 7.65 \\
\hline 11.82 & 11.31 & 10.68 & 11.12 & 11.30 & 11.06 & 11.24 & 11.36 & 11.13 & 11.14 & 11.47 & 11.14 & 11.18 & 11.06 \\
\hline 2.89 & 2.68 & 2.79 & 2.81 & 2.74 & 2.73 & 2.68 & 2.74 & 2.84 & 2.78 & 2.94 & 2.78 & 2.67 & 2.88 \\
\hline 0.20 & 0.18 & 0.23 & 0.23 & 0.16 & 0.12 & 0.14 & 0.18 & 0.19 & 0.25 & 0.22 & 0.14 & 0.15 & 0.26 \\
\hline 0.14 & 0.14 & 0.09 & 0.10 & 0.11 & 0.10 & 0.12 & 0.13 & 0.11 & 0.13 & 0.14 & 0.12 & 0.12 & 0.15 \\
\hline 0.03 & 0.07 & 0.10 & 0.00 & 0.08 & 0.11 & 0.08 & 0.06 & 0.08 & 0.04 & 0.04 & 0.12 & 0.08 & 0.03 \\
\hline 2.43 & 1.44 & 1.60 & 2.66 & 1.62 & 0.49 & 1.69 & 1.49 & $2.60 \mathrm{f}$ & 1.94 & 2.72 & 0.74 & 1.30 & 2.53 \\
\hline 100.32 & 99.81 & 99.19 & 99.84 & 100.00 & 98.93 & 100.65 & 99.88 & 100.19 & 99.60 & 100.20 & 100.14 & 99.64 & 100.75 \\
\hline 9.89 & 9.60 & 9.49 & 8.97 & 9.58 & 9.43 & 9.53 & 9.61 & 9.58 & 9.46 & 9.56 & 9.50 & 9.44 & 9.06 \\
\hline 0.548 & 0.602 & 0.619 & 0.627 & 0.614 & 0.623 & 0.617 & 0.600 & 0.605 & 0.617 & 0.565 & 0.630 & 0.622 & 0.626 \\
\hline 0.542 & 0.123 & 0.286 & 0.478 & 0.219 & 0.040 & 0.189 & 0.284 & 0.210 & 0.327 & 0.450 & 0.076 & 0.188 & 0.379 \\
\hline 2.3 & & 1.0 & 3.1 & 1.0 & 0.9 & 1,0 & 1.2 & 1.4 & 3.3 & 2.9 & 0.9 & 0.9 & 3.2 \\
\hline 133 & & 127 & 144 & 127 & 114 & 123 & 129 & 130 & 124 & 131 & 120 & 125 & 133 \\
\hline 34.0 & & 32.5 & 32.3 & 33.2 & 31.3 & 32.4 & 33.7 & 32.9 & 32.3 & 33.9 & 25.3 & 33.7 & 31.1 \\
\hline 107 & & 103 & 102 & 105 & 99 & 102 & 104 & 105 & 101 & 106 & 104 & 104 & 98 \\
\hline 1.5 & & 2.2 & 1.8 & 2.1 & 2.0 & 2.4 & 2.1 & 2.0 & 1.5 & 1.8 & 1.9 & 2.0 & 2.2 \\
\hline
\end{tabular}

TABLE 1 - Continued

\begin{tabular}{|c|c|c|c|c|c|c|c|c|c|c|c|c|c|}
\hline $\begin{array}{c}15-2, \\
106-111\end{array}$ & $\begin{array}{c}15-2 \\
120-133\end{array}$ & $\begin{array}{l}15-5 \\
58-63\end{array}$ & $\begin{array}{r}15-3 \\
25-33\end{array}$ & $\begin{array}{r}15-3 \\
25-33\end{array}$ & $\begin{array}{l}15-3 \\
40-51\end{array}$ & $\begin{array}{c}15-3 \\
112-117\end{array}$ & $\begin{array}{l}15-4 \\
20-26\end{array}$ & $\begin{array}{r}15-4, \\
85-90\end{array}$ & $\begin{array}{c}15-4 \\
142-149\end{array}$ & $\begin{array}{l}15-5 \\
80-85\end{array}$ & $\begin{array}{c}16-1 \\
20-24\end{array}$ & $\begin{array}{c}16-1 \text {, } \\
50-55\end{array}$ & $\begin{array}{l}16-2 \\
3-16\end{array}$ \\
\hline 213 & 214 (Std) & 215 & 216 & 216 & 217 & 218 & 219 & 220 & 221 & 222 & 223 & 224 & 225 \\
\hline \multirow[t]{2}{*}{$\mathrm{A}_{3}$} & $\mathrm{~A}_{3}$ & $\mathrm{~A}_{3}$ & $\mathrm{~A}_{3}$ & $A_{3}$ & $\mathrm{~A}_{3}$ & $\mathrm{~A}_{3}$ & $\mathrm{~A}_{3}$ & $\mathrm{~A}_{3}$ & $\mathrm{~A}_{3}$ & $\mathrm{~A}_{3}$ & B & B & B \\
\hline & & & Fresh & Altered & & & & & & & & & Fresh \\
\hline 48.78 & 49.02 & 49.03 & 49.36 & 48.98 & 49.06 & 47.97 & 49.30 & 49.30 & 49.24 & 48.79 & 49.51 & 47.22 & 48.46 \\
\hline 1.60 & 1.61 & 1.56 & 1.59 & 1.61 & 1.61 & 1.59 & 1.60 & 1.57 & 1.60 & 1.60 & 1.23 & 1.21 & 1.19 \\
\hline 14.97 & 14.97 & 15.06 & 14.84 & 14.94 & 15.36 & 15.30 & 15.05 & 15.05 & 14.98 & 15.04 & 16.74 & 17.49 & 16.75 \\
\hline 2.39 & 2.20 & 4.26 & 2.69 & 5.14 & 5.13 & 5.57 & 2.40 & 2.76 & 2.78 & 4.52 & 3.88 & 4.02 & 3.37 \\
\hline 7.60 & 7.68 & 5.76 & 6.95 & 5.09 & 5.14 & 4.77 & 7.60 & 7.18 & 7.04 & 5.52 & 4.71 & 4.99 & 5.16 \\
\hline 0.18 & 0.15 & 0.20 & 0.19 & 0.19 & 0121 & 0.21 & 0.19 & 0.17 & 0.20 & 0.20 & 0.19 & 0.19 & 0.18 \\
\hline 7.94 & 7.96 & 7.38 & 7.50 & 7.22 & 6.84 & 5.68 & 7.51 & 7.82 & 7.45 & 7.33 & 6.60 & 6.58 & 7.47 \\
\hline 10.56 & 10.58 & 10.52 & 10.46 & 10.59 & 10.70 & 11.72 & 10.55 & 10.52 & 10.57 & 10.59 & 11.98 & 12.16 & 11.83 \\
\hline 3.10 & 2.97 & 2.96 & 2.89 & 2.85 & 2.92 & 3.12 & 2.89 & 2.85 & 2.75 & 2.91 & 2.66 & 2.65 & 2.60 \\
\hline 0.12 & 0.12 & 0.31 & 0.14 & 0.26 & 0.27 & 0.22 & 0.16 & 0.13 & 0.19 & 0.28 & 0.19 & 0.15 & 0.22 \\
\hline 0.13 & 0.13 & 0.12 & 0.14 & 0.16 & 0.15 & 0.14 & 0.15 & 0.14 & 0.15 & 0.12 & 0.09 & 0.08 & 0.09 \\
\hline 0.11 & 0.12 & 0.011 & 0.15 & 0.03 & 0.01 & 0.00 & 0.13 & 0.12 & 0.13 & 0.02 & 0.00 & 0.02 & 0.02 \\
\hline 1.98 & 2.35 & 2.27 & 2.42 & 2.29 & 2.52 & 2.54 & 2.16 & 2.05 & 2.38 & 2.31 & 2.47 & 1.78 & 2.00 \\
\hline 99.46 & 99.87 & 99.46 & 99.33 & 99.35 & 99.92 & 98.83 & 99.68 & 99.67 & 99.46 & 99.30 & 100.25 & 98.54 & 99.34 \\
\hline 9.75 & 9.66 & 9.60 & 9.37 & 9.72 & 9.76 & 9.78 & 9.76 & 9.66 & 9.54 & 9.59 & 8.20 & 8.61 & 8.19 \\
\hline 0.617 & 0.620 & 0.604 & 0.613 & 0.595 & 0.581 & 0.535 & 0.604 & 0.616 & 0.607 & 0.602 & 01615 & 0.602 & 0.644 \\
\hline 0.239 & 0.223 & 0.425 & 0.279 & 0.502 & 0.500 & 0.539 & 0.240 & 0.278 & 0.283 & 0.450 & 0.452 & 0.446 & 0.395 \\
\hline 1.0 & 0.9 & 3.7 & & 3.6 & 0.4 & 3.5 & 1.9 & 1.2 & 1.7 & 3.4 & 2.7 & 1.5 & 2.5 \\
\hline 136 & 136 & 142 & & 143 & 145 & 152 & 135 & 137 & 134 & 148 & 127 & 132 & 126 \\
\hline 35.0 & 35.2 & 34.3 & & 35.3 & 34.1 & 33.0 & 34.8 & 32.9 & 33.9 & 35.8 & 26.0 & 24.5 & 24.5 \\
\hline 117 & 116 & 112 & & 113 & 114 & 111 & 116 & 109 & 114 & 119 & 77 & 78 & 81 \\
\hline 2.7 & 1.5 & 2.5 & & 2.5 & 1.9 & 1.8 & 1.7 & 1.6 & 2.1 & 1.6 & 1.1 & 1.2 & 0.8 \\
\hline
\end{tabular}


TABLE 1 - Continued

\begin{tabular}{|c|c|c|c|c|c|c|c|c|c|c|c|c|c|c|}
\hline $\begin{array}{c}\text { Sample } \\
\text { (Interval in } \mathrm{cm} \text { ) }\end{array}$ & $\begin{array}{l}16-2 \\
3-16\end{array}$ & $\begin{array}{r}16-3 \\
46-51\end{array}$ & $\begin{array}{r}16-3 \\
46-51\end{array}$ & $\begin{array}{c}16-5 \\
130-134\end{array}$ & $\begin{array}{r}17-3 \\
73-81\end{array}$ & $\begin{array}{l}\text { 17-3 } \\
73-81\end{array}$ & $\begin{array}{l}17-4 \\
4-11\end{array}$ & & $\begin{array}{l}18-1 \\
3-11\end{array}$ & $\begin{array}{c}18-2 \\
124-131\end{array}$ & $\begin{array}{l}18-2 \\
9-14\end{array}$ & $\begin{array}{l}20-1, \\
61-64\end{array}$ & $\begin{array}{c}20-1 \\
102-104\end{array}$ & $\begin{array}{c}20-1, \\
136-138\end{array}$ \\
\hline JSC No. & 225 & 226 & 226 & 227 & 228 & 228 & 229 & 230 & 231 & 232 & 233 & 234 & 235 & 236 \\
\hline Unit & B & B & B & B & B & B & B & $\mathrm{B}_{1}$ & B & B & B & B & B & B \\
\hline Basalt Type & Altered & Glass & & & Fresh & Altered & & & & & & & & Glass \\
\hline $\mathrm{SiO}_{2}$ & 46.69 & 49.46 & 48.27 & 48.67 & 48.02 & 47.76 & 46.72 & 49.59 & 49.13 & 48.96 & 49.02 & 48.87 & 49.22 & 49.28 \\
\hline $\mathrm{TiO}_{2}$ & 1.29 & 1.23 & 1.21 & 1.17 & 1.24 & 1.30 & 1.28 & 1.20 & 1.18 & 1.16 & 1.17 & 1.12 & 1.14 & 1.13 \\
\hline $\mathrm{Al}_{2} \mathrm{O}_{3}$ & 17.96 & 16.70 & 17.46 & 16.43 & 17.88 & 18.71 & 18.46 & 16.93 & 16.81 & 16.78 & $16.7-$ & 16.78 & 17.24 & 17.29 \\
\hline $\mathrm{Fe}_{2} \mathrm{O}_{3}$ & 5.20 & 1.06 & 3.46 & 3.69 & 3.88 & 5.57 & 5.70 & 3.72 & 4.23 & 1.44 & 3.69 & 3.46 & 3.24 & 1.23 \\
\hline $\mathrm{FeO}$ & 4.32 & 7.55 & 5.30 & 4.82 & 5.07 & 4.00 & 3.74 & 4.70 & 4.33 & 6.74 & 4.83 & 4.85 & 4.88 & 6.98 \\
\hline $\mathrm{MnO}$ & 0.21 & 0.16 & 0.18 & 0.15 & 0.19 & 0.19 & 0.21 & 0.16 & 0.17 & 0.14 & 0.18 & 0.18 & 0.18 & 0.15 \\
\hline $\mathrm{MgO}$ & 4.82 & 7.66 & 6.70 & 7.20 & 6.33 & 4.77 & 4.23 & 7.15 & 7.38 & 7.16 & 7.18 & 7.48 & 7.07 & 7.70 \\
\hline $\mathrm{CaO}$ & 12.50 & 11.54 & 12.04 & 11.74 & 12.31 & 12.63 & 12.62 & 11.98 & 11.76 & 11.82 & 11.76 & 11.82 & 11.94 & 11.46 \\
\hline $\mathrm{Na}_{2} \mathrm{O}$ & 2.80 & 2.64 & 2.69 & 2.62 & 2.67 & 2.73 & 2.85 & - & 2.72 & 2.51 & 2.63 & 2.64 & 2.71 & 2.58 \\
\hline $\mathrm{K}_{2} \mathrm{O}$ & 0.16 & 0.17 & 0.16 & 0.16 & 0.14 & 0.15 & 0.24 & 0.21 & 0.20 & 0.08 & 0.19 & 0.21 & 0.21 & 0.21 \\
\hline $\mathrm{P}_{2} \mathrm{O}_{5}$ & 0.12 & 0.07 & 0.10 & 0.08 & 0.07 & 0.17 & 0.13 & 0.11 & 0.09 & 0.10 & 0.09 & 0.07 & 0.11 & 0.08 \\
\hline $\mathrm{s}$ & 0.00 & 0.10 & 0.04 & 0.02 & 0.04 & 0.02 & 0.00 & 0.02 & 0.02 & 0.12 & 0.02 & 0.00 & 0.03 & 0.10 \\
\hline Total $\mathrm{H}_{2} \mathrm{O}$ & 2.40 & 1.03 & 1.82 & 2.18 & 1.54 & 2.26 & 2.95 & 2.25 & 2.27 & 2.04 & 2.07 & 2.31 & 2.27 & 1.49 \\
\hline Total & 98.51 & 99.37 & 99.43 & 98.93 & 99.37 & 100.26 & 99.13 & & 100.28 & 99.05 & 99.54 & 99.79 & 100.24 & 99.68 \\
\hline Total $\mathrm{Fe}$ as $\mathrm{FeO}$ & 9.00 & 8.50 & 8.41 & 8.14 & 8.56 & 9.01 & 8.87 & 8.05 & 8.14 & 8.04 & 8.15 & 7.96 & 7.80 & 8.09 \\
\hline $\mathrm{Mg}^{\prime}$ value & 0.515 & 0.641 & 0.612 & 0.637 & 0.594 & 0.511 & 0.486 & 0.638 & 0.642 & 0.638 & 0.636 & 0.650 & 0.642 & 0.653 \\
\hline O.R. & 0.546 & 0.123 & 0.395 & 0.434 & 0.434 & 0.582 & 0.604 & 0.442 & 0.494 & 0.176 & 0.433 & 0.416 & 0.399 & 0.150 \\
\hline \multicolumn{15}{|l|}{$\begin{array}{l}\text { Trace elements } \\
(\mathrm{ppm})\end{array}$} \\
\hline $\mathrm{Rb}$ & 2.6 & & 1.2 & 2.3 & 1.1 & 1.1 & 2.6 & 2.9 & 2.5 & 0.9 & 2.4 & 3.1 & 2.8 & 1.7 \\
\hline Sr & 131 & & 127 & 127 & 130 & 137 & 135 & 132 & 127 & 123 & 129 & 131 & 126 & 127 \\
\hline $\mathrm{Y}$ & 27.7 & & 26.5 & 25.3 & 27.4 & 28.1 & 28.4 & 25.1 & 24.3 & 24.7 & 23.8 & 24.1 & 24.7 & 24.0 \\
\hline $\mathrm{Z}_{\mathrm{r}}$ & 77 & & 74 & 77 & 79 & 79 & 82 & 75 & 75 & 74 & 73 & 72 & 71 & 74 \\
\hline $\begin{array}{l}\mathrm{Nb} \\
\mathrm{Ni}\end{array}$ & 1.6 & & 1.4 & 1.0 & 1.5 & 1.3 & 1.8 & 2.4 & 1.3 & 1.1 & 1.5 & 1.0 & 1.1 & 1.0 \\
\hline
\end{tabular}

TABLE 1 - Continued

\begin{tabular}{|c|c|c|c|c|c|c|c|c|c|c|c|c|}
\hline $\begin{array}{c}\text { Sample } \\
\text { (Interval in } \mathrm{cm} \text { ) }\end{array}$ & $\begin{array}{r}22-2 \\
75-80\end{array}$ & $\begin{array}{r}22-3 \\
28-38\end{array}$ & $\begin{array}{l}22-3 \\
81-90\end{array}$ & $\begin{array}{c}22-3 \\
137-143\end{array}$ & $\begin{array}{r}23-1 \\
46-50\end{array}$ & $\begin{array}{l}24-1 \\
43-48\end{array}$ & $\begin{array}{r}24-1, \\
94-98\end{array}$ & $\begin{array}{l}30-1 \\
77-80\end{array}$ & $\begin{array}{l}32-1 \\
3-8\end{array}$ & $\begin{array}{r}32-1 \\
30-34\end{array}$ & $\begin{array}{l}32-1, \\
34-36\end{array}$ & $\begin{array}{l}32-1, \\
101-106\end{array}$ \\
\hline JSC No. & 248 & 249 (STD.) & 250 & 251 & 252 & 254 & 255 & 257 & 258 & 259 & 260 & 261 \\
\hline Unit & B & B & B & B & C & C & c & C & D & C & C & D \\
\hline
\end{tabular}

Basalt Type

\begin{tabular}{|c|c|c|c|c|c|c|c|c|c|c|c|c|}
\hline $\mathrm{SiO}_{2}$ & 48.63 & 48.24 & 47.44 & 48.88 & 48.11 & 49.39 & 47.92 & 50.69 & 50.36 & 48.59 & 49.80 & 48.71 \\
\hline $\mathrm{TiO}_{2}^{-}$ & 0.97 & 0.96 & 0.98 & 0.96 & 1.56 & 1.53 & 1.50 & 1.49 & 1.32 & 1.49 & 1.51 & 1.20 \\
\hline $\mathrm{Al}_{2} \mathrm{O}_{3}$ & 17.82 & 17.55 & 17.76 & 17.45 & 16.28 & 16.01 & 15.62 & 16.21 & 17.12 & 16.33 & 16.46 & 17.14 \\
\hline $\mathrm{Fe}_{2} \mathrm{O}_{3}$ & 3.08 & 2.96 & 3.13 & 1.79 & 4.53 & 4.46 & 3.09 & 1.58 & 2.46 & 3.28 & 3.26 & 3.20 \\
\hline $\mathrm{FeO}$ & 4.75 & 4.81 & 4.70 & 5.86 & 5.42 & 4.41 & 6.22 & 7.64 & 5.86 & 6.19 & 6.20 & 4.98 \\
\hline MnO & 0.18 & 0.13 & 0.17 & 0.18 & 0.19 & 0.20 & 0.18 & 0.19 & 0.20 & 0.15 & 0.17 & 0.17 \\
\hline $\mathrm{MgO}$ & 7.68 & 7.75 & 7.34 & 8.27 & 6.59 & 6.74 & 7.66 & 7.94 & 6.94 & 7.11 & 7.10 & 7.04 \\
\hline $\mathrm{CaO}$ & 11.89 & 12.12 & 12.00 & 12.20 & 11.26 & 11.29 & 11.22 & 11.24 & 11.74 & 11.46 & 11.52 & 11.64 \\
\hline $\mathrm{Na}_{2} \mathrm{O}$ & 2.57 & 2.43 & 2.47 & 2.48 & 2.90 & 2.92 & 2.78 & 2.82 & 2.79 & 2.74 & 2.79 & 2.75 \\
\hline $\mathrm{K}_{2} \mathrm{O}$ & 0.12 & 0.18 & 0.12 & 0.09 & 0.20 & 0.29 & 0.24 & 0.19 & 0.31 & 0.27 & 0.25 & 0.24 \\
\hline $\mathrm{P}_{2} \mathrm{O}_{5}$ & 0.11 & 0.07 & 0.05 & 0.08 & 0.16 & 0.14 & 0.11 & 0.12 & 0.14 & 0.13 & 0.14 & 0.11 \\
\hline $\mathrm{s}$ & 0.04 & 0.02 & 0.03 & $0 .-9$ & 0.03 & 0.00 & 0.03 & 0.09 & 0.05 & 0.08 & 0.09 & 0.01 \\
\hline Total $\mathrm{H}_{2} \mathrm{O}$ & 2.91 & 2.19 & 3.01 & 1.60 & 2.35 & 2.88 & 1.87 & 1.01 & 1.44 & 1.58 & 1.64 & 2.20 \\
\hline Total & 100.74 & 99,41 & 99.21 & 99.93 & 99.58 & 100.26 & 98.44 & 100.20 & 100.72 & 99.40 & 99.28 & 99.39 \\
\hline Total $\mathrm{Fe}$ as $\mathrm{FeO}$ & 7.52 & 7.47 & 7.52 & 7.47 & 9.50 & 8.42 & 9.00 & 9.06 & 8.07 & 9.14 & 9.13 & 7.86 \\
\hline Mg'value & 0.669 & 0.673 & 0.659 & 0.687 & 0.579 & 0.613 & 0.627 & 0.634 & 0.630 & 0.606 & 0.606 & 0.639 \\
\hline O.R. & 0.393 & 0.381 & 0.400 & 0.234 & 0.455 & 0.503 & 0.331 & 0.171 & 0.296 & 0.346 & 0.345 & 0.391 \\
\hline \multicolumn{13}{|l|}{$\begin{array}{l}\text { Trace elements } \\
(\mathrm{ppm})\end{array}$} \\
\hline $\mathrm{Rb}$ & 1.1 & 2.5 & 1.2 & 0.6 & 0.7 & 4.3 & 2.3 & & 3.1 & 1.7 & 1.5 & 3.3 \\
\hline St & 113 & 119 & 116 & 123 & 161 & 161 & 157 & & 152 & 156 & 154 & 158 \\
\hline $\mathrm{Y}$ & 20.7 & 21.0 & 21.1 & 20.3 & 35.5 & 32.2 & 31.4 & & 27.1 & 31.3 & 32.0 & 25.4 \\
\hline $\mathrm{Zr}$ & 57 & 59 & 60 & 58 & 110 & 111 & 109 & & 91 & 107 & 108 & 84 \\
\hline $\begin{array}{l}\mathrm{Nb} \\
\mathrm{Ni}\end{array}$ & 0.9 & 0.5 & 0.7 & 0.8 & 4.1 & 3.3 & 3.3 & & 2.9 & 2.9 & 3.8 & 3.4 \\
\hline
\end{tabular}


TABLE 1 - Continued

\begin{tabular}{|c|c|c|c|c|c|c|c|c|c|c|c|c|c|}
\hline $\begin{array}{c}20-1 \\
136-138\end{array}$ & $\begin{array}{l}20-2 \\
58-67\end{array}$ & $\begin{array}{c}20-3 \\
109-111\end{array}$ & $\begin{array}{c}20-3 \\
109-111\end{array}$ & $\begin{array}{l}20-4 \\
13-16\end{array}$ & $\begin{array}{l}20-4 \\
13-16\end{array}$ & $\begin{array}{l}20-5 \\
3-10\end{array}$ & $\begin{array}{l}20-5 \\
71-75\end{array}$ & $\begin{array}{r}20-6 \\
27-34\end{array}$ & $\begin{array}{r}20-6 \\
44-53\end{array}$ & $\begin{array}{c}21-1 \text {, } \\
13-16\end{array}$ & $\begin{array}{l}22-1 \\
8-13\end{array}$ & $\begin{array}{l}22-1 \\
88-92\end{array}$ & $\begin{array}{l}22-2 \\
7-13\end{array}$ \\
\hline 236 & 237 & 238 & 239 & 239 & 239 & 240 & 241 & 242 & 243 & 244 & 245 & 246 & 247 \\
\hline \multirow[t]{2}{*}{ B } & B & B & B & B & B & B & B & B & B & B & B & B & B \\
\hline & & Glass & & Glass & Rock & & & & & & & & \\
\hline 48.12 & 49.70 & 48.78 & 48.58 & 49.61 & 49.40 & 49.34 & 48.60 & 49.15 & 48.22 & 47.96 & 47.95 & 48.02 & 48.44 \\
\hline 1.18 & 1.15 & 1.06 & 1.10 & 1.04 & 1.09 & 1.10 & 1.01 & 0.98 & 0.94 & 1.00 & 0.93 & 0.96 & 0.93 \\
\hline 17.70 & 17.78 & 17.13 & 18.10 & 17.57 & 16.87 & 16.88 & 18.24 & 17.55 & 17.21 & 18.54 & 17.65 & 17.34 & 17.57 \\
\hline 3.61 & 3.16 & 1.16 & 3.42 & 1.16 & 3.20 & 4.44 & 3.64 & 2.24 & 2.96 & 2.98 & 2.94 & 3.26 & 2.27 \\
\hline 5.05 & 5.30 & 6.94 & 5.16 & 6.82 & 5.16 & 4.16 & 4.35 & 5.49 & 4.64 & 4.85 & 4.54 & 4.62 & 5.14 \\
\hline 0.17 & 0.19 & 0.14 & 0.17 & 0.13 & 0.18 & 0.19 & 0.18 & 0.17 & 0.17 & 0.15 & 0.16 & 0.19 & 0.16 \\
\hline 6.09 & 6.74 & 7.52 & 6.77 & 7.71 & 7.07 & 7.15 & 6.97 & 7.97 & 7.90 & 6.97 & 7.68 & 7.80 & 7.42 \\
\hline 12.25 & 12.22 & 11.71 & 12.62 & 11.80 & 12.11 & 11.97 & 12.01 & 12.26 & 11.80 & 11.99 & 11.87 & 11.89 & 12.17 \\
\hline 2.58 & 2.63 & 2.48 & 2.48 & 2.58 & 2.68 & 2.63 & 2.64 & 2.57 & 2.58 & 2.48 & 2.64 & 2.50 & 2.57 \\
\hline 0.16 & 0.17 & 0.20 & 0.13 & 0.19 & 0.18 & 0.27 & 0.12 & 0.13 & 0.16 & 0.14 & 0.14 & 0.12 & 0.11 \\
\hline 0.09 & 0.11 & 0.06 & 0.06 & 0.08 & 0.10 & 0.09 & 0.09 & 0.07 & 0.07 & 0.06 & 0.07 & 0.09 & 0.06 \\
\hline 0.02 & 0.05 & 0.08 & 0.03 & 0109 & 0.02 & 0.02 & 0.04 & 0.08 & 0.01 & 0.04 & 0.01 & 0.04 & 0.08 \\
\hline 2.21 & 1.79 & 1.71 & 1.59 & 1.35 & 1.79 & 1.90 & 2.23 & 1.93 & 2.29 & 2.67 & 2.77 & 2.81 & 1.70 \\
\hline 99.24 & 100.99 & 98.97 & 100.21 & 100.13 & 99.84 & 100.15 & 100.12 & 100.59 & 98.95 & 100.83 & 99.36 & 99.64 & 98.62 \\
\hline 8.30 & 8.14 & 7.98 & 8.24 & 7.86 & 8.04 & 8.16 & 7.63 & 7.51 & 7.30 & 7.53 & 7.19 & 7.55 & 7.18 \\
\hline 0.592 & 0.621 & 0.651 & 0.619 & 0.660 & 0.635 & 0.634 & 0.644 & 0.677 & 0.682 & 0.647 & 0.679 & 0.672 & 0.672 \\
\hline 0.417 & 0.374 & 0.143 & 0.399 & 0.145 & 0.383 & 0.516 & 0.456 & 0.290 & 0.389 & 0.381 & 0.393 & 0.414 & 0.306 \\
\hline 1.2 & 1.7 & 1.8 & 0.8 & 2.8 & 3.1 & 5.6 & 0.7 & 1.4 & 2.7 & 1.0 & 2.2 & 1.5 & 1.5 \\
\hline 128 & 128 & 123 & 124 & 124 & 122 & 121 & 119 & 119 & 118 & 122 & 111 & 112 & 123 \\
\hline 25.4 & 24.6 & 21.9 & 22,4 & 23.9 & 22.5 & 24.2 & 22.2 & 24.5 & 20.1 & 20.7 & 20.1 & 21.1 & 19.7 \\
\hline 73 & 72 & 65 & 65 & 66 & 63 & 68 & 61 & 68 & 57 & 58 & 55 & 59 & 56 \\
\hline 1.4 & 1.6 & 0.5 & 1.3 & 1.0 & 1.5 & 0.7 & 1.0 & 1.0 & 1.0 & 0.7 & 1.8 & 0.6 & 1.4 \\
\hline
\end{tabular}

However, these average compositions still reflect minor effects of compositional changes produced by basalt-seawater interaction. Table 4 is a listing of 89 microprobe analyses of natural glasses that occur in basalts at both Sites 395 and 396. The same notations and conventions that apply to the bulk rock data in Tables 1 and 2 are used in Table 4. Sample numbers in the JSC series from Site 396 are equivalent to the same numbers used in Rhodes et al. (1978). In addition to microprobe analyses of natural glasses, three-element reconnaissance microprobe analyses of olivine and plagioclase phenocrysts and microphenocrysts are presented for a representative suite of rock samples. Table 5 is a representative listing of spinel analyses (six elements) from phyric basalts in Holes 396 and 396B.

In addition, we have performed one-atmosphere melting experiments on three natural rock powders to determine their melting relations. The compositions of mineral phases coexisting with liquid in these experimental charges have been determined as a function of temperature (Table 6). Linear cooling-rate experiments have been performed on all three rocks at three cooling rates to determine the dependence of textural variation on cooling history.

Our purpose is to relate the mineralogy and mineral chemistry of the various flow units to the bulk chemistry and the crystallization histories of the individual samples. Integration of these data with the results of experimental melting studies provides some constraints on the fractionation of the units and their interrelationships. This combination of techniques has led us to the conclusion that the mixing of magmas is an important factor in the differentiation of the Hole 396B basalts.

\section{ANALYTICAL TECHNIQUES}

The major element data were obtained by X-ray fluorescence analysis (XRF) of fused glass discs, prepared by fusing the sample with a lanthanum-bearing lithium borate fusion mixture (Norrish and Hutton, 1969). $\mathrm{FeO}$ was determined titrimetrically using the modified cold acid digestion method of Wilson (Maxwell, 1968); $\mathrm{Fe}_{2} \mathrm{O}_{3}$ was obtained by difference from the XRF total iron value. $\mathrm{Na}_{2} \mathrm{O}$ was obtained on a separate 20 to $30 \mathrm{mg}$ aliquot by instrumental neutron activation analysis (INAA). Total water content was measured coulometrically using a DuPont moisture analyzer, in which the sample is fused with a lead oxide flux.

The trace elements $(\mathrm{Rb}, \mathrm{Sr}, \mathrm{Y}, \mathrm{Zr}, \mathrm{Nb}$, and $\mathrm{Ni}$ ) were determined by XRF analysis on pressed powder pellets. Corrections were made for non-linear background, tube contamination, and inter-element interferences (Norrish and Chappell, 1967). Corrections for matrix effects were based on a modification of the Compton scattering method (Reynolds, 1967), using the Ag-Compton peak. Additional trace element data (Table 2; $\mathrm{La}, \mathrm{Ce}, \mathrm{Sm}, \mathrm{Eu}, \mathrm{Tb}, \mathrm{Yb}, \mathrm{Lu}$, $\mathrm{Hf}, \mathrm{Cr}, \mathrm{Sc}$, and $\mathrm{Ni}$ ) were obtained by INAA following the methods of Jacobs et al. (in press). 
TABLE 2

Trace Element Abundances (ppm) in Selected Samples From Holes 396 and 396B

\begin{tabular}{|c|c|c|c|c|c|c|c|c|c|c|c|c|c|c|}
\hline Hole & 396 & 396 & 396 & 396 & $396 \mathrm{~B}$ & $396 \mathrm{~B}$ & $396 \mathrm{~B}$ & $396 \mathrm{~B}$ & 396B & $396 \mathrm{~B}$ & $396 \mathrm{~B}$ & $396 \mathrm{~B}$ & $396 \mathrm{~B}$ & $396 B$ \\
\hline $\begin{array}{c}\text { Sample } \\
\text { (Interval in } \mathrm{cm} \text { ) }\end{array}$ & $\begin{array}{l}13-1 \\
73-76\end{array}$ & $\begin{array}{l}\text { 15-1, } \\
110-115\end{array}$ & $\begin{array}{l}23-1 \\
58-61\end{array}$ & $\stackrel{25-1}{134-139}$ & $\begin{array}{c}4-1 \\
119-122\end{array}$ & $\begin{array}{c}7-1 \\
50-57\end{array}$ & $\begin{array}{c}7 \cdot 2 \\
118-122\end{array}$ & $\begin{array}{r}8-1 \\
76-82\end{array}$ & $\begin{array}{r}9-2 \\
74-82\end{array}$ & $\begin{array}{l}10-2 \\
14-18\end{array}$ & $\begin{array}{c}13-1 \\
102-105\end{array}$ & $\begin{array}{l}13 \cdot 3 \\
3-5\end{array}$ & $\begin{array}{l}14-1, \\
9-13\end{array}$ & $\begin{array}{c}15 \cdot 2 \\
120-133\end{array}$ \\
\hline Depth $(\mathrm{m})$ & 109 & 128 & 202 & 221 & 154 & 176 & 182 & 185 & 199 & 209 & 221 & 225 & 226 & 238 \\
\hline Unit & - & - & - & - & $A_{1}$ & $A_{1}$ & $A_{1}$ & $\mathrm{~A}_{2}$ & $\mathrm{~A}_{2}$ & $\mathrm{~A}_{2}$ & $\mathrm{~A}_{2}$ & $\mathrm{~A}_{3}$ & $\mathrm{~A}_{3}$ & $\mathrm{~A}_{3}$ \\
\hline JSC No. & 177 & 173 & 172 & 170 & 180 & 185 (Std) & 189 & 190 & 192 & 195 & 203 & 207 & 208 & 214 (Std) \\
\hline Sc & 38.8 & 33.4 & 34.5 & 35.0 & 38.6 & 38.2 & 38.7 & 38.7 & 38.6 & 38.7 & 39.1 & 38.8 & 39.5 & 38.6 \\
\hline $\mathrm{Cr}$ & 310 & 440 & 300 & 290 & 350 & 340 & 300 & 310 & 300 & 300 & 300 & 290 & 300 & 300 \\
\hline Ni & 115 & 175 & 90 & 70 & 140 & 150 & 80 & 100 & 110 & 95 & 120 & 100 & 110 & 130 \\
\hline La & 3.64 & 2.72 & 2.54 & 2.75 & 2.99 & 2.88 & 3.1 & 3.21 & 3.21 & 3.34 & 3.16 & 3.75 & 3.76 & 3.65 \\
\hline $\mathrm{Ce}$ & 11.5 & 8.9 & 8.4 & 8.9 & 9.9 & 10.0 & 10.6 & 10.4 & 10.7 & 10.7 & 10.8 & 11.5 & 12.0 & 12.5 \\
\hline $\mathrm{Sm}$ & 3.85 & 2.82 & 2.99 & 3.02 & 3.25 & 3.25 & 3.51 & 3.48 & 3.44 & 3.62 & 3.52 & 3.94 & 4.06 & 3.96 \\
\hline Eu & 1.38 & 1.04 & 1.11 & 1.12 & 1.18 & 1.18 & 1.29 & 1.24 & 1.25 & 1.26 & 1.27 & 1.44 & 1.43 & 1.41 \\
\hline $\mathrm{Tb}$ & 1.0 & 0.71 & 0.78 & 0.85 & 0.84 & 0.84 & 0.88 & 0.89 & 0.94 & 0.93 & 0.93 & 0.96 & 0.96 & 1.0 \\
\hline $\mathrm{Yb}$ & 3.5 & 2.48 & 2.79 & 3.0 & 3.2 & 3.2 & 3.2 & 3.2 & 3.3 & 3.6 & 3.5 & 3.6 & 3.7 & 3.8 \\
\hline $\mathrm{Lu}$ & 0.54 & 0.39 & 0.44 & 0.45 & 0.45 & 0.49 & 0.50 & 0.52 & 0.52 & 0.53 & 0.52 & 0.57 & 0.61 & 0.55 \\
\hline $\mathrm{Hf}$ & 3.1 & 2.3 & 2.4 & 2.4 & 2.5 & 2.4 & 3.1 & 2.8 & 2.7 & 2.7 & 2.7 & 2.7 & 3.1 & 3.1 \\
\hline
\end{tabular}
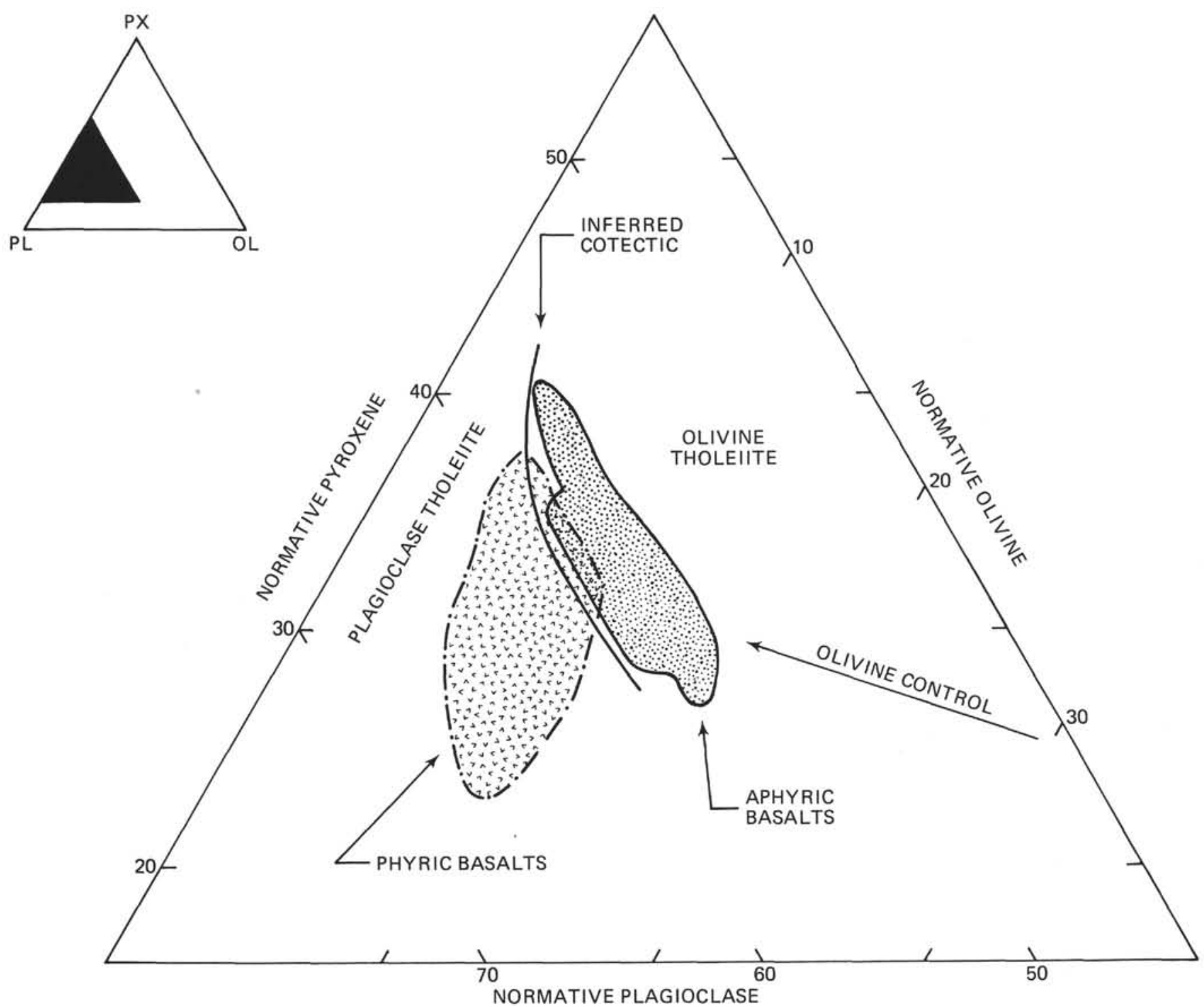

Figure 1. Normative olivine-plagioclase-pyroxene relationships in basalts from both Legs 45 and 46 . The empirically determined olivine-plagioclase cotectic is from Shido et al. (1971). 
TABLE 2 - Continued

\begin{tabular}{|c|c|c|c|c|c|c|c|c|c|c|c|c|c|}
\hline $396 \mathrm{~B}$ & $396 \mathrm{~B}$ & $396 \mathrm{~B}$ & $396 \mathrm{~B}$ & $396 \mathrm{~B}$ & $396 \mathrm{~B}$ & $396 \mathrm{~B}$ & $396 \mathrm{~B}$ & $396 \mathrm{~B}$ & $396 \mathrm{~B}$ & $396 \mathrm{~B}$ & $396 \mathrm{~B}$ & $396 \mathrm{~B}$ & $396 \mathrm{~B}$ \\
\hline $\begin{array}{r}15-4 \\
85-90\end{array}$ & $\begin{array}{r}16-3 \\
46-51\end{array}$ & $\begin{array}{c}16-5 \\
130-134\end{array}$ & $\begin{array}{l}17-4, \\
75-81\end{array}$ & $\begin{array}{l}18-1 \\
|24-13|\end{array}$ & $\begin{array}{c}20-1 \\
136-138\end{array}$ & $\begin{array}{c}20-3 \\
109-111\end{array}$ & $\begin{array}{l}21-2 \\
13-16\end{array}$ & $\begin{array}{r}22-3 \\
28-38\end{array}$ & $\begin{array}{l}23-1 \text {, } \\
46-50\end{array}$ & $\begin{array}{l}24-1, \\
94-98\end{array}$ & $\begin{array}{c}30-1 \text {, } \\
77-80\end{array}$ & $\begin{array}{c}32-1 . \\
30-34\end{array}$ & $\begin{array}{r}32-1, \\
34-36\end{array}$ \\
\hline 242 & 249 & 254 & 273 & 275 & 288 & 291 & 297 & 311 & 318 & 333 & 382 & 389 & 389 \\
\hline$A_{3}$ & B & B & B & B & B & B & B & B & C & C & C & C & C \\
\hline 220 & 226 & 227 & 230 & 232 & 236 & 238 & 244 & 249 (Std) & 252 & 255 & 257 & 259 & 260 \\
\hline 39.9 & 35.2 & 34.3 & 33.5 & 33.2 & 33.2 & 32.6 & 32.1 & 31.7 & 38.6 & 37.4 & 36.0 & 36.5 & 37.0 \\
\hline 295 & 320 & 330 & 315 & 320 & 340 & 350 & 340 & 370 & 320 & 330 & 310 & 320 & 310 \\
\hline 140 & 100 & 120 & 120 & 90 & 120 & 120 & 150 & 110 & 130 & 130 & 110 & 100 & 110 \\
\hline 3.77 & 2.53 & 2.44 & 2.31 & 2.43 & 2.30 & 1.95 & 2.07 & 1.89 & 4.39 & 4.19 & 3.97 & 3.97 & 4.10 \\
\hline 11.4 & 8.0 & 8.5 & 8.2 & 7.9 & 8.0 & 6.9 & 6.6 & 6.4 & 13.0 & 13.2 & 12.0 & 12.0 & 12.5 \\
\hline 4.0 & 2.87 & 2.83 & 2.71 & 2.68 & 2.69 & 2.42 & 2.31 & 2.33 & 3.81 & 3.55 & 3.62 & 3.62 & 3.56 \\
\hline 1.45 & 1.08 & 1.04 & 1.06 & 1.05 & 0.99 & 0.93 & 0.99 & 0.89 & 1.28 & 1.26 & 1.26 & 1.26 & 1.30 \\
\hline 0.97 & 0.79 & 0.78 & 0.69 & 0.78 & 0.65 & 0.63 & 0.64 & 0.60 & 1.0 & 0.86 & 0.89 & 0.89 & 0.94 \\
\hline 3.7 & 2.9 & 2.7 & 2.5 & 2.7 & 2.5 & 2.4 & 2.2 & 2.2 & 3.6 & 3.3 & 3.4 & 3.4 & 3.4 \\
\hline 0.58 & 0.41 & 0.43 & 0.39 & 0.40 & 0.43 & 0.40 & 0.34 & 0.36 & 0.53 & 0.53 & 0.50 & 0.50 & 0.55 \\
\hline 3.5 & 2.2 & 2.1 & 2.5 & 2.1 & 1.9 & 1.8 & 1.8 & 1.6 & 3.0 & 2.7 & 2.6 & 2.8 & 3.0 \\
\hline
\end{tabular}

TABLE 3

Average Compositions of Chemically Defined Basalt Types From Hole 396B

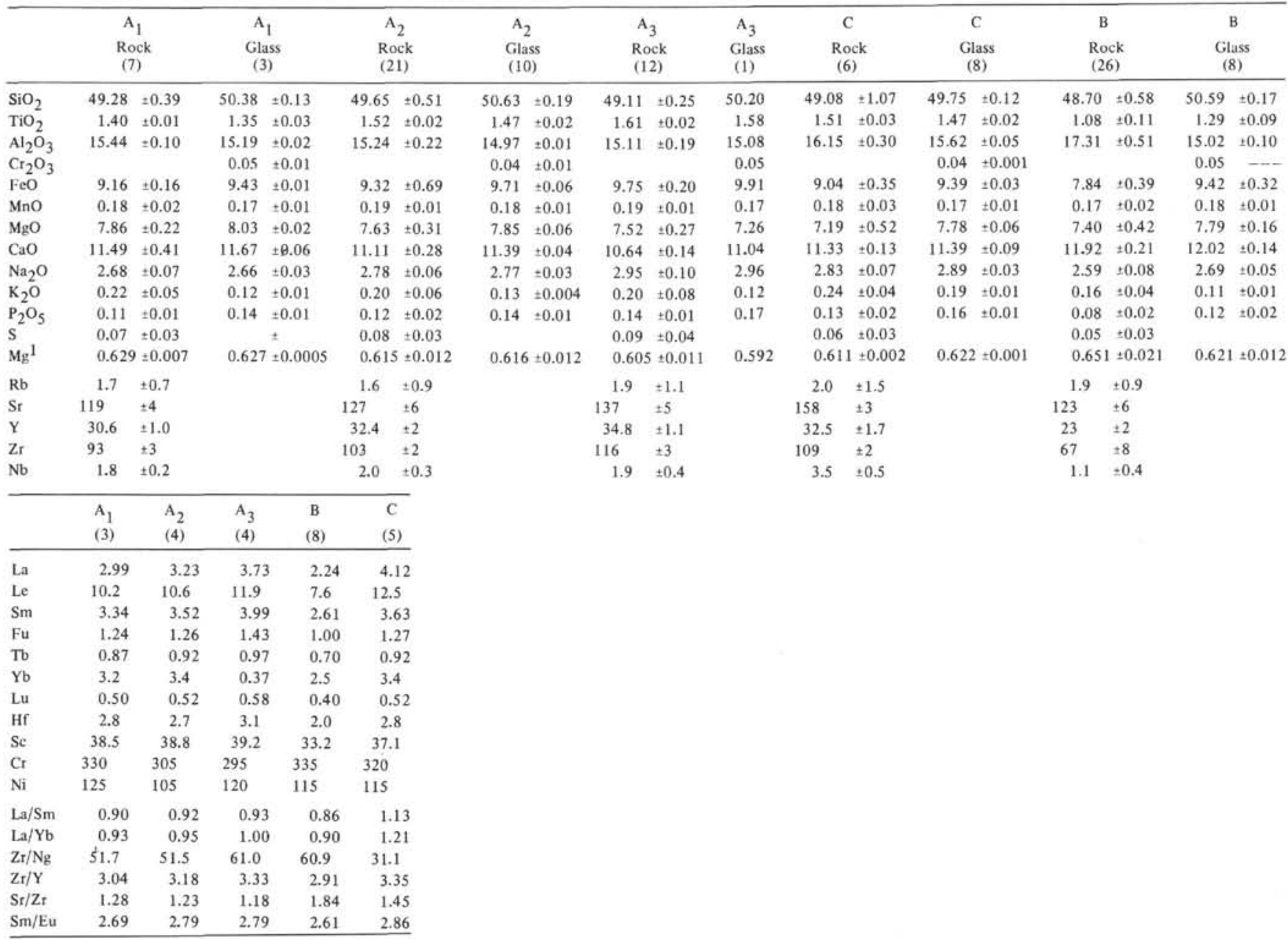

Microprobe analyses were performed on a three-channel ARL-EMX instrument at standard operating conditions using natural mineral standards.

\section{BASALT CHEMISTRY}

All the basalts recovered from Holes 396 and 396B are olivine and hypersthene-normative and plot in the olivine tholeiite field of the basalt tetrahedron (Yoder and Tilley, 1962). Alkali basalts and transitional tholeiites are conspicuously absent, as are quartz tholeiites. They have the overall compositional characteristics typical of mid-ocean ridge tholeiites, as is evidenced by their relatively constant $\mathrm{SiO}_{2}$ concentrations and low $\mathrm{TiO}_{2}$ abundances, together with low total alkali content and 
TABLE 4

Electron Microprobe Analyses of Residual Glasses and Glass Inclusions in Olivine and Plagioclase

\begin{tabular}{|c|c|c|c|c|c|c|c|c|c|c|c|c|c|c|}
\hline \multicolumn{15}{|c|}{ Hole 396B } \\
\hline $\begin{array}{c}\text { Sample } \\
\text { (Interval in } \mathrm{cm} \text { ) }\end{array}$ & $\begin{array}{c}4-1 \\
119-122\end{array}$ & $\begin{array}{c}7-1 \\
10-14\end{array}$ & $\begin{array}{c}7-2 \\
16-20\end{array}$ & $\begin{array}{c}8-1 \\
76-82\end{array}$ & $\begin{array}{c}9-2, \\
74-82\end{array}$ & $\begin{array}{c}\text { 10-1, } \\
96-101\end{array}$ & $\begin{array}{l}10-2, \\
29-33\end{array}$ & $\begin{array}{c}11-1, \\
70-73\end{array}$ & $\begin{array}{c}11-1, \\
78-86\end{array}$ & $\begin{array}{c}13-1 \\
102-105\end{array}$ & $\begin{array}{r}13-2, \\
21-25\end{array}$ & $\begin{array}{c}13-2, \\
130-134\end{array}$ & $\begin{array}{c}13-2 \\
137-140\end{array}$ & $\begin{array}{l}14-1, \\
9-13\end{array}$ \\
\hline Unit & $\mathrm{A}_{1}$ & $\mathrm{~A}_{1}$ & $A_{1}$ & $\mathrm{~A}_{2}$ & $\mathrm{~A}_{2}$ & $\mathrm{~A}_{2}$ & $\mathrm{~A}_{2}$ & $\mathrm{~A}_{2}$ & $\mathrm{~A}_{2}$ & $\mathrm{~A}_{2}$ & $\mathrm{~A}_{2}$ & $\mathrm{~A}_{2}$ & $\mathrm{~A}_{2}$ & $\mathrm{~A}_{3}$ \\
\hline JSC No. & 180 & 183 & 187 & 190 & 192 & 194 & 195 & 197 & 198 & 202 & 203 & 205 & 206 & 208 \\
\hline $\mathrm{SiO}_{2}$ & 50.39 & 50.24 & 50.50 & 50.55 & 50.43 & 50.49 & 50.94 & 50.77 & 50.68 & 50.60 & 50.76 & 50.76 & 50.32 & 50.20 \\
\hline $\mathrm{TiO}_{2}$ & 1.34 & 1.38 & 1.33 & 1.45 & 1.47 & 1.47 & 1.47 & 1.43 & 1.47 & 1.48 & 1.48 & 1.49 & 1.45 & 1.58 \\
\hline $\mathrm{Al}_{2} \mathrm{O}_{3}$ & 15.20 & 15.17 & 15.21 & 14.99 & 14.96 & 14.96 & 14.98 & 14.97 & 14.97 & 14.97 & 14.98 & 15.00 & 14.95 & 15.08 \\
\hline $\mathrm{Cr}_{2} \mathrm{O}_{3}$ & 0.04 & 0.06 & 0.04 & 0.05 & 0.02 & 0.04 & 0.04 & 0.05 & 0.04 & 0.04 & 0.03 & 0.04 & 0.05 & 0.05 \\
\hline $\mathrm{FeO}$ & 9.44 & 9.42 & 9.44 & 9.70 & 9.75 & 9.78 & 9.67 & 9.71 & 9.80 & 9.67 & 9.72 & 9.74 & 9.59 & 9.91 \\
\hline $\mathrm{MnO}$ & 0.18 & 0.18 & 0.16 & 0.17 & 0.18 & 0.18 & 0.19 & 0.18 & 0.18 & 0.16 & 0.17 & 0.18 & 0.19 & 0.17 \\
\hline $\mathrm{MgO}$ & 8.02 & 8.06 & 8.02 & 7.95 & 7.82 & 7.89 & 7.84 & 7.87 & 7.88 & 7.76 & 7.84 & 7.88 & 7.78 & 7.26 \\
\hline $\mathrm{CaO}$ & 11.74 & 11.62 & 11.66 & 11.41 & 11.43 & 11.40 & 11.40 & 11.42 & 11.35 & 11.41 & 11.42 & 11.38 & 11.31 & 11.04 \\
\hline $\mathrm{Na}_{2} \mathrm{O}$ & 2.65 & 2.70 & 2.64 & 2.78 & 2.78 & 2.78 & 2.79 & 2.78 & 2.80 & 2.77 & 2.75 & 2.77 & 2.70 & 2.96 \\
\hline $\mathrm{K}_{2} \mathrm{O}$ & 0.12 & 0.12 & 0.11 & 0.13 & 0.12 & 0.13 & 0.13 & 0.13 & 0.13 & 0.13 & 0.13 & 0.13 & 0.12 & 0.12 \\
\hline $\mathrm{P}_{2} \mathrm{O}_{5}$ & 0.13 & 0.14 & 0.14 & 0.14 & 0.15 & 0.16 & 0.12 & 0.13 & 0.13 & 0.15 & 0.14 & 0.15 & 0.16 & 0.17 \\
\hline Total & 99.25 & 99.09 & 99.25 & 99.32 & 99.09 & 99.28 & 99.57 & 99.44 & 99.43 & 99.11 & 99.42 & 99.52 & 98.62 & 98.44 \\
\hline Mg' & 0.627 & 0.628 & 0.627 & 0.619 & 0.614 & 0.615 & 0.616 & 0.616 & 0.614 & 0.614 & 0.615 & 0.616 & 0.616 & 0.592 \\
\hline$q$ & - & - & - & - & - & - & - & - & - & - & - & - & - & - \\
\hline or & $0.71^{\mathrm{a}}$ & - & - & $0.77^{\mathrm{a}}$ & - & - & - & - & - & - & - & - & - & 0.71 \\
\hline ab & 22.51 & - & - & 23.52 & - & - & - & - & - & - & - & - & - & 25.05 \\
\hline an & 29.15 & - & - & 27.99 & - & - & - & - & - & - & - & - & - & 27.51 \\
\hline di & 22.82 & - & - & 22.61 & - & - & - & - & - & - & - & - & - & 21.50 \\
\hline hy & 14.36 & - & - & 15.10 & - & - & - & - & - & - & - & - & - & 13.86 \\
\hline ol & 5.30 & - & - & 4.69 & - & - & - & - & - & - & - & - & - & 4.98 \\
\hline mgt & 1.52 & - & - & 1.57 & - & - & - & - & - & - & - & - & - & 1.59 \\
\hline chr & 0.06 & - & - & 0.06 & - & - & - & - & - & - & - & - & - & 0.07 \\
\hline il & 2.56 & - & - & 2.79 & - & - & - & - & - & - & - & - & - & 3.00 \\
\hline ap & 0.28 & - & - & 0.33 & - & - & - & - & - & - & - & - & - & 9.37 \\
\hline phase & res. & res. & res. & res. & res. & res. & res. & res. & res. & res. & res. & res. & res. & res. \\
\hline
\end{tabular}

TABLE 4 - Continued

\begin{tabular}{|c|c|c|c|c|c|c|c|c|c|c|c|c|c|c|}
\hline \multirow[b]{2}{*}{$\begin{array}{c}\text { Sample } \\
\text { (Interval in } \mathrm{cm} \text { ) }\end{array}$} & \multicolumn{2}{|c|}{ Hole $396 \mathrm{~B}$} & \multirow{2}{*}{$\begin{array}{c}\text { Hole } 396 \\
15-1 \\
110-115\end{array}$} & \multirow{2}{*}{$\begin{array}{c}\text { Hole } 395 \\
\begin{array}{c}18-2 \\
43-46\end{array}\end{array}$} & \multirow[b]{2}{*}{$\begin{array}{r}17-1 \\
46-55\end{array}$} & \multirow[b]{2}{*}{$\begin{array}{c}26-2 \\
125-129\end{array}$} & \multirow[b]{2}{*}{$\begin{array}{c}28-1, \\
116-122\end{array}$} & \multirow[b]{2}{*}{$\begin{array}{r}56-3 \\
62-65\end{array}$} & \multirow[b]{2}{*}{$\begin{array}{r}17-1 \\
46-55\end{array}$} & \multirow[b]{2}{*}{$\begin{array}{c}26-2 \\
125-129\end{array}$} & \multirow[b]{2}{*}{$\begin{array}{c}26-2 \\
125-129\end{array}$} & \multirow[b]{2}{*}{$\begin{array}{l}29-1 \\
125-131\end{array}$} & \multicolumn{2}{|c|}{ Hole $395 \mathrm{~A}$} \\
\hline & $\begin{array}{r}30-1, \\
77-80\end{array}$ & $\begin{array}{l}32-1 \\
34-36\end{array}$ & & & & & & & & & & & $\begin{array}{c}28-1 \\
116-122\end{array}$ & $\begin{array}{c}28-1 \\
116-122\end{array}$ \\
\hline Unit & C & $\mathrm{C}$ & B & $P_{1}$ & $\mathrm{P}_{3}$ & $\mathrm{P}_{4}$ & $\mathrm{P}_{5}$ & $A_{3}$ & $\mathrm{P}_{3}$ & $\mathrm{P}_{4}$ & $\mathrm{P}_{4}$ & $P_{5}$ & $\mathrm{P}_{5}$ & $\mathrm{P}_{5}$ \\
\hline JSC No. & 257 & 260 & 173 & 115 & 131 & 137 & 140 & 154 & $131-1$ & $137-1$ & $137-2$ & $141-1$ & $140-1$ & $140-2$ \\
\hline $\mathrm{SiO}_{2}$ & 49.65 & 49.87 & 50.23 & 50.69 & 51.03 & 51.07 & 50.65 & 50.44 & 51.69 & 51.35 & 51.28 & 51.51 & 50.78 & 50.57 \\
\hline $\mathrm{TiO}_{2}$ & 1.48 & 1.49 & 1.34 & 1.44 & 1.38 & 1.47 & 1.58 & 1.73 & 1.34 & 1.18 & 1.15 & 1.08 & 1.48 & 1.57 \\
\hline $\mathrm{Al}_{2} \mathrm{O}_{3}$ & 15.55 & 15.65 & 15.64 & 14.23 & 14.49 & 14.34 & 14.75 & 14.93 & 17.45 & 15.11 & 15.21 & 17.69 & 14.29 & 14.03 \\
\hline $\mathrm{Cr}_{2} \mathrm{O}_{3}$ & 0.04 & 0.05 & 0.05 & 0.04 & 0.06 & 0.04 & 0.04 & 0.03 & 0.05 & 0.04 & 0.04 & 0.05 & 0.05 & 0.05 \\
\hline $\mathrm{FeO}$ & 9.39 & 9.44 & 9.06 & 9.98 & 9.06 & 9.98 & 9.88 & 9.90 & 7.58 & 9.61 & 9.67 & 7.62 & 10.13 & 10.09 \\
\hline $\mathrm{MnO}$ & 0.16 & 0.19 & 0.18 & 0.14 & 0.16 & 0.17 & 0.20 & 0.21 & 0.13 & 0.15 & 0.16 & 0.16 & 0.17 & 0.18 \\
\hline $\mathrm{MgO}$ & 7.72 & 7.73 & 7.94 & 7.42 & 7.63 & 7.28 & 7.24 & 7.51 & 4.35 & 6.89 & 6.27 & 3.59 & 7.50 & 7.50 \\
\hline $\mathrm{CaO}$ & 11.40 & 11.50 & 11.32 & 11.77 & 11.96 & 11.58 & 11.50 & 10.79 & 13.20 & 12.37 & 12.92 & 14.78 & 11.50 & 11.48 \\
\hline $\mathrm{Na}_{2} \mathrm{O}$ & 2.86 & 2.88 & 2.83 & 2.59 & 2.51 & 2.83 & 3.07 & 2,93 & 3.02 & 2.60 & 2.56 & 2.73 & 3.01 & 3.01 \\
\hline $\mathrm{K}_{2} \mathrm{O}$ & 0.18 & 0.18 & 0.13 & 0.11 & 0.11 & 0.12 & 0.12 & 0.15 & 0.12 & 0.10 & 0.09 & 0.06 & 0.14 & 0.14 \\
\hline $\mathrm{P}_{2} \mathrm{O}_{5}$ & 0.15 & 0.15 & - & - & - & - & - & - & - & - & - & - & 0.11 & 0.15 \\
\hline Total & 98.58 & 99.17 & 98.73 & 98.41 & 99.21 & 98.80 & 99.40 & 99.02 & 98.93 & 99.40 & 99.33 & 99.27 & 99.16 & 98.77 \\
\hline $\mathrm{Mg}^{\prime}$ & 0.620 & 0.619 & 0.634 & 0.600 & 0.605 & 0.593 & 0.583 & 0.591 & 0.532 & 0.587 & 0.592 & 0.483 & 0.594 & 0.595 \\
\hline$q$ & - & - & - & - & - & - & - & - & 1.29 & - & 0.27 & 1.75 & - & - \\
\hline or & - & - & 0.77 & 0.65 & 0.65 & 0.71 & 0.71 & 0.89 & 0.71 & 0.59 & 0.59 & 0.35 & 0.83 & 0.83 \\
\hline$a b$ & - & - & 23.95 & 21.92 & 21.24 & 23.95 & 25.98 & 24.79 & 25.56 & 22.00 & 21.66 & 23.10 & 25.47 & 25.47 \\
\hline an & - & - & 29.59 & 26.88 & 27.95 & 26.07 & 26.11 & 27.14 & 33.71 & 29.26 & 29.72 & 35.84 & 25.07 & 24.36 \\
\hline di & - & - & 21.65 & 25.80 & 25.68 & 25.70 & 25.37 & 21.61 & 26.21 & 26.34 & 28.31 & 31.17 & 25.66 & 25.96 \\
\hline hy & - & - & 11.70 & 17.83 & 18.67 & 15.86 & 9.34 & 14.33 & 7.71 & 17.31 & 15.12 & 3.79 & 11.37 & 11.43 \\
\hline ol & - & - & 7.09 & 1.04 & 0.84 & 2.18 & 7.30 & 5.39 & - & 0.15 & - & - & 6.12 & 5.83 \\
\hline mgt & - & - & 1.45 & 1.61 & 1.59 & 1.59 & 1.65 & 1.65 & 1.22 & 1.55 & 1.55 & 1.23 & 1.64 & 1.62 \\
\hline chr & - & - & 0.07 & 0.06 & 0.09 & 0.06 & 0.04 & 0.07 & 0.07 & 0.06 & 0.06 & 0.07 & 0.07 & 0.07 \\
\hline il & - & - & 2.54 & 2.73 & 2.62 & 2.79 & 3.00 & 3.29 & 2.54 & 2.24 & 2.18 & 2.05 & 2.81 & 2.98 \\
\hline ap & - & - & - & - & - & - & - & - & - & - & - & - & 0.24 & 0.33 \\
\hline phase & res. & res. & res. & res. & res. & res. & res. & res. & oliv. & oliv. & oliv. & oliv. & plag. & plag. \\
\hline
\end{tabular}


TABLE 4 - Continued

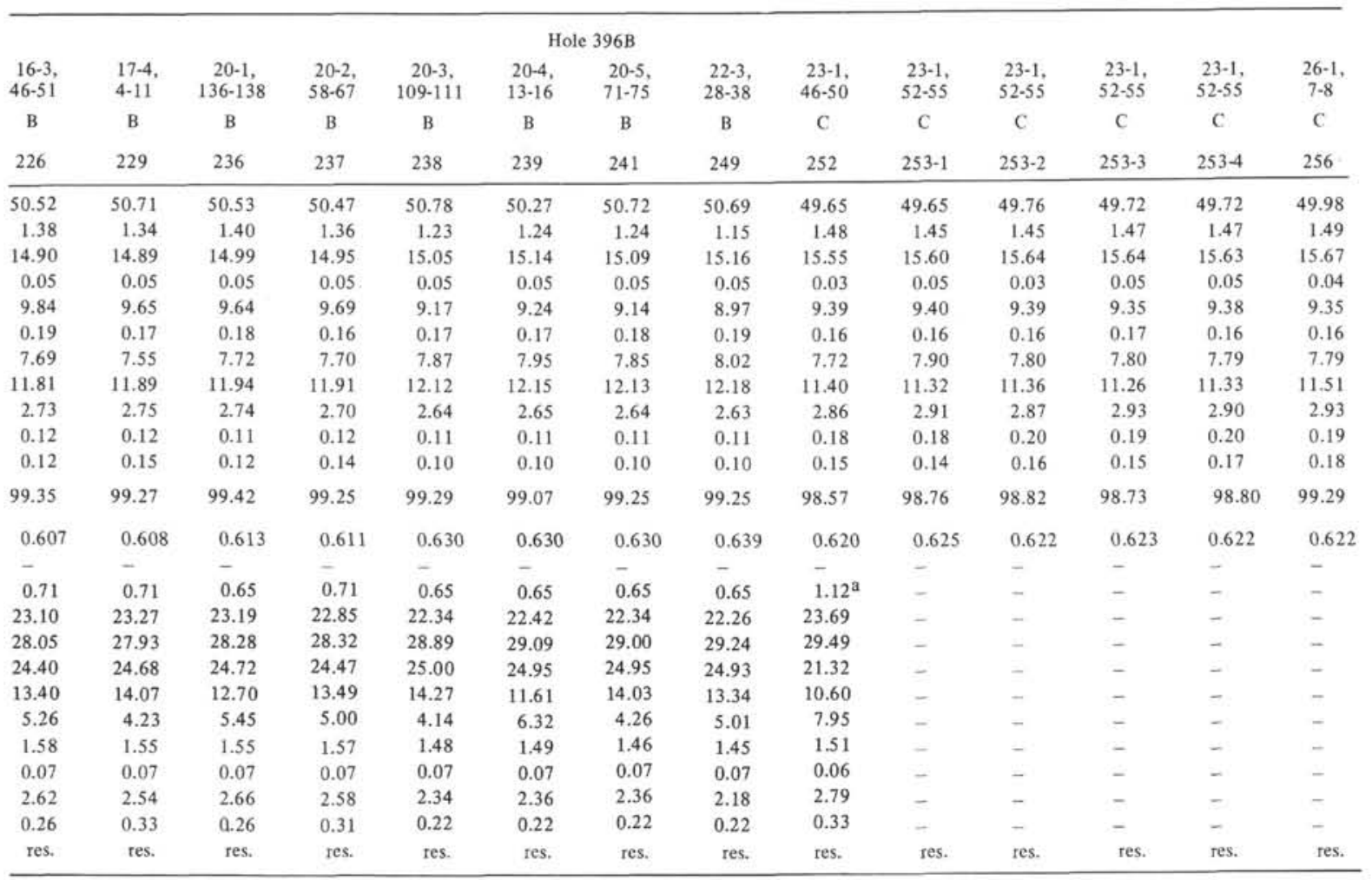

TABLE 4 - Continued

\begin{tabular}{|c|c|c|c|c|c|c|c|c|c|c|c|c|c|}
\hline \multirow[b]{2}{*}{$\begin{array}{c}28-1, \\
116-122\end{array}$} & \multicolumn{3}{|c|}{ Hole 395 A } & \multirow[b]{2}{*}{$\begin{array}{c}28-1 \\
116-122\end{array}$} & \multirow[b]{2}{*}{$\begin{array}{c}28-1, \\
116-122\end{array}$} & \multirow[b]{2}{*}{$\begin{array}{c}28-1 \\
116-122\end{array}$} & \multirow[b]{2}{*}{$\begin{array}{c}28-1 \\
116-122\end{array}$} & \multicolumn{6}{|c|}{ Hole 395} \\
\hline & $\begin{array}{c}28-1 \\
116-122\end{array}$ & $\begin{array}{c}28-1, \\
116-122\end{array}$ & $\begin{array}{c}28-1, \\
116-122\end{array}$ & & & & & $\begin{array}{r}18-2 \\
43-46\end{array}$ & $\begin{array}{r}18-2 \\
43-46\end{array}$ & $\begin{array}{r}18-2 \\
43-46\end{array}$ & $\begin{array}{c}18-2 \\
43-46\end{array}$ & $\begin{array}{r}18-2 \\
43-46\end{array}$ & $\begin{array}{r}18-2 \\
43-46\end{array}$ \\
\hline $\mathrm{P}_{5}$ & $\mathrm{P}_{5}$ & $\mathrm{P}_{5}$ & $\mathrm{P}_{5}$ & $\mathrm{P}_{5}$ & $P_{5}$ & $\mathrm{P}_{5}$ & $\mathrm{P}_{5}$ & $P_{1}$ & $P_{1}$ & $P_{1}$ & $\mathrm{P}_{1}$ & $P_{1}$ & $\mathrm{P}_{1}$ \\
\hline $140-3$ & $140-4$ & $140-5$ & $140-6$ & $140-7$ & $140-8$ & $140-9$ & $140-10$ & $115-1$ & $115-2$ & $115-3$ & $115-4$ & $115-5$ & $115-6$ \\
\hline 50.64 & 50.42 & 50.46 & 50.89 & 50.74 & 51.05 & 50.43 & 50.61 & 49.61 & 49.56 & 50.63 & 51.70 & 51.06 & 50.81 \\
\hline 1.60 & 1.51 & 1.57 & 1.06 & 1.15 & 1.37 & 1.23 & 1.18 & 0.86 & 0.85 & 1.47 & 1.46 & 1.08 & 1.16 \\
\hline 14.63 & 15.34 & 16.00 & 14.62 & 14.67 & 15.10 & 14.87 & 16.62 & 14.28 & 14.19 & 14.42 & 17.13 & 14.51 & 14.64 \\
\hline 0.05 & 0.04 & 0.05 & 0.04 & 0.05 & 0.04 & 0.03 & 0.04 & 0.06 & 0.06 & 0.03 & 0.03 & 0.06 & 0.06 \\
\hline 9.93 & 9.83 & 9.72 & 9.77 & 10.10 & 9.70 & 10.03 & 7.61 & 9.39 & 9.27 & 10.31 & 7.85 & 8.99 & 9.40 \\
\hline 0.17 & 0.18 & 0.17 & 0.18 & 0.18 & 0.19 & 0.18 & 0.20 & 0.13 & 0.14 & 0.16 & 0.12 & 0.15 & 0.18 \\
\hline 7.43 & 6.28 & 6.04 & 7.68 & 7.71 & 7.14 & 7.95 & 7.29 & 10.00 & 10.00 & 6.31 & 4.00 & 8.24 & 8.52 \\
\hline 11.51 & 12.27 & 12.97 & 11.48 & 11.53 & 11.48 & 11.30 & 12.64 & 11.89 & 11.96 & 12.53 & 12.69 & 12.42 & 11.77 \\
\hline 3.04 & 2.92 & 2.89 & 3.02 & 2.96 & 3.15 & 2.96 & 2.73 & 2.21 & 2.21 & 2.57 & 2.81 & 2.56 & 2.58 \\
\hline 0.16 & 0.13 & 0.11 & 0.14 & 0.14 & 0.11 & 0.13 & 0.10 & 0.07 & 0.07 & 0.11 & 0.12 & 0.11 & 0.10 \\
\hline 0.14 & 0.14 & 0.14 & 0.07 & 0.09 & - & - & - & - & - & - & - & 0.09 & 0.09 \\
\hline 99.20 & 99.06 & 100.12 & 98.95 & 99.32 & 99.33 & 99.11 & 99.02 & 98.50 & 98.31 & 98.54 & 97.91 & 99.27 & 99.32 \\
\hline 0.597 & 0.558 & 0.552 & 0.609 & 0.602 & 0.593 & 0.611 & 0.655 & 0.678 & 0.681 & 0.548 & 0.502 & 0.645 & 0.642 \\
\hline- & - & - & - & - & - & - & - & - & - & 0.15 & 3.46 & - & - \\
\hline 0.95 & 0.77 & 0.65 & 0.83 & 0.83 & 0.65 & 0.77 & 0.59 & 0.41 & 0.41 & 0.65 & 0.71 & 0.65 & 0.59 \\
\hline 25.72 & 24.71 & 24.46 & 25.56 & 25.05 & 26.66 & 25.05 & 23.10 & 18.70 & 18.70 & 21.75 & 23.78 & 21.66 & 21.83 \\
\hline 25.80 & 28.37 & 30.36 & 25.92 & 26.33 & 26.74 & 26.91 & 31.16 & 28.84 & 28.59 & 27.49 & 33.77 & 27.78 & 28.07 \\
\hline 24.94 & 26.06 & 27.30 & 25.06 & 24.86 & 24.76 & 23.85 & 25.63 & 24.46 & 24.94 & 28.58 & 24.15 & 27.12 & 24.27 \\
\hline 10.37 & 10.20 & 7.29 & 10.42 & 10.46 & 9.83 & 9.45 & 10.59 & 13.69 & 13.31 & 15.55 & 8.04 & 14.61 & 15.89 \\
\hline 6.62 & 4.16 & 5.25 & 7.46 & 7.83 & 6.59 & 9.22 & 3.90 & 9.27 & 9.26 & - & - & 3.76 & 4.76 \\
\hline 1.59 & 1.58 & 1.57 & 1.58 & 1.62 & 1.57 & 1.61 & 1.23 & 1.51 & 1.49 & 1.67 & 1.26 & 1.45 & 1.51 \\
\hline 0.07 & 0.06 & 0.07 & 0.06 & 0.07 & 0.06 & 0.04 & 0.06 & 0.09 & 0.09 & 0.04 & 0.04 & 0.09 & 0.09 \\
\hline 3.04 & 2.87 & 2.98 & 2.01 & 2.18 & 2.60 & 2.34 & 2.24 & 1.63 & 1.61 & 2.79 & 2.77 & 2.05 & 2.20 \\
\hline 0.31 & 0.31 & 0.31 & 0.15 & 0.20 & - & - & - & - & - & - & - & 0.20 & 0.20 \\
\hline oliv. & oliv. & plag. & plag. & plag. & oliv. & plag. & oliv. & plag. & plag. & oliv. & oliv. & plag. & plag. \\
\hline
\end{tabular}


M. A. DUNGAN ET AL.

TABLE 4 - Continued

\begin{tabular}{|c|c|c|c|c|c|c|c|c|c|c|c|c|c|c|}
\hline \multirow[b]{2}{*}{$\begin{array}{c}\text { Sample } \\
\text { (Interval in cm) }\end{array}$} & \multicolumn{6}{|c|}{ Hole 395} & \multicolumn{2}{|c|}{ Hole $395 \mathrm{~A}$} & \multicolumn{6}{|c|}{ Hole 396B } \\
\hline & $\begin{array}{r}18-2 \\
43-46\end{array}$ & $\begin{array}{r}18-2 \\
43-46\end{array}$ & $\begin{array}{r}18-2 \\
43-46\end{array}$ & $\begin{array}{r}18-2 \\
43-46\end{array}$ & $\begin{array}{r}18-2 \\
43-46\end{array}$ & $\begin{array}{r}18-2 \\
43-46\end{array}$ & $\begin{array}{r}18-2 \\
49-51\end{array}$ & $\begin{array}{r}18-2 \\
49-51\end{array}$ & $\begin{array}{r}16-3 \\
46-51\end{array}$ & $\begin{array}{r}16-3 \\
46-51\end{array}$ & $\begin{array}{r}16-3 \\
46-51\end{array}$ & $\begin{array}{l}16-3 \\
46-5 i\end{array}$ & $\begin{array}{c}20-1 \\
136-138\end{array}$ & $\begin{array}{l}20-1 \\
136-138\end{array}$ \\
\hline Unit & $\mathrm{P}_{1}$ & $\mathrm{P}_{1}$ & $P_{1}$ & $P_{1}$ & $P_{1}$ & $P_{1}$ & $P_{1}$ & $P_{1}$ & B & B & B & B & B & B \\
\hline JSC No. & $115-7$ & $115-8$ & $115-9$ & $115-10$ & $115-11$ & $115-12$ & $45 \mathrm{G}-1$ & $45 G-2$ & $226-1$ & $226-2$ & $226-3$ & $226-4$ & $236-1$ & $236 \cdot 2$ \\
\hline $\mathrm{SiO}_{2}$ & 50.58 & 52.24 & 52.13 & 52.17 & 52.23 & 52.45 & 51.22 & 51.89 & 50.43 & 50.38 & 50.07 & 50.81 & 50.54 & 50.51 \\
\hline $\mathrm{TiO}_{2}$ & 1.21 & 0.80 & 0.82 & 0.84 & 0.80 & 0.84 & 0.77 & 1.01 & 1.06 & 1.23 & 0.81 & 1.16 & 1.11 & 1.01 \\
\hline $\mathrm{Al}_{2} \mathrm{O}_{3}$ & 14.58 & 13.88 & 14.11 & 13.88 & 13.93 & 13.99 & 16.98 & 16.50 & 16.12 & 14.78 & 17.79 & 15.88 & 15.45 & 16.54 \\
\hline $\mathrm{Cr}_{2} \mathrm{O}_{3}$ & 0.05 & 0.05 & 0.05 & 0.04 & 0.05 & 0.06 & 0.07 & 0.07 & 0.05 & 0.06 & 0.05 & 0.05 & 0.06 & 0.05 \\
\hline $\mathrm{FeO}$ & 9.39 & 8.61 & 8.49 & 8.69 & 8.27 & 8.40 & 7.80 & 7.75 & 8.21 & 10.04 & 6.67 & 9.18 & 9.39 & 7.92 \\
\hline $\mathrm{MnO}$ & 0.14 & 0.16 & 0.17 & 0.14 & 0.13 & 0.15 & 0.15 & 0.15 & 0.14 & 0.11 & 0.10 & 0.16 & 0.18 & 0.14 \\
\hline $\mathrm{MgO}$ & 8.46 & 8.79 & 8.66 & 8.80 & 8.70 & 8.66 & 5.33 & 4.73 & 6.72 & 7.73 & 7.91 & 7.67 & 6.83 & 7.61 \\
\hline $\mathrm{CaO}$ & 11.39 & 12.18 & 12.12 & 12.29 & 12.18 & 12.26 & 15.11 & 15.09 & 13.54 & 11.68 & 12.28 & 11.83 & 13.26 & 13.00 \\
\hline $\mathrm{Na}_{2} \mathrm{O}$ & 2.04 & 2.36 & 2.36 & 2.38 & 2.38 & 2.39 & 2.00 & 2.20 & 2.51 & 2.92 & 2.35 & 2.80 & 2.45 & 2.49 \\
\hline $\mathrm{K}_{2} \mathrm{O}$ & 0.11 & 0.09 & 0.09 & 0.09 & 0.09 & 0.09 & 0.04 & 0.06 & 0.10 & 0.14 & 0.06 & 0.12 & 0.09 & 0.11 \\
\hline $\mathrm{P}_{2} \mathrm{O}_{5}$ & 0.07 & 0.05 & 0.05 & 0.05 & 0.06 & 0.06 & 0.05 & 0.07 & 0.11 & 0.09 & 0.06 & 0.08 & 0.10 & 0.09 \\
\hline Total & 98.02 & 99.21 & 99.05 & 99.37 & 98.82 & 99.35 & 99.52 & 99.52 & 98.99 & 99.16 & 99.15 & 99.74 & 99.46 & 99.47 \\
\hline $\mathrm{Mg}^{\prime}$ & 0.641 & 0.669 & 0.670 & 0.667 & 0.676 & 0.671 & 0.575 & 0.547 & 0.618 & 0.604 & 0.701 & 0.623 & 0.590 & 0.656 \\
\hline$q$ & 1.16 & 0.61 & 0.71 & 0.30 & 0.89 & 0.92 & 2.26 & 3.33 & - & - & - & - & - & - \\
\hline or & 0.65 & 0.53 & 0.63 & 0.53 & 0.53 & 0.53 & 0.24 & 0.35 & 0.59 & 0.83 & 0.35 & 0.71 & 0.53 & 0.65 \\
\hline$a b$ & 17.26 & 19.97 & 19.97 & 20.14 & 20.14 & 20.22 & 16.92 & 18.62 & 21.24 & 24.71 & 19.89 & 23.69 & 20.73 & 21.07 \\
\hline an & 30.30 & 27.02 & 27.64 & 26.93 & 27.06 & 27.18 & 37.24 & 34.97 & 32.42 & 26.81 & 37.82 & 30.41 & 30.90 & 33.63 \\
\hline di & 21.01 & 26.91 & 26.16 & 27.43 & 26.79 & 27.03 & 30.79 & 32.56 & 27.86 & 25.05 & 22.40 & 22.71 & 28.13 & 24.66 \\
\hline hy & 23.71 & 21.18 & 21.02 & 20.98 & 20.44 & 20.39 & 9.23 & 6.36 & 10.41 & 9.13 & 10.36 & 12.26 & 21.84 & 11.33 \\
\hline ol & - & - & - & - & - & - & - & - & 2.91 & 8.51 & 5.59 & 6.14 & 2.43 & 4.76 \\
\hline $\mathrm{mgt}$ & 1.51 & 1.39 & 1.36 & 1.41 & 1.33 & 1.35 & 1.26 & 1.25 & 1.32 & 1.62 & 1.07 & 1.48 & 1.51 & 1.28 \\
\hline chr & 0.07 & 0.07 & 0.07 & 0.06 & 0.07 & 0.09 & 0.10 & 0.10 & 0.07 & 0.09 & 0.07 & 0.07 & 0.09 & 0.07 \\
\hline il & 2.30 & 1.52 & 1.56 & 1.60 & 1.52 & 1.60 & 1.46 & 1.92 & 2.01 & 2.34 & 1.54 & 2.20 & 2.11 & 1.92 \\
\hline ap & 0.15 & 0.11 & 0.11 & 0.11 & 0.13 & 0.13 & 0.11 & 0.15 & 0.24 & 0.20 & 0.13 & 0.17 & 0.22 & 0.20 \\
\hline phase & plag. & plag. & plag. & plag. & plag. & plag. & oliv. & oliv. & oliv. & oliv. & oliv. & oliv. & oliv. & oliv. \\
\hline
\end{tabular}

TABLE 4 - Continued

\begin{tabular}{|c|c|c|c|c|c|}
\hline \multirow[b]{2}{*}{$\begin{array}{c}\text { Sample } \\
\text { (Interval in } \mathrm{cm} \text { ) }\end{array}$} & \multicolumn{3}{|c|}{ Hole 396B } & \multirow[b]{2}{*}{$\begin{array}{l}22-3 \\
28-38\end{array}$} & \multirow[b]{2}{*}{$\begin{array}{r}22-3 \\
28-38\end{array}$} \\
\hline & $\begin{array}{l}20-4, \\
13-16\end{array}$ & $\begin{array}{l}20-5 \\
71-75\end{array}$ & $\begin{array}{r}22-3, \\
28-38\end{array}$ & & \\
\hline Unit & B & B & B & B & B \\
\hline JSC No. & $239-8$ & $241-1$ & $249-1$ & $249-2$ & $249-3$ \\
\hline $\mathrm{SiO}_{2}$ & 50.58 & 51.67 & $50 ? 81$ & 50.50 & 50.41 \\
\hline $\mathrm{TiO}_{2}$ & 1.13 & 1.30 & 0.91 & 1.16 & 1.16 \\
\hline $\mathrm{Al}_{2} \mathrm{O}_{3}$ & 15.29 & 19.80 & 16.30 & 16.54 & 16.19 \\
\hline $\mathrm{Cr}_{2} \mathrm{O}_{3}$ & 0.05 & 0.06 & 0.06 & 0.06 & 0.05 \\
\hline $\mathrm{FeO}$ & 9.46 & 5.92 & 7.49 & 8.26 & 8.25 \\
\hline $\mathrm{MnO}$ & 0.17 & 0.14 & 0.15 & 0.14 & 0.15 \\
\hline $\mathrm{MgO}$ & 8.29 & 2.71 & 7.96 & 5.34 & 5.87 \\
\hline $\mathrm{CaO}$ & 11.91 & 15.71 & 13.33 & 14.27 & 14.26 \\
\hline $\mathrm{Na}_{2} \mathrm{O}$ & 2.74 & 2.65 & 2.42 & 2.60 & 2.58 \\
\hline $\mathrm{K}_{2} \mathrm{O}$ & 0.12 & 0.11 & 0.10 & 0.11 & 0.11 \\
\hline $\mathrm{P}_{2} \mathrm{O}_{5}$ & 0.09 & 0.12 & 0.09 & 0.11 & 0.09 \\
\hline Total & 99.83 & 100.19 & 99.62 & 99.09 & 99.12 \\
\hline Mg' & 0.634 & 0.476 & 0.680 & 0.561 & 0.585 \\
\hline$q$ & - & 2.76 & - & - & - \\
\hline or & 0.71 & 0.65 & 0.59 & 0.65 & 0.65 \\
\hline$a b$ & 23.19 & 22.42 & 20.48 & 22.00 & 21.83 \\
\hline an & 29.07 & 41.81 & 33.32 & 33.14 & 32.27 \\
\hline di & 24.05 & 28.29 & 26.18 & 30.45 & 31.12 \\
\hline hy & 10.70 & - & 11.64 & 7.98 & 6.80 \\
\hline ol & 8.27 & - & 4.28 & 1.10 & 2.74 \\
\hline mgt & 1.52 & 0.96 & 1.20 & 1.33 & 1.33 \\
\hline chr & 0.07 & 0.09 & 0.09 & 0.09 & 0.07 \\
\hline il & 2.15 & 2.47 & 1.73 & 2.20 & 2.20 \\
\hline ap & 0.20 & 0.26 & 0.20 & 0.24 & 0.20 \\
\hline phase & plag. & oliv. & oliv. & oliv. & oliv. \\
\hline
\end{tabular}


TABLE 4 - Continued

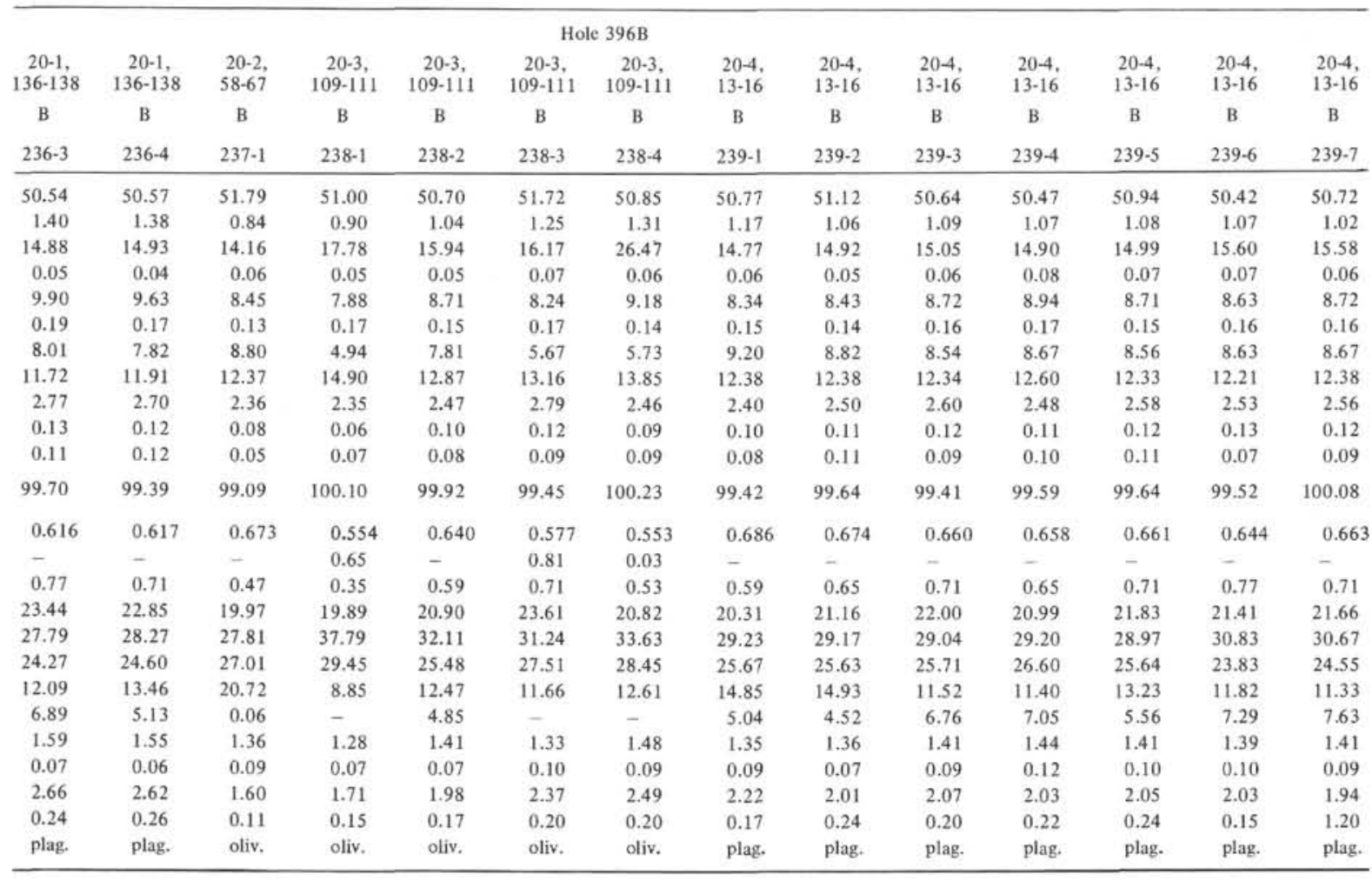

$\mathrm{K}_{2} \mathrm{O} / \mathrm{Na}_{2} \mathrm{O}$ ratios (e.g., Engel et al., 1965; Melson et al., 1976). $\mathrm{K}_{2} \mathrm{O}$ abundances are characteristically low in the majority of samples $(<0.2 \%)$ and, where comparative data are available, do not differ significantly from the $\mathrm{K}_{2} \mathrm{O}$ content of basaltic glass from the same samples. A few samples have higher $\mathrm{K}_{2} \mathrm{O}$ contents not related to basalt type, presumably the result of seawater alteration, although this is not always correlated with total water content or the oxidation ratio.

The majority of samples selected for analysis were relatively fresh, with total water contents usually less than 2 per cent and with oxidation ratios mostly below 0.4 . Consequently, it was hoped that the major or trace element chemistry of these samples would not have been significantly modified by seawater alteration. The mobile elements $\mathrm{K}, \mathrm{Rb}$, and $\mathrm{S}$ are notable exceptions. The effects of alteration of sulfur are particularly pronounced, and there is a marked inverse correlation between the oxidation ratio and the sulfur content. It would appear from this relationship that unaltered samples should contain about 0.14 per cent sulfur, a value somewhat greater than that proposed by Moore and Fabbi (1971) as typical for unaltered basalts recovered from deep water. Many of the samples, particularly those which are obviously altered, also have lost substantial amounts of $\mathrm{MgO}$.

Two broad basalt types, differing fundamentally in both petrography and whole-rock chemistry, are prevalent at Site 396 . These are, respectively, the aphyric (sparsely phyric of the Site Report, this volume) and phyric basalts. The aphyric basalts contain less than 1 per cent megascopic phenocrysts, but contain microphenocrysts of olivine and plagioclase, whereas the phyric basalts contain abundant (10 to $30 \%$ ) plagioclase phenocrysts, together with phenocrysts of olivine and minor chromian spinel.

Chemically, the phyric basalts are distinguished from the aphyric basalts by higher $\mathrm{Al}_{2} \mathrm{O}_{3}$ (16.4 to $18.7 \%$ ) and $\mathrm{CaO}$ (11.5 to $12.3 \%$ ) concentrations, and by lower $\mathrm{SiO}_{2}, \mathrm{FeO}$, and $\mathrm{MgO}$ concentrations. These differences are reflected in higher normative plagioclase contents and in lower $\mathrm{Ab} /(\mathrm{Ab}+\mathrm{An})$ ratios in the phyric basalts. Although both total iron and $\mathrm{MgO}$ are lower in the phyric basalts; $\mathrm{Mg}^{\prime}$ values tend to be higher, an indication that they are more "primitive" than the aphyric basalts (Figures 2 and 3). $\mathrm{TiO}_{2}$, and most other minor and magmaphile trace element abundances are markedly lower in the phyric basalts (Figure 2). Strontium, on the other hand, is higher in the phyric basalts, both in absolute terms and with respect to other magmaphile elements. Consequently, $\mathrm{Sr} / \mathrm{Zr}$ ratios are distinctly different in the two basalt types, varying somewhat more in the phyric basalts (1.7 to 2.2 ) in contrast to the lower values ( 1.15 to 1.30 and 1.5 to 1.9 ) found in the two aphyric units (Table 3 ).

Figure 1 illustrates the normative olivine, plagioclase, and pyroxene relationships for these basalts, relative to the inferred olivine-plagioclase cotectic of Shido et al. (1971). All the aphyric basalts plot in the olivine tholeiite field, 
TABLE 5

Microprobe Analyses of Chromium Spinel in Phyric Basalts

\begin{tabular}{|c|c|c|c|c|c|c|c|c|c|}
\hline \multirow[b]{2}{*}{ Unit } & 1 & 2 & 3 & 4 & 5 & 6 & 7 & 8 & 9 \\
\hline & $\mathrm{A}_{3}$ & B & B & B & B & B & D & B & B \\
\hline JSC No. & 209 & 235 & 235 & 226 & 226 & 226 & 758 & 258 & 259 \\
\hline $\mathrm{TiO}_{2}$ & 0.34 & 0.72 & 0.42 & 0.64 & 0.33 & 1.00 & 0.92 & 0.69 & 1.05 \\
\hline $\mathrm{Al}_{2} \mathrm{O}_{3}$ & 35.9 & 24.7 & 32.5 & 24.8 & 35.8 & 24.4 & 23.2 & 27.3 & 25.8 \\
\hline $\mathrm{Cr}_{2} \mathrm{O}_{3}$ & 27.4 & 38.7 & 31.7 & 36.9 & 24.1 & 36.1 & 38.8 & 34.7 & 34.8 \\
\hline $\mathrm{Fe}_{2} \mathrm{O}_{3} *$ & 6.4 & 7.3 & 6.6 & 8.0 & 5.5 & 8.2 & 8.3 & 8.3 & 7.9 \\
\hline $\mathrm{FeO}$ & 12.7 & 13.3 & 11.5 & 14.4 & 10.7 & 15.2 & 13.4 & 13.1 & 13.8 \\
\hline $\mathrm{MnO}$ & 0.18 & 0.25 & 0.21 & 0.29 & 0.17 & 0.29 & 0.26 & 0.23 & 0.25 \\
\hline $\mathrm{MgO}$ & 16.2 & 15.0 & 16.8 & 14.0 & 17.5 & 13.6 & 14.8 & 15.3 & 14.6 \\
\hline Total & 99.1 & 100.0 & 99.7 & 99.0 & 99.1 & 98.8 & 99.7 & 99.6 & 98.2 \\
\hline $\mathrm{Ti}$ & 0.060 & 0.134 & 0.075 & 0.121 & 0.058 & 0.190 & 0.173 & 0.127 & 0.198 \\
\hline $\mathrm{Cr}$ & 9.946 & 7.180 & 9.051 & 7.324 & 9.816 & 7.255 & 6.828 & 7.881 & 7.609 \\
\hline $\mathrm{Al}$ & 5.090 & 7.544 & 5.920 & 7.307 & 5.351 & 7.198 & 7.658 & 6.717 & 6.882 \\
\hline $\mathrm{Fe}^{+3}$ & 1.105 & 1.318 & 1.152 & 1.470 & 0.943 & 1.520 & 1.521 & 1.495 & 1.452 \\
\hline $\mathrm{Fe}^{+2}$ & 2.448 & 2.68 & 2.224 & 2.951 & 2.047 & 3.134 & 2.739 & 2.625 & 2.822 \\
\hline Mn & 0.036 & 0.052 & 0.042 & 0.062 & 0.033 & 0.062 & 0.055 & 0.048 & 0.053 \\
\hline Ms & 5.674 & 5.512 & 5.915 & 5.226 & 6.066 & 5.112 & 5.507 & 5.583 & 5.443 \\
\hline Total & 24.422 & 24.505 & 24.440 & 24.564 & 24.359 & 24.584 & 24.584 & 24.574 & 24.557 \\
\hline Total & 3.6161 & 4.083 & 3.438 & 4.525 & 3.034 & 4.767 & 4.364 & 4.218 & 4.373 \\
\hline $\mathrm{Cr} / \mathrm{Cr}+\mathrm{Al}$ & 0.339 & 0.512 & 0.395 & 0.499 & 0.353 & 0.498 & 0.529 & 0.460 & 0.475 \\
\hline $\mathrm{Mg} / \mathrm{Mg}+\mathrm{Fe}$ & 0.611 & 0.574 & 0.632 & 0.536 & 0.667 & 0.517 & 0.588 & 0.570 & 0.554 \\
\hline
\end{tabular}

Note: ${ }^{*} \mathrm{Fe}_{2} \mathrm{O}_{3}$ calculated on the assumption of stoichiometry. JSC Rock numbers correspond to DSDP sample numbers listed in Table 1. (1) Spinel included in the average of a plagioclase in a glomorophyric aggregate; $(2,3)$ Two grains included in the same plagioclase phenocryst; $(4,5)$ Spinel grains included in olivine and plagioclase phenocrysts, respectively, from the same rock; (6) Groundmass spinel (web-shaped) from same rock as 4 and $5 ;(7,9)$ Groundmass spinel from Unit D basalt; (8) Spinel grain in olivine phenocryst.

TABLE 6

Compositions of Olivine and Plagioclase From Microprobe Analyses in Melting Experiments on Three Rocks From Hole 396B

\begin{tabular}{cccccccc}
\hline $\begin{array}{c}\text { Temperature } \\
\left({ }^{\circ} \mathrm{C}\right)\end{array}$ & $\begin{array}{c}\text { Fo Content } \\
\text { of Olivine }\end{array}$ & $\begin{array}{c}\text { An Content of } \\
\text { Plagioclase }\end{array}$ & $\begin{array}{c}\text { Sample } 7-1,42-46 \mathrm{~cm} \\
\text { Fo Content } \\
\text { of Olivine }\end{array}$ & $\begin{array}{c}\text { An Content of } \\
\text { Plagioclase }\end{array}$ & $\begin{array}{c}\text { Fo Content } \\
\text { of Olivine }\end{array}$ & $\begin{array}{c}\text { An Content of } \\
\text { Plagioclase }\end{array}$ \\
\hline 1225 & & & & & $81.4 \pm 0.3(2)$ & $87.0 \pm 0.1(8)$ & $83.8 \pm 0.9(4)$ \\
1200 & & & & & & & \\
1175 & $83.6 \pm 0.2(4)^{\mathrm{a}}$ & $74.3 \pm 0.6(6)$ & $84.0 \pm 0.3(5)$ & $73.1 \pm 0.8(9)$ & $86.0 \pm 0.8(7)$ & $77.5 \pm 1.8(4)$ \\
1150 & $81.2 \pm 0.45(5)$ & $67.2 \pm 1.4(5)$ & $79.7 \pm 1.7(5)$ & $65.5 \pm 0.5(6)$ & $78.0 \pm 0.2(6)$ & $67.4 \pm 0.8(4)$ \\
\hline
\end{tabular}

${ }^{\mathrm{a}}$ Error limits are 1 sigma based on the number of analyses shown in parentheses.

within a tightly controlled elongate group sub-parallel to the inferred cotectic. In contrast, the phyric basalts plot predominantly within the plagioclase tholeiite field and are scattered widely. Those phyric basalts plotting close to the inferred cotectic, or just within the olivine tholeiite field, have the lowest $\mathrm{Al}_{2} \mathrm{O}_{3}$ content. Presumably, they contain fewer plagioclase phenocrysts than the majority of samples, and may be closer to melt compositions.

Initial studies, based on shipboard X-ray fluorescence analyses, recognized seven compositionally distinct magmatic units and sub-units within the three major basalt types. These were, in order of increasing depth below the sediment-basement interface, A1, A2, A3, B1, B2, C, and D.

Our data essentially confirm these subdivisions, subject to minor revisions in the light of the more recent and comprehensive analyses. Average values, calculated from the data in Table 1 , are given in Table 3 . The only change we have made in the classificatory scheme is to combine the sub-units of B into a single unit because it is a chemically, magnetically, and stratigraphically coherent unit which exhibits substantial internal variation in composition. "Unit D' ' consists of several clasts of phyric basalt mixed with the more abundant Type $\mathrm{C}$ that comprises the lower clastic unit 


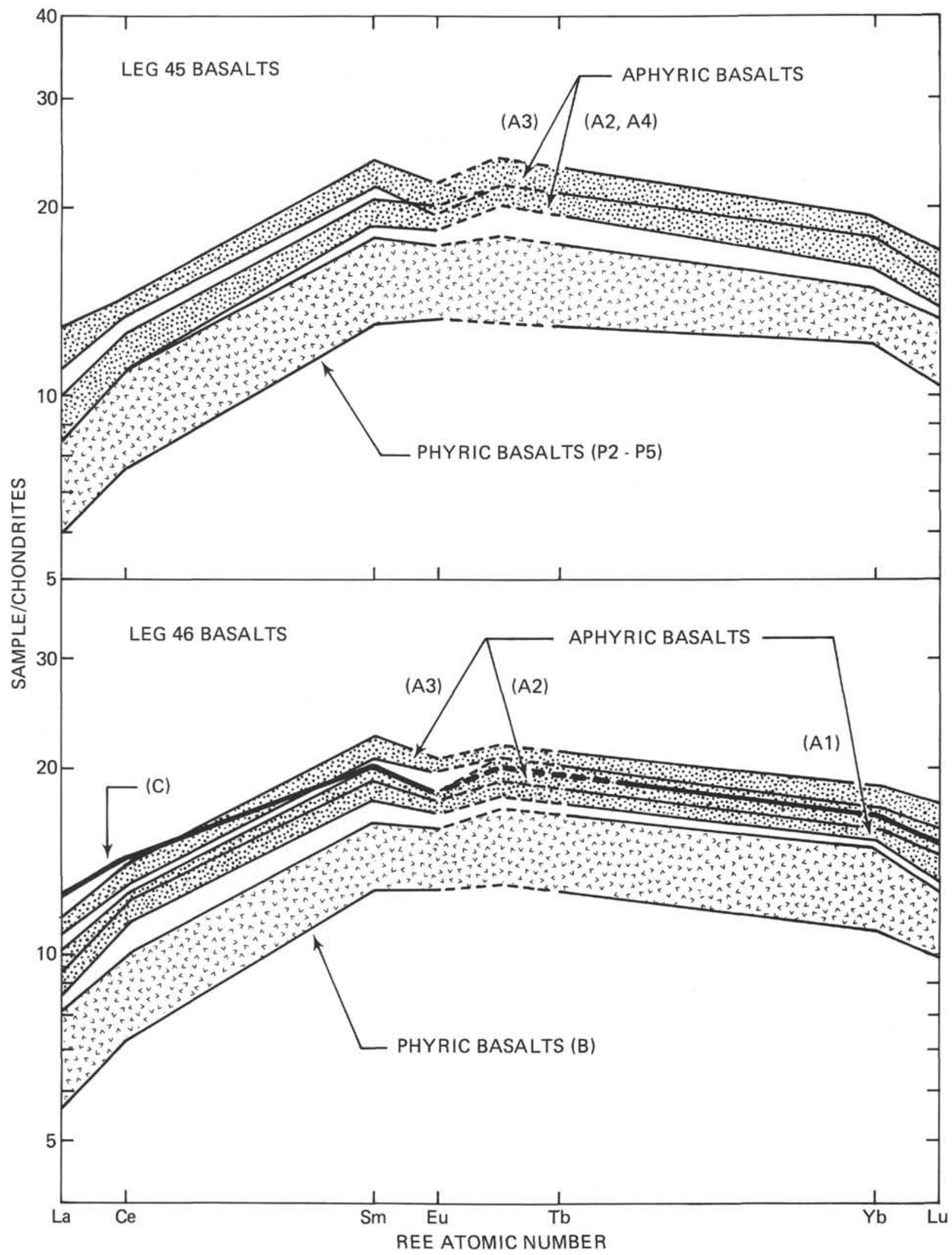

Figure 2. Chondrite-normalized REE abundances in basalts from Legs 45 and 46. Chemically defined units used in this paper and in Rhodes et al. (1978) are used here. Note the similarity in both total abundances and $\mathrm{La} / \mathrm{Sm}$ at the two sites. 


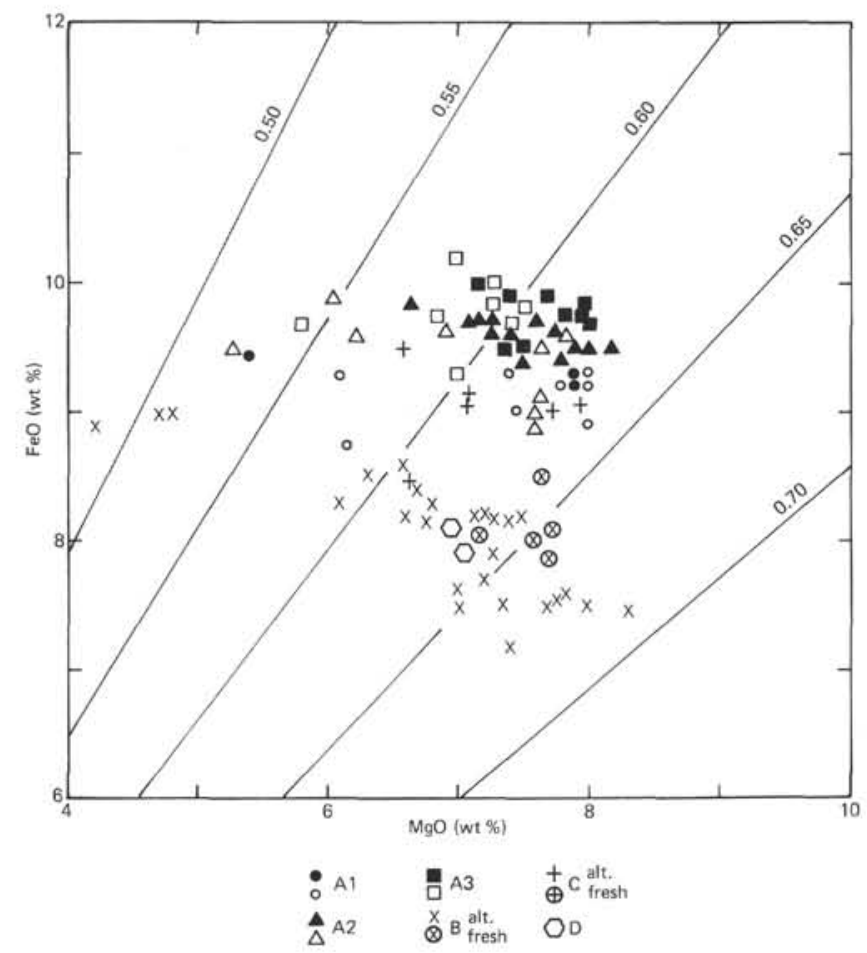

Figure 3. $\mathrm{FeO}-\mathrm{MgO}$ relationships in altered and fresh basalts of Hole 396B. Reference lines are for constant $\mathrm{Mg}^{\prime}$.

in Hole $396 \mathrm{~B}$ and is, therefore, not actually a stratigraphic unit in itself.

The minor and trace element abundances of the Site 396 basalts are within the range of typical mid-ocean ridge tholeiites (Kay et al., 1970; Schilling, 1971, 1975; Pearce and Cann, 1973; Hart, 1976; Bryan et al., 1976; Erlank and Kable, 1976). This is illustrated (Figure 2, Table 3) by the chondrite-normalized rare-earth patterns, which range between 13 and 24 times chondritic abundances for $\mathrm{Sm}$, and which display the light rare-earth depletion typical of "normal" or Type I ocean ridge basalts (Bryan et al., 1976). $\mathrm{Mg}^{\prime}$ values in these rocks are variable, ranging from about 0.59 to 0.69 (Figure 2), which is within the range characteristic of mid-ocean ridge basalts but is lower than the values found in the most primitive basalts identified to date (Frey et al., 1974; Bryan and Moore, 1976; Rhodes et al., 1978). These values, together with low $\mathrm{Ni}$ concentrations, moderately high magmaphile element abundances, and the presence of multiple phenocryst phases are taken as evidence that these basalts have undergone substantial differentiation.

The chondrite-normalized REE patterns of the basalts at Site 395 are also shown on Figure 2. Note that the abundances and degree of LREE-depletion are virtually identical at both sites with the exception of Unit C (Hole 396B) which has higher $\mathrm{La} / \mathrm{Sm}$ and other magmaphile element ratios that differ markedly from Units A and B and the Site 395 basalts. The remaining aphyric and phyric units exhibit small variations in trace element ratios which are, in terms of general trends, consistent with very small amounts of fractional crystallization. Figure 4 is an example of two elements, $\mathrm{Zr}$ and $\mathrm{Y}$, that vary systematically with increasing degree of fractionation within Units A and B. Note, however, that as the basalts become increasingly evolved, the $\mathrm{Zr} / \mathrm{Y}$ ratio increases slightly from 2.8 at the base of Unit $\mathrm{B}$ to 3.3 in $\mathrm{A}_{3}$. Other ratios change systematically as well; e.g., $\mathrm{La} / \mathrm{Sm}, \mathrm{La} / \mathrm{Yb}$, and $\mathrm{Sm} / \mathrm{Eu}$ increase whereas $\mathrm{Sr} / \mathrm{Zr}$ decreases. Rhodes et al. (1978) have modeled the possible relationships among several units at Site 395 and found that the degrees of magmaphile element-enrichment for different elements are not consistent in detail with a simple fractional crystallization scheme. An additional mechanism that will provide enrichment of the incompatible elements is required.

One of the most useful indicators of differentiation in basaltic rocks is the atomic ratio $\mathrm{Mg} /\left(\mathrm{Mg}+\mathrm{Fe}^{+2}\right)$, or $\mathrm{Mg}^{\prime}$, which is very sensitive to olivine fractionation; e.g., removal of 5 per cent olivine from a liquid with an $\mathrm{Mg}^{\prime}$ of 0.64 lowers the ratio to 0.56 . Unfortunately, the $\mathrm{Mg}^{\prime}$ of some of the basalts in Hole 396B has been modified by seawater alteration as $\mathrm{MgO}$ is lost during this process. Figure 3 is a plot of $\mathrm{MgO}$ versus $\mathrm{FeO}$ in Hole 396B basalts. Constant $\mathrm{Mg}^{\prime}$ reference lines and average glass compositions from sub-units $\mathrm{A}_{1}, \mathrm{~A}_{2}$, and $\mathrm{A}_{3}$ and Unit $\mathrm{C}$ are also included. Olivine control lines on this diagram are sub-horizontal with slightly negative slopes. Because large changes in $\mathrm{MgO}$ content occur in response to small amounts of olivine fractionation, distinguishing variations in $\mathrm{Mg}^{\prime}$ due to magmatic and alteration processes is difficult, particularly since alteration indexes do not always agree with the intensity of chemical change. However, the evidence that alteration among the aphyric lavas has produced much of the $\mathrm{MgO}$ variation is strong due to the relative paucity of phenocrysts. Magnesium loss has also occurred in the phyric rocks as fresh, relatively evolved samples from Unit B have $\mathrm{Mg}^{\prime}$ values around 0.63 to 0.64 and there are strongly altered samples with $\mathrm{Mg}^{\prime} \approx 0.5$.

\section{CHEMICAL STRATIGRAPHY AND PETROGRAPHY}

\section{Unit A}

The uppermost 88 meters of basaltic bedrock in Hole 396B are comprised of very sparsely phyric basalt (hereafter referred to as aphyric basalt for convenience). Three chemically defined sub-units are recognized $\left(A_{1}, A_{2}\right.$, and $\left.A_{3}\right)$. This breakdown is consistent for major, minor, and trace element analyses of bulk rocks as well as microprobe analysis of residual glasses in pillow rinds. As discussed previously, the major element chemistry of these rocks has been disturbed to varying degrees by alteration caused by basalt-seawater interaction. However, relatively non-labile, magmaphile elements such as $\mathrm{Ti}, \mathrm{Zr}, \mathrm{Y}$, and $\mathrm{REE}$ are excellent indicators for the purpose of defining individual units (Table 3 ). The stratigraphic sub-units of A, recognized here on the basis of bulk and glass chemistry, are the same as originally defined on the basis of shipboard analyses and generally are supported by inferred lithologic breaks and magnetic reversal stratigraphy.

The stratigraphic-chemical progression, upsection from $A_{3}$ to $A_{1}$, is from more evolved at the base $\left(A_{3}\right)$ to a more "primitive" composition $\left(\mathrm{A}_{1}\right)$ at the top, with $\mathrm{A}_{2}$ being intermediate in stratigraphic position and chemistry. Within 


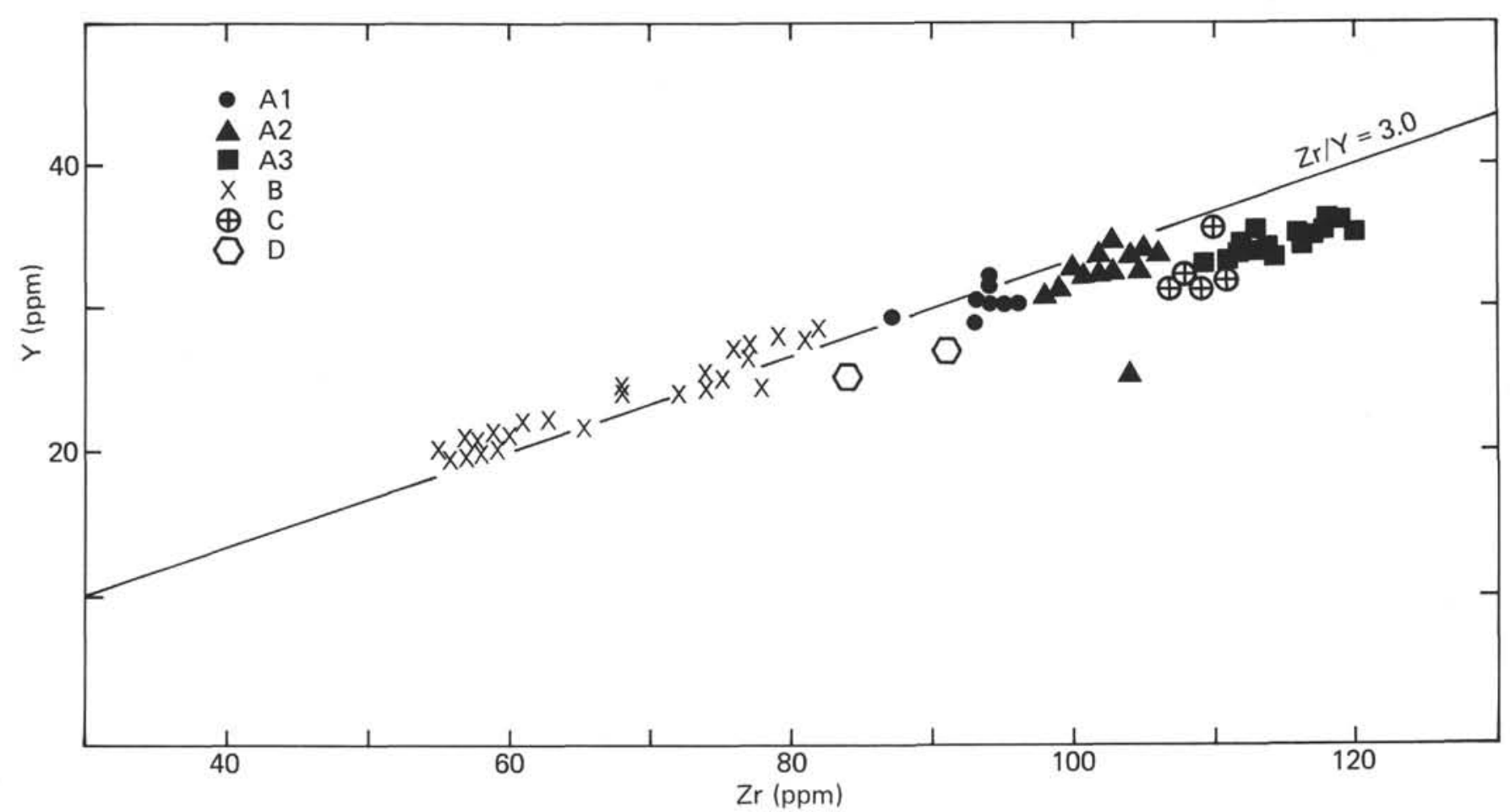

Figure 4. Zirconium-yttrum relationships in basalts of Hole 396B. The reference here is for a constant ratio of 3.0.

this progression $\left(\mathrm{A}_{3} \rightarrow \mathrm{A}_{2} \rightarrow \mathrm{A}_{1}\right), \mathrm{TiO}_{2}, \mathrm{Na}_{2} \mathrm{O}, \mathrm{FeO}$, and total REE all decrease whereas $\mathrm{CaO}, \mathrm{MgO}$, and $\mathrm{Mg} /(\mathrm{Mg}+\mathrm{Fe})$ increase. A second subdivision between $\mathrm{A}_{1}$ and $A_{2}$ on the one hand, and $A_{3}$ on the other, is recognized on the basis of $\mathrm{CaO} / \mathrm{Al}_{2} \mathrm{O}_{3}$ in glassy pillow rinds. Both $\mathrm{A}_{1}$ and $\mathrm{A}_{2}$ have $\mathrm{CaO} / \mathrm{Al}_{2} \mathrm{O}_{3}$ of 0.77 to 0.76 , in contrast with $\mathrm{A}_{3}$ which has $\mathrm{CaO} / \mathrm{Al}_{2} \mathrm{O}_{3}=0.74$. Basalts in Sub-unit $\mathrm{A}_{3}$ have lower $\mathrm{CaO}$ than those in $\mathrm{A}_{1}$ and $\mathrm{A}_{2}$ but their $\mathrm{Al}_{2} \mathrm{O}_{3}$ contents are intermediate. In addition, the stable magnetic inclinations of $\mathrm{A}_{1}$ and $\mathrm{A}_{2}$ are approximately $+15^{\circ}$, as opposed to the $-70^{\circ}$ measured for $\mathrm{A}_{3}$.

Textures of the rapidly cooled parts of $\mathrm{A}_{1}, \mathrm{~A}_{2}$, and $\mathrm{A}_{3}$ are similar in that all three contain microphenocrysts of olivine and plagioclase on eruption. Pyroxene occurs only as a groundmass phase; chromian spinel is absent. The major textural-mineralogical difference among the units is that $\mathrm{A}_{1}$ and $A_{2}$ are composed entirely of pillow basalt and $A_{3}$ is a 10-meter cooling unit with a pillowed carapace and a massive interior that is substantially more coarse grained than the pillow interiors in $A_{1}$ and $A_{2}$. Kirkpatrick (this volume) describes the textural progression from glassy pillow margin to intersertal pillow interior. However, it can be noted here that the combination of plagioclase spherulites grading into more slowly cooled intersertal basalt is characteristic of multiply saturated ocean floor tholeiites. Chemically similar basalts in Holes 395 and 395A that have olivine alone on the liquidus are characterized by substantially different textures (Dungan et al., 1978).

All three sub-units also contain sparse anhedral grains of plagioclase and olivine that are distinctly larger than the microphenocrysts. In contrast to skeletal and euhedral morphologies of the microphenocrysts, these grains are generally rounded and embayed suggesting resorption and disequilibrium. As these grains have compositions different from those of the microphenocrysts as well, they are inferred to have crystallized from more primitive melts or in some cases more evolved melts than those in which they are now found.

Sample $396-14-6,94-100 \mathrm{~cm}$ is the stratigraphically highest basalt analyzed by us from Hole 396 . It is representative of a small amount of aphyric basalt mixed with foraminiferal ooze at the basement-sediment interface. Its major element composition resembles that of $\mathrm{A}_{2}$ (Hole 396B) and may be equivalent. However, the magnetic inclination of this sample is $-20^{\circ}$ which does not agree with that of any of the sub-units of A (Hole 396B). These magnetic data, the small thickness of aphyric basalt, and the high degree of alteration of this sample suggest that this is a loose block derived from adjacent flows of A.

\section{Unit B}

Underlying Unit $\mathrm{A}$ is a section of abundantly phyric pillow lavas $(61 \mathrm{~m})$. These are distinct, both chemically and petrographically, from the aphyric Unit A. Plagioclase and olivine phenocrysts are abundant (10 to $20 \%$ ) and, in contrast to A, minor chromian spinel is present. Spinel crystals are found as inclusions in both plagioclase and olivine and as microphenocrysts in the groundmass where they typically have a vermiform habit. Clinopyroxene appears only as a groundmass phase. Although Unit B is a coherent stratigraphic and magnetic unit, it encompasses substantial chemical variability. On the basis of chemical analyses onboard ship and in this study, a chemical break in Unit B is recognized in Core 20 between Sections 2 and 3, the lower unit being the more primitive. This difference is also present in the residual, glassy selvages above and 
below the break defined by the bulk analyses. However, there are also gradational chemical changes throughout Unit $\mathrm{B}$ in the same sense as those which define the discontinuous change in Core 20. This is in contrast to the Sub-units of A which exhibit very slight internal variation.

The absolute chemical changes from the base to the top of the unit are as follows: (1) $\mathrm{Na}_{2} \mathrm{O}, \mathrm{FeO}$, and $\mathrm{TiO}_{2}$ increase more or less regularly except for minor perturbations and the aforementioned discontinuity; (2) $\mathrm{MgO}$ and $\mathrm{Mg} /(\mathrm{Mg}+\mathrm{Fe})$ decrease; (3) $\mathrm{CaO}$ and $\mathrm{Al}_{2} \mathrm{O}_{3}$ are variable from sample to sample, but do not define clearcut trends from base to top (however, $\mathrm{CaO}$ and $\mathrm{Al}_{2} \mathrm{O}_{3}$ correlate, suggesting that irregular plagioclase distribution is the cause for the lack of systematic variation); and (4) $\mathrm{SiO}_{2}, \mathrm{~K}_{2} \mathrm{O}$, and $\mathrm{P}_{2} \mathrm{O}_{5}$ tend to increase upward with substantial scatter that may be related in part to degree of alteration and/or analytical precision.

The sense of the chemical trends in Unit B, with respect to stratigraphic position, are the reverse of these among the sub-units of A, i.e., the more evolved compositions occur at the base rather than at the top. These generalizations have been made on the basis of only relatively fresh samples because many of the Unit B phyric rocks have undergone chemical alterations similar to these recognized in Unit A. Analyses labeled "glass" in Table 1 are glassy pillow rinds containing crystals in equivalent amounts to the pillow interiors and are not glass analyses as such. These are the least altered samples available, and considerable reliance has been placed on these for both classification and petrologic purposes.

The vast majority of the basalts in Hole 396 are phyric pillow lavas essentially equivalent in petrography, mineral chemistry, and chemical composition to the upper part of Unit B (Hole 396B). However, the magnetic inclinations of the phyric basalts in Core 14 through the upper portion of Core 22 (Hole 396 ) are $\approx+40^{\circ}$, as opposed to the remainder of Hole 396 and the entire thickness of Unit B (Hole 396B) which is $-10^{\circ}$. Thus, the lower portion of Hole 396 probably correlates directly with Unit B (Hole 396B); Unit $\mathrm{A}$ is all but absent and there is an additional phyric unit at the top of Hole 396 that is lacking in Hole 396B.

\section{Unit C}

The lowermost 90 meters of drilling in Hole 396B penetrated sparsely phyric basalt that occurs as fragments in what appears to be a clastic unit rather than a coherent sequence of pillows. This inference is based on the low recovery achieved in this depth interval, extensive caving of the walls and bedded hyaloclastites (Site Report, this volume) that are present in the recovered material. With the exception of some fragments of phyric basalt in Core 32 ("Unit D') that are petrographically and chemically distinct, the sparsely phyric basalts within this clastic interval (Cores 23 to 32 ) are chemically coherent. They generally resemble the $A_{2}$ and $A_{1}$ basalts in composition, but there are small, systematic differences in both major and trace elements. Although $\mathrm{C}$ and $\mathrm{A}_{2}$ have essentially identical $\mathrm{TiO}_{2}$ and $\mathrm{CaO}$ contents, the Unit $\mathrm{C}$ basalts are characterized by higher $\mathrm{Al}_{2} \mathrm{O}_{3}, \mathrm{FeO}, \mathrm{Na}_{2} \mathrm{O}, \mathrm{K}_{2} \mathrm{O}, \mathrm{Sr}$, $\mathrm{La} / \mathrm{Sm}, \mathrm{Zr}$, substantially higher $\mathrm{Nb}$, and slightly lower $\mathrm{SiO}_{2}$. Again, detailed comparisons must be made on the relatively few unaltered samples that have not undergone major element redistribution due to interaction with seawater.

\section{MINERAL CHEMISTRY}

\section{Olivine}

Unit A contains two petrographic types of olivine: (1) abundant microphenocrysts and (2) sparse, larger anhedral grains. The microphenocrysts in Unit A are progressively more magnesian from $A_{1}$ to $A_{2}$ to $A_{3}$ in accordance with the change in bulk chemistry. Microphenocryst compositions in the three sub-units are $A_{3}=F 084.3 \pm 0.3, A_{2}=$ Fo85.0 0.3 , and $A_{1}=$ Fo86.1 \pm 0.3 . The larger, anhedral crystals do not exhibit this compositional correlation; some of these large grains are more magnesian than the associated microphenocrysts, whereas others are more iron rich. The latter are conspicuously evidenced by reverse zoning accompanied by irregular rim overgrowths on the anhedral cores. Olivine microphenocrysts in Unit $\mathrm{C}$ are similar in composition to those in $\mathrm{A}_{1}$ (Fo86.2 \pm 0.3 ) in accordance with the very similar bulk compositions of the two units. One of the two samples of $\mathrm{C}$ from which we have analyzed olivine contains a glomerophyric aggregate of plagioclase plus olivine of the composition Fo88-87.

The compositional variation of olivine in the phyric Unit $\mathrm{B}$ is much larger and more complex. Figure 5 illustrates the relationships between olivine composition and coexisting melt composition. Roeder and Emslie (1970) have shown that olivine composition is a function of the $\mathrm{Mg} /\left(\mathrm{Mg}+\mathrm{Fe}^{+2}\right)$ of the liquid from which it crystallizes. Reference curves for $\mathrm{Fe}-\mathrm{Mg}$ partitioning between olivine and liquid are also plotted on Figure 5. Three olivine-liquid relationships are shown by different symbols. In the first, microphenocrysts in aphyric and porphyritic basalts are plotted against the coexisting residual glass composition (both mineral and glass data are microprobe analyses). These data define a KD of 0.27 for the range of $\mathrm{Mg} /\left(\mathrm{Mg}+\mathrm{Fe}^{+2}\right)$ from 0.58 and 0.64 . This value is presumed to be the equilibrium $\mathrm{Fe}-\mathrm{Mg}$ partition coefficient for olivine and liquid in these rocks. The second set of data is comprised of a total of 70 olivine analyses from four porphyritic basalts from Unit B plotted against the bulk rock composition (Table 1). Most of the analyses represent core compositions of phenocrysts although rims also were analyzed. With the exception of rare, reversely zoned olivine crystals (triangles), the most iron-rich compositions in each rock are those microphenocrysts coexisting with residual glass whose compositions yield a $K_{D}$ of 0.27 . Under equilibrium conditions, the most magnesium olivine predicted for those rocks also should give a KD of 0.27 . On the contrary, many of the phenocrysts are more forsteritic than predicted by this method. As these more magnesian crystals must have had a marked effect in changing the $\mathrm{Mg} /\left(\mathrm{Mg}+\mathrm{Fe}^{+2}\right)$ toward higher values, the present bulk rock compositions must be a combination of a liquid ( \pm plagioclase crystals) that have gained some magnesian olivine phenocrysts, making minimal the discrepancy between predicted and observed olivine compositions. A similar relationship was recognized in porphyritic basalts of Holes 395 and 395A (Dungan et al., 1978). In addition to the disequilibrium indicated by these forsteritic phenocrysts, there are also phenocrysts that 


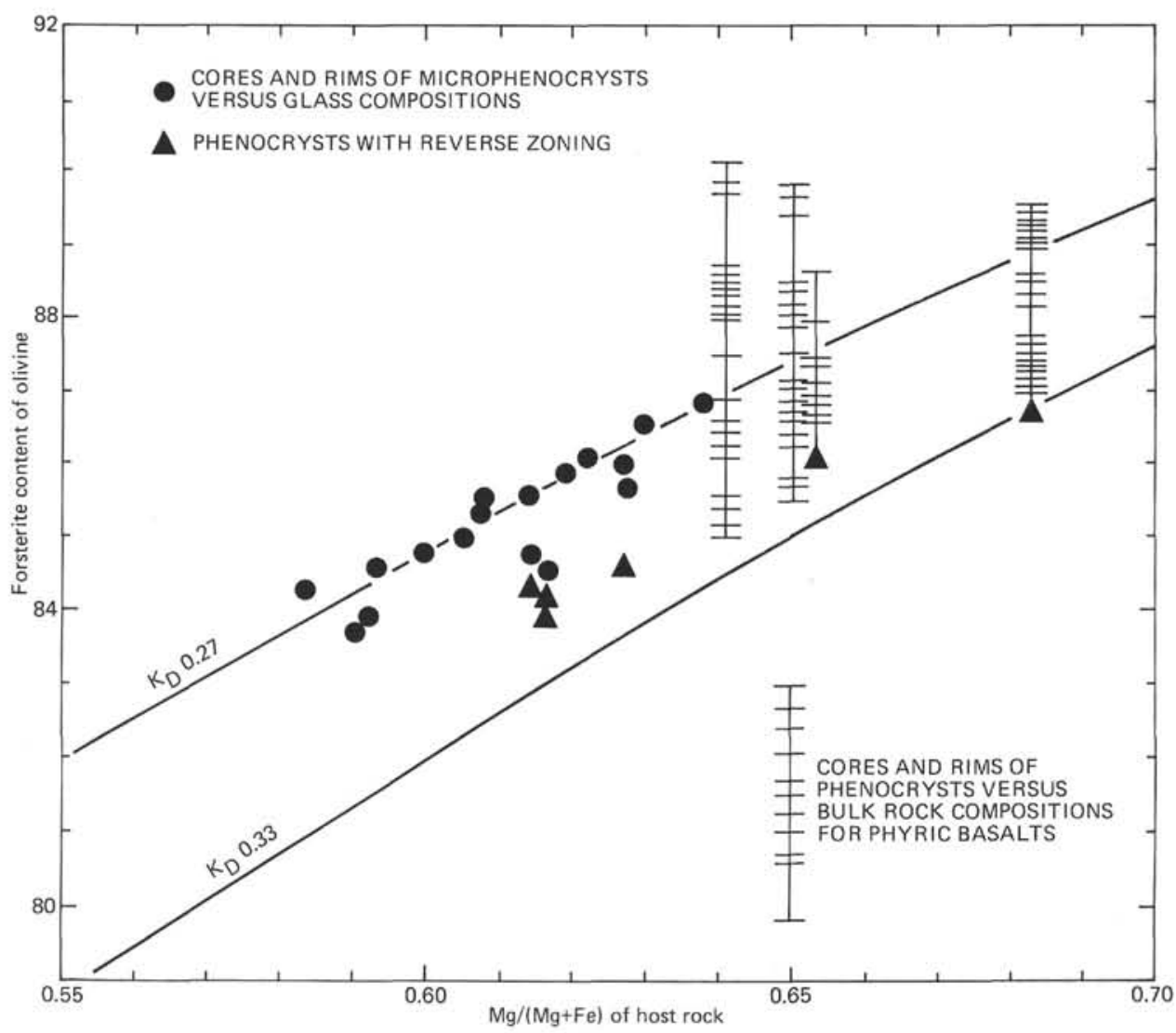

Figure 5. Plot of olivine and "coexisting liquid" for microphenocrysts in glass and phenocrysts and their bulk rock composition. Reference lines for Fe-Mg partitioning values $\left(K_{D}\right)$ of 0.27 and 0.33 are calculated after Roeder and Emslie (1970).

show reverse zoning from cores more iron rich than the microphenocrysts in both aphyric and phyric lavas.

\section{Plagioclase}

One-atmosphere melting experiments on three natural rock powders representative of the three chemical units of Hole 396B indicate that plagioclase is the liquidus phase in all three rock types. The aphyric rocks from $\mathrm{A}$ and $\mathrm{C}$ are shown to be essentially cotectic, with olivine and plagioclase appearing within 5 to $10^{\circ} \mathrm{C}$ of each other. In the porphyritic sample (Unit B), spinel and then olivine follow plagioclase in the crystallization sequence over a temperature interval of about $35^{\circ} \mathrm{C}$. Compositions of the phases occurring in the experimental runs are shown in Figure 6 and Table 6.

Microprobe analyses of natural plagioclases in a few of the Leg 46 basalts and in the experimentally produced charges are three element analyses (K-Ca-Na) that have been converted directly to molecular end-member components without matrix corrections. The use of natural standards with compositions near the unknowns results in close approximations to the actual values. Sub-units $A_{1}$ and $\mathrm{A}_{2}$ contain microphenocrysts of $\mathrm{An}_{65-68}$, and $\mathrm{A}_{3}$ has more sodic plagioclases of An61-65. However, the larger anhedral grains which typically exhibit textural evidence of resorption are more calcic (An74-86) than the microphenocrysts. A similar situation obtains in Unit C which has microphenocrysts of An71-74 and larger euhedral and anhedral grains that are as calcic as An84. Only one porphyritic basalt (Sample 396B-22-3, 28-38 cm) has been analyzed in reconnaissance for plagioclase compositions. A pattern very much like the porphyritic Leg 45 basalts (Dungan et al., 1978) is present. Rim compositions in glass are An69-72 and core compositions of phenocrysts range from $\mathrm{An}_{75}$ to An85. Many additional analyses are needed to fully document the range of compositions in the phyric rocks, particularly since there is a diversity of zoning patterns present. Additional studies currently are being conducted.

\section{Spinel}

Chromian spinel has been recognized as a microphenocryst phase only in phyric basalts in Holes 396 and 396B. It occurs throughout Unit B and in both clasts of the phyric basalt in Core 32 ("Unit D"). The single occurrence of spinel in either aphyric unit is an included grain in a glomerophyric aggregate of calcic plagioclase (An85-83) in Sample 396B-14-1, 21-25 cm (Sub-unit A $\mathrm{A}_{3}$; JSC No. 209). Table 4 contains some representative microprobe analyses of spinels in these rocks and Figure 7 is a $\mathrm{Cr} /(\mathrm{Cr}+\mathrm{Al})$ versus $\mathrm{Mg} /(\mathrm{Mg}+\mathrm{Fe})$ plot of 70 analyses from basalts in Unit B (Hole 396B). An additional 20 analyses from phyric basalts in Hole 396 span the same range of compositions as those from 396B, as do those from phyric basalts in Hole 395. The one-atmosphere phase relations of 
$3968-7 \cdot 1,42-46 \mathrm{~cm}$

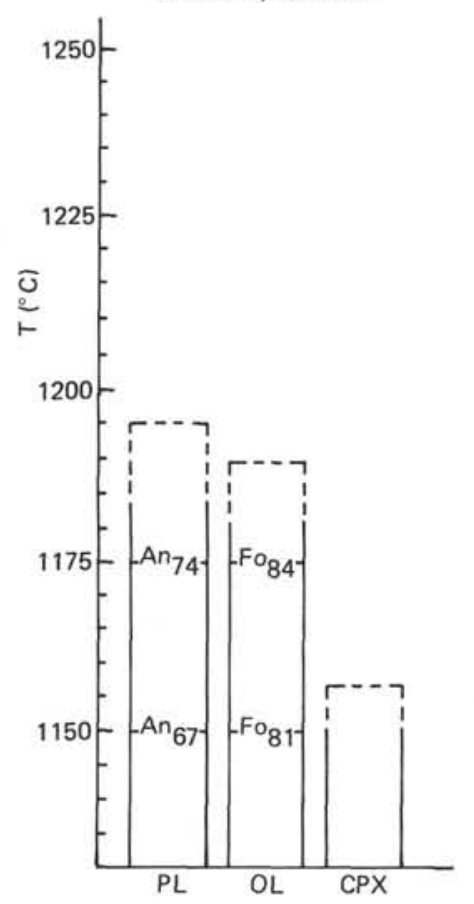

$396 \mathrm{~B}-30-1,77-80 \mathrm{~cm}$

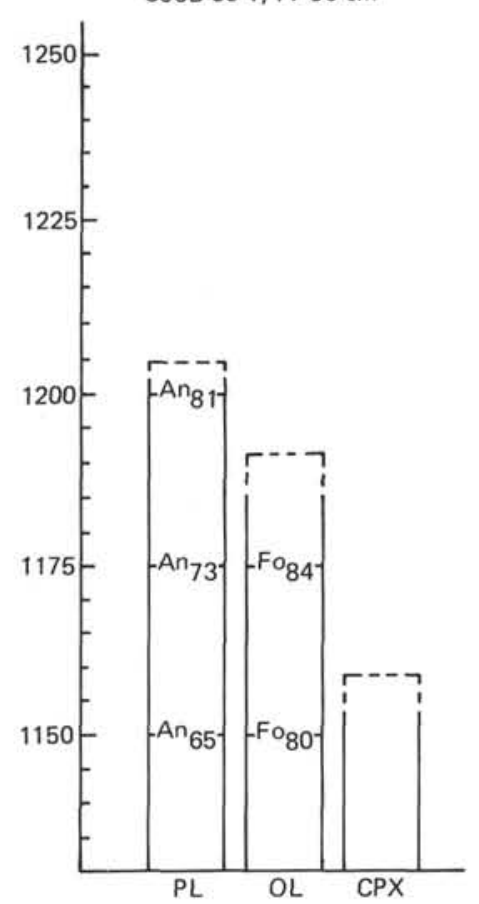

$3968-22-3,28-38 \mathrm{~cm}$

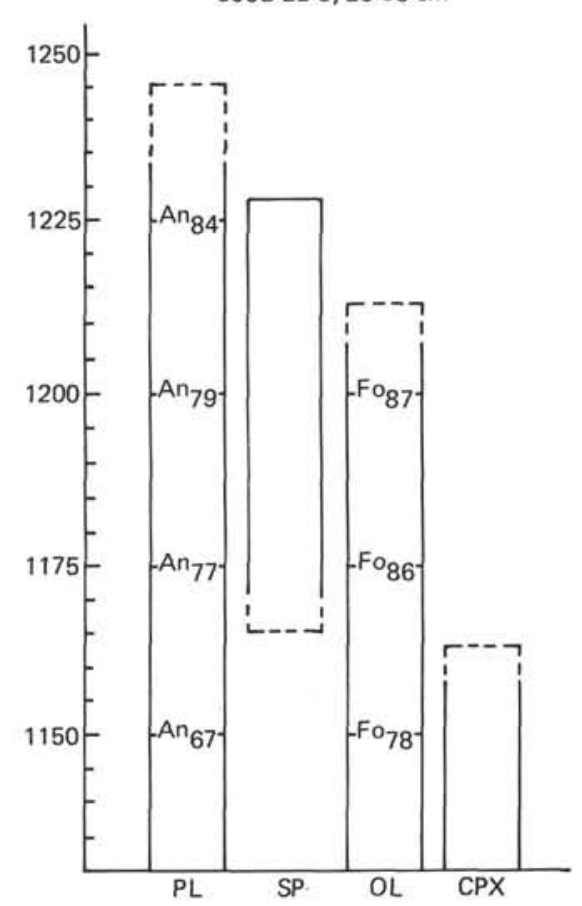

Figure 6. Mineralogy as function of temperature produced in one-atmosphere melting experiments on three select basalts from Hole 396B. These three are representative of the three major basalt units.

Sample 396B-22-3, 28-38 cm indicate that spinel is a near liquidus phase and is stable over at least $50^{\circ} \mathrm{C}\left(1225^{\circ}\right.$ to $\left.1175^{\circ} \mathrm{C}\right)$. This is a somewhat larger temperature interval than was found in melting experiments with Sample $395 \mathrm{~A}-27-2,111-116 \mathrm{~cm}$, a phyric basalt that is similar in composition.

In accordance with its larger stability field in the Hole 396 B composition, spinel is more abundant in the phyric basalts of Unit B (396B) than in any of the phyric units at Site 395. The spinels at these two sites are similar to those analyzed in other moderately evolved ocean floor tholeiites $\left(\mathrm{TiO}_{2}<1.5 \%, \mathrm{Cr} /(\mathrm{Cr}+\mathrm{Al})=0.3\right.$ to 0.6 , and $\mathrm{Mg} /(\mathrm{Mg}+\mathrm{Fe})$ $=0.7$ to 0.45 ). Highly aluminous, chrome-poor spinels, such as those which occur in rare, magnesian, primitive tholeiites that have been recovered from some localities of the Atlantic sea floor (Frey et al., 1974; Sigurdsson and Schilling, 1976) are not present.

As noted on Figure 5, the spinel crystals in the Hole 396B basalts occur as inclusions within olivine and plagioclase phenocrysts and as isolated grains within the groundmass. Spinel crystals that are attached to the outer surfaces of grains in glomerophyric aggregates are classed as groundmass grains. The included grains are euhedral or subhedral and typically contain small $(<10 \mu \mathrm{m})$ melt inclusions indicating that they are magmatic crystals. The groundmass grains exhibit two distinct morphologies. Many are equant and euhedral to subhedral, whereas others have a vermiform habit. The latter texture is somewhat enigmatic in that it may signify resorption or it may form as a function of rapid growth, i.e., analogous to skeletal growth in silicates.
There is a correlation between petrography and chemical variations within the composite of spinel analyses from Unit B. The groundmass spinels have $\mathrm{Cr} /(\mathrm{Cr}+\mathrm{Al})$ ratios within the higher part of the range defined by those that occur as inclusions, particularly in plagioclase. Spinel crystals included in olivine phenocrysts and in the groundmass have lower $\mathrm{Mg} /(\mathrm{Mg}+\mathrm{Fe})$ compared to those within plagioclase. The generally higher $\mathrm{Cr} /(\mathrm{Cr}+\mathrm{Al})$ and lower $\mathrm{Mg} /(\mathrm{Mg}+\mathrm{Fe})$ of the groundmass spinels, as compared to the inclusions, mirrors the limited zoning found in individual grains (from core to rim). Similarly, analyses of spinels produced experimentally at $1225^{\circ}$ and $1200^{\circ} \mathrm{C}$ show the same trend. These are displaced to higher $\mathrm{Mg} /(\mathrm{Mg}+\mathrm{Fe})$ for a given $\mathrm{Cr} /(\mathrm{Cr}+\mathrm{Al})$ than the natural spinels. This is probably a result of the composite nature of the rocks in that they have gained olivine phenocrysts, thus producing a bulk liquid composition with an artificially high $\mathrm{Mg} /(\mathrm{Mg}+\mathrm{Fe})$.

\section{Microprobe Analyses of Residual Glasses and Melt Inclusions}

An extensive survey of natural glass compositions in aphyric and porphyritic basalts from Sites 395 and 396 (Legs 45 and 46) has been undertaken. Eleven element microprobe analyses, corrected for matrix effects by the Bence-Albee method, are presented in Table 4. These include glassy selvages from quenched pillow rinds (residual glasses) and glass trapped in plagioclase and olivine phenocrysts (melt inclusions). Four three-element passes were made on each residual glass selvage or melt inclusion. For the selvages and larger melt inclusions $(>25$ $\mu \mathrm{m}), 10$ to 12 points were counted for 20 to 30 seconds and 


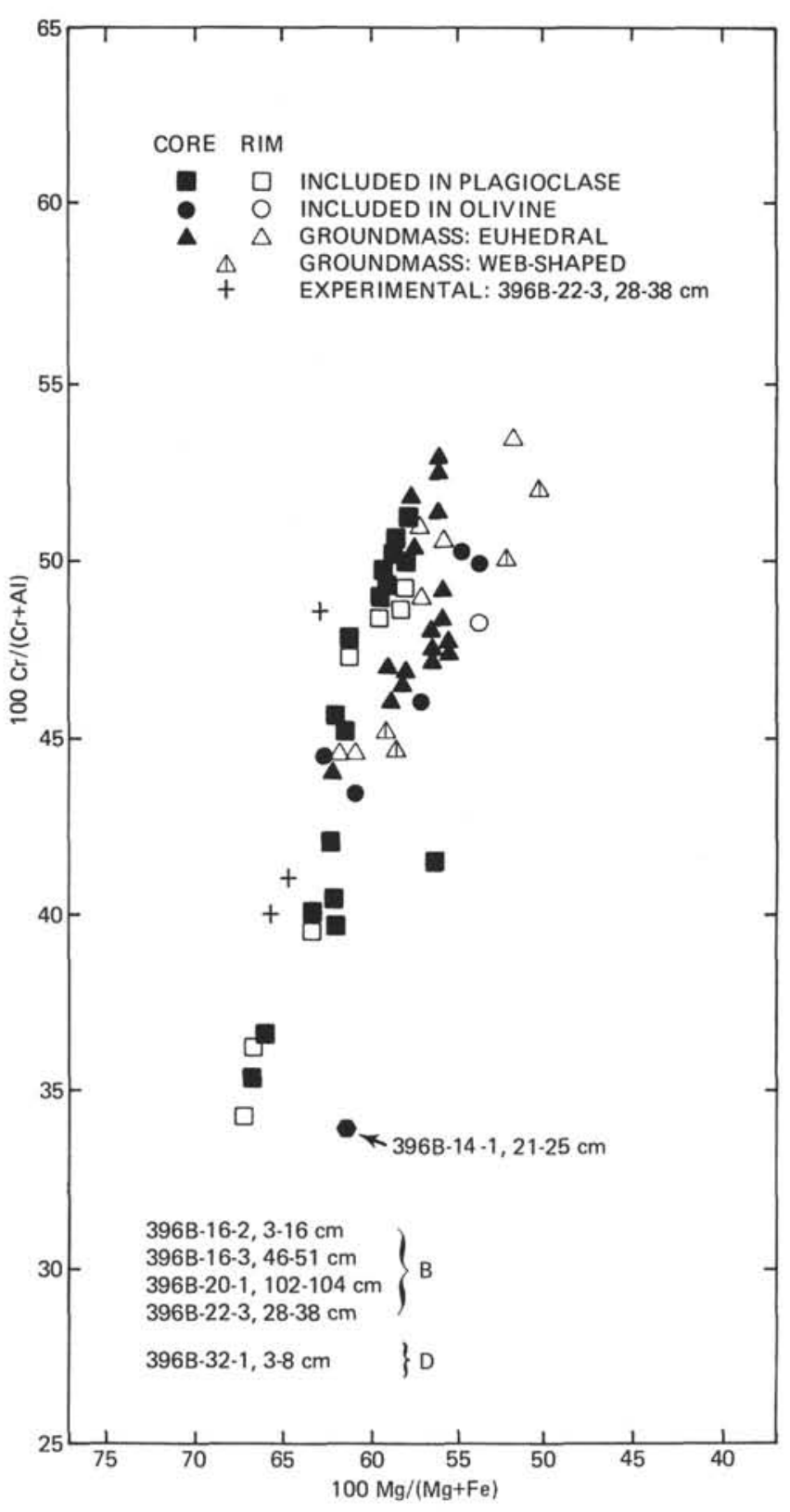

Figure 7. $\mathrm{Cr} /(\mathrm{Cr}+\mathrm{Al})$ versus $\mathrm{Mg} /(\mathrm{Mg}+\mathrm{Fe})$ in chromian spinels of Site 396 phyric basalts.

then averaged. The residual glasses and melt inclusions are discussed separately below.

\section{Residual Glasses}

We have found evidence at both Site 395 (Dungan et al., 1978) and Site 396 suggesting the phyric basalts are comprised of mixtures of at least two liquids and their crystals. The critical part of this evidence is the presence of phenocrysts that are not in equilibrium with the liquids in which they occur or, more importantly, with liquids corresponding to the bulk rock compositions. Consequently, bulk rock analyses of the phyric basalts cannot be treated in petrologic calculations as liquids. The residual glasses are then the only available material that represent relevant liquid compositions. Microprobe analyses of natural glass circumvents another problem that hampers interpretation of bulk rock chemical data in that they have not been modified by interaction with seawater as have most of the rocks.

One of the most striking compositional characteristics of the residual glasses at Sites 395 and 396 is the similarity of the glasses associated with aphyric basalts (which approximate bulk rock compositions) and the residual glasses in the phyric rocks. This similarity is evidenced by the elemental ratio $\mathrm{Mg} /\left(\mathrm{Mg}+\mathrm{Fe}^{+2}\right)$ which is a sensitive indicator of olivine fractionation. At both sites, the residual glasses associated with aphyric and phyric basalts have essentially the same $\mathrm{Mg} /\left(\mathrm{Mg}+\mathrm{Fe}^{+2}\right)$. This is illustrated by Figures 7 and 8 in which $\mathrm{TiO}_{2}, \mathrm{Al}_{2} \mathrm{O}_{3}, \mathrm{CaO}, \mathrm{CaO} / \mathrm{Al}_{2} \mathrm{O}_{3}$, and $\mathrm{Na}_{2} \mathrm{O}$ are plotted against $\mathrm{Mg} /\left(\mathrm{Mg}+\mathrm{Fe}^{+2}\right)$. The glasses at Site 395 are more evolved, i.e., lower $\mathrm{Mg} /\left(\mathrm{Mg}+\mathrm{Fe}^{+2}\right)$ and higher $\mathrm{TiO}_{2}$, than those at Site 396; the differences between phyric and aphyric are larger for a given $\mathrm{Mg} /\left(\mathrm{Mg}+\mathrm{Fe}^{+2}\right)$ than at Site 396. However, in terms of elemental trends within related groups of rocks and the sense of the compositional differences between the phyric and aphyric glasses, the same relationships hold at both sites.

The most primitive glasses are those from the lower part of Unit B (Section 396B-20-3), but those from the upper part of this unit have $\mathrm{Mg} /\left(\mathrm{Mg}+\mathrm{Fe}^{+2}\right)$ values that overlap with $\mathrm{A}_{1}$ and $\mathrm{A}_{2}$. Both groups have the same $\mathrm{K}_{2} \mathrm{O}, \mathrm{Al}_{2} \mathrm{O}_{3}$, and $\mathrm{SiO}_{2}$. However, the glasses in aphyric rocks have higher $\mathrm{TiO}_{2}$ and $\mathrm{Na}_{2} \mathrm{O}$, and lower $\mathrm{CaO}$. The four residual glasses from Site 395 exhibit the same compositional pattern with respect to the single analysis of glass from the evolved aphyric Sub-unit $\mathrm{A}_{3}$ (Hole 395A).

Unit C (Hole 396B), which is comprised of sparsely phyric basalts, has trace element characteristics distinct from Units A and B. Its residual glass chemistry is very similar in general character to the upper aphyric unit (particularly Sub-units $\mathrm{A}_{1}$ and $\mathrm{A}_{2}$ ) but is distinct in detail. The Unit $\mathrm{C}$ glasses have the same $\mathrm{Mg} /\left(\mathrm{Mg}+\mathrm{Fe}^{+2}\right)$, $\mathrm{TiO}_{2}$, $\mathrm{CaO}$, and $\mathrm{FeO}$ as $\mathrm{A}_{2}$; but are higher in $\mathrm{Al}_{2} \mathrm{O}_{3}, \mathrm{Na}_{2} \mathrm{O}$, and $\mathrm{K}_{2} \mathrm{O}$, and lower in $\mathrm{CaO} / \mathrm{Al}_{2} \mathrm{O}_{3}$ and $\mathrm{SiO}_{2}$.

\section{Discussion of Residual Glass Data}

Fractional crystallization of the observed phenocryst phases (olivine and plagioclase) is not sufficient to relate residual glasses of the three chemical units of Hole 396B. A relationship between Unit $\mathrm{C}$ on the one hand and either $\mathrm{B}$ or A on the other has been ruled out on the basis of its contrasting trace element systematics. However, the ratios of magmaphile elements in Units A and B are nearly the same. These ratios vary in a way compatible with small amounts of crystal fractionation of the observed phenocrysts. That Units B and A are not related by fractional crystallization of olivine and plagioclase is clearly demonstrated by the glass compositions. All the glasses contain microphenocrysts of olivine. As the ratio $\mathrm{Mg} /\left(\mathrm{Mg}+\mathrm{Fe}^{+2}\right)$ is very sensitive to olivine fractionation, removal of olivine is precluded by the similar $\mathrm{Mg} /\left(\mathrm{Mg}+\mathrm{Fe}^{+2}\right)$ of the glasses. Plagioclase fractionation from the Unit B glasses would explain the higher $\mathrm{TiO}_{2}$ and lower $\mathrm{CaO}$ in the aphyric glasses (at a fixed 


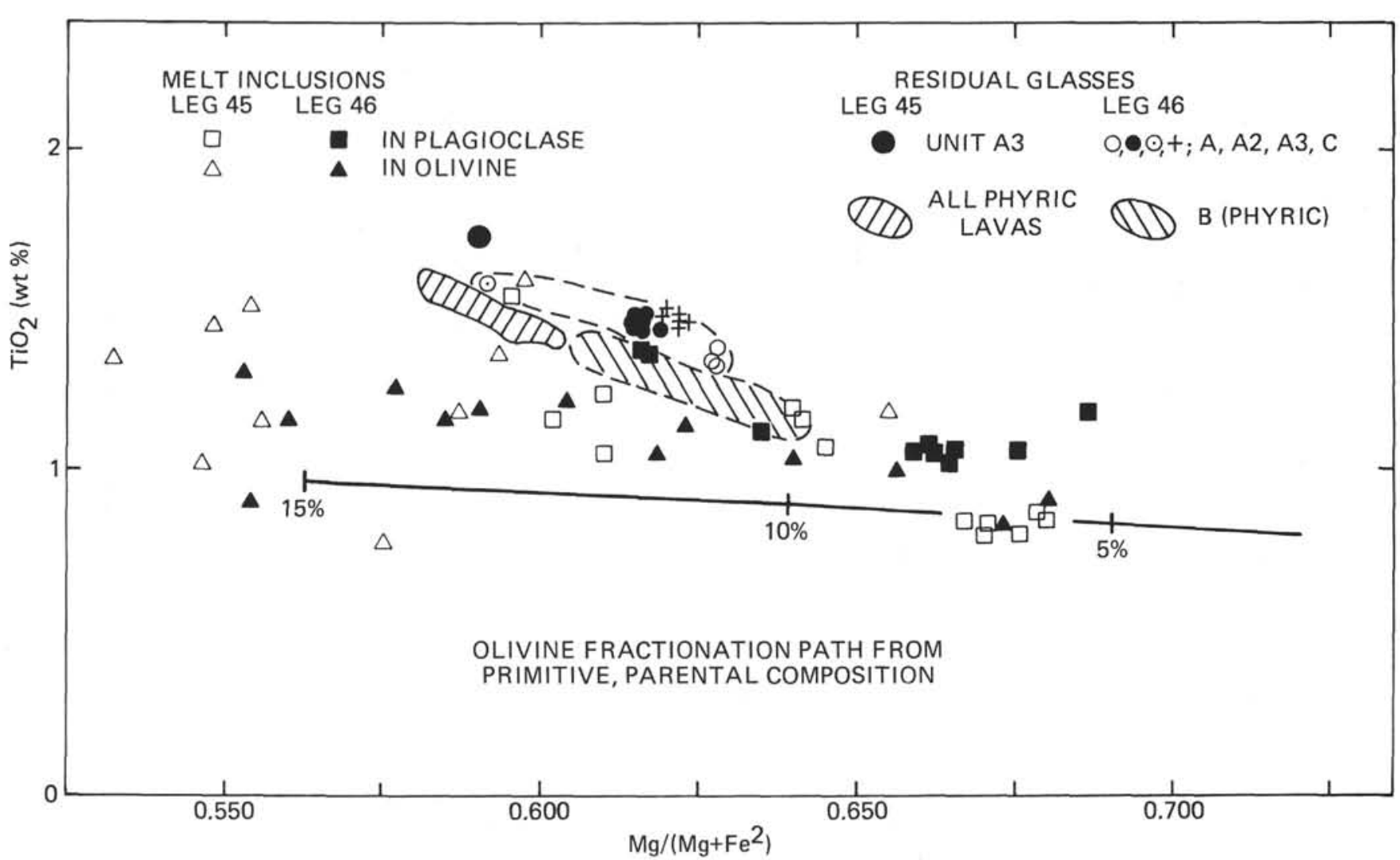

Figure 8. $\mathrm{TiO}_{2}$ versus $\mathrm{Mg}^{\prime}$ relationships in natural residual glasses and glass melt inclusions from basalts of Sites 395 and 396.

$\mathrm{Mg} /\left(\mathrm{Mg}+\mathrm{Fe}^{+2}\right)$, but this cannot be accomplished at a constant $\mathrm{Al}_{2} \mathrm{O}_{3}$ as is required by the compositional data. Clinopyroxene is an additional phenocryst phase in the Site 395 phyric basalts, and here again the oxide variations at a constant $\mathrm{Mg} /\left(\mathrm{Mg}+\mathrm{Fe}^{+2}\right)$ cannot be produced by fractional crystallization alone, although the trace element systematics again are compatible with a cogenetic relationship.

As shown on Figures 7 and 8 , there are systematic chemical trends within the two phyric groups and the sub-units of $\mathrm{A}$. In all cases, as $\mathrm{Mg} /\left(\mathrm{Mg}+\mathrm{Fe}^{+2}\right)$ decreases, the levels of $\mathrm{Na}_{2} \mathrm{O}, \mathrm{TiO}_{2}, \mathrm{FeO}$, and $\mathrm{SiO}_{2}$ increase and the $\mathrm{CaO} / \mathrm{Al}_{2} \mathrm{O}_{3}$ decreases. The increases in $\mathrm{TiO}_{2}$, etc. are to be expected from fractional crystallization of olivine and plagioclase, but the decrease in $\mathrm{CaO} / \mathrm{Al}_{2} \mathrm{O}_{3}$ is in the reverse sense to that caused by the subtraction of plagioclase. Clinopyroxene has a very high $\mathrm{CaO} / \mathrm{Al}_{2} \mathrm{O}_{3}$ ratio $(\approx 5)$ and the removal of small amounts of this phase could produce a decrease in this parameter. In the Site 395 phyric basalts, which do contain clinopyroxene phenocrysts, the decreasing $\mathrm{CaO} / \mathrm{Al}_{2} \mathrm{O}_{3}$ trend with decreasing $\mathrm{Mg} /\left(\mathrm{Mg}+\mathrm{Fe}^{+2}\right)$ is the most marked of any group. Where clinopyroxene is lacking as a phenocryst in Hole $396 \mathrm{~B}$, the role of this phase in a simple fractionation model is highly problematic.

\section{Melt Inclusions}

Relative to the residual glasses, the melt inclusions trapped within olivine and plagioclase phenocrysts exhibit substantial compositional variation. Part of the diversity and deviation from residual glass compositions is due to crystallization of the host phase from the trapped melt. This effect is more pronounced in those included in olivine than for melts within plagioclase. A second reason for the wide range of compositions is that the melt inclusions represent trapped liquids of varying parental composition. This is illustrated in Figure 7 by the wide range of $\mathrm{TiO}_{2}$ contents present in melt inclusions in olivine phenocrysts from both sites. An example of the effect of olivine fractionation of a likely parental liquid shows that the total variation in $\mathrm{TiO}_{2}$ is several times greater than the amount that can be accounted for by olivine fractionation. Note that several of the melt inclusions (in both plagioclase and olivine) have $\mathrm{TiO}_{2}$ contents less than 1.0 weight per cent and that values around 0.8 weight per cent $\mathrm{TiO}_{2}$ are not uncommon. As the lowest $\mathrm{TiO}_{2}$ in a phyric basalt from Hole $396 \mathrm{~B}$ has $\mathrm{TiO}_{2}=0.93$, these low $\mathrm{TiO}_{2}$ melt inclusions must be trapped liquids of an original parent composition more primitive than the composition of the bulk rock in which they occur. Similarly, many of the melt inclusions have $\mathrm{CaO} / \mathrm{Al}_{2} \mathrm{O}_{3}$ ratios greater than 0.83 which is the maximum value for the residual glasses. This feature is illustrated in Figures 9 and 10. Note that there is a broad correlation between decreasing $\mathrm{CaO} / \mathrm{Al}_{2} \mathrm{O}_{3}$ and increasing $\mathrm{TiO}$ among the melt inclusions. Although this trend mimics the trends defined by the individual basalt units (e.g., A, B, or Site 395 phyric basalts), it cannot be produced by any combination of olivine and plagioclase fractionation unless plagioclase is resorbed back into the liquid rather than removed.

Using the available compositional data from the melt inclusions and the constraint that a primary mantle derived melt should have $\mathrm{Mg} /\left(\mathrm{Mg}+\mathrm{Fe}^{+2}\right)=0.72$, an estimate of the 


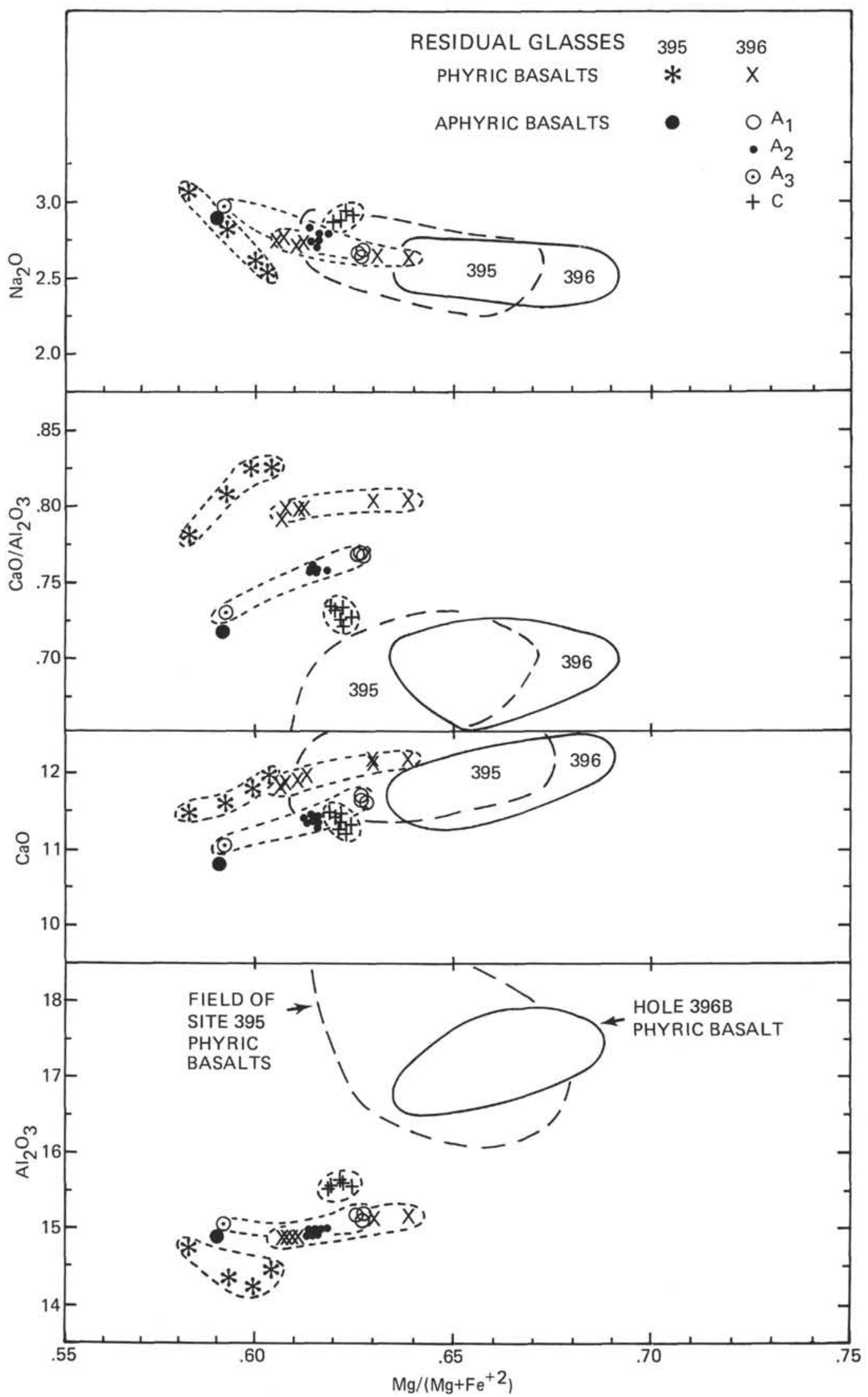

Figure 9. Compositional relationships among natural residual glasses and bulk rock at Sites 395 and 396. 


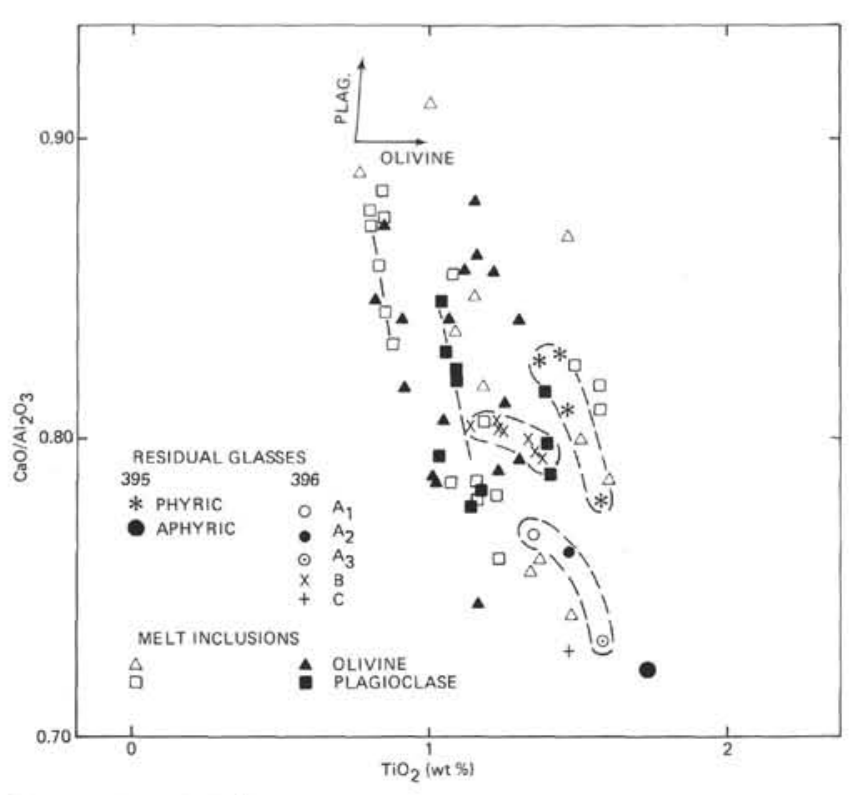

Figure 10. $\mathrm{CaO} / \mathrm{Al}_{2} \mathrm{O}_{3}$ versus $\mathrm{TiO}_{2}$ in natural residual glasses and bulk rock at Sites 395 and 396.

primitive composition has been graphically derived using oxide versus oxide and oxide versus oxide ratio plots. The following composition has been inferred on this basis: $\mathrm{SiO}_{2}$ $=49.0, \mathrm{TiO}_{2}=0.75, \mathrm{FeO}=8.3, \mathrm{MnO}=0.15, \mathrm{MgO}=$ $10.5, \mathrm{CaO}=13.5, \mathrm{Al}_{2} \mathrm{O}_{3}=15.0, \mathrm{Na}_{2} \mathrm{O}=1.9, \mathrm{~K}_{2} \mathrm{O}=$ 0.05 , and $\mathrm{P}_{2} \mathrm{O}_{5}=0.05$. This model magma remains to be tested by quantitative calculations involving fractionation of melt inclusions by crystallization of the host phase, but the inferred composition very closely resembles picritic ocean floor tholeiites from other localities (Frey et al., 1974; Blanchard et al., 1976; Bryan and Moore, 1976; Rhodes, unpublished data) that have been interpreted as primary mantle melts. A very significant geochemical characteristic of these primitive magmas is the high $\mathrm{CaO} / \mathrm{Al}_{2} \mathrm{O}_{3}$. The tendency for lower $\mathrm{CaO} / \mathrm{Al}_{2} \mathrm{O}_{3}$ with increasing fractionation and the apparent need for clinopyroxene fractionation is similarly an important feature of the residual glasses.

\section{EXPERIMENTAL DATA}

\section{Introduction}

Three representative samples of Leg 46 basalts have been selected for determination of their one-atmosphere phase relations and mineral compositions. The purpose of these experiments was to constrain the possible crystallization and fractionation histories of the basalts at the Leg 46 site and to determine if the phenocrysts in the natural rocks have compositions consistent with growth from their host. In addition, we have performed controlled cooling-rate experiments to establish a basis for the interpretation of textures in the natural basalts. The preliminary results of the experiments reported here allow us to draw several significant conclusions regarding the evolution and emplacement of basalts at Site 396.

\section{Experimental Techniques}

Both the melting and cooling rate experiments were performed using the technique described in detail by Donaldson et al. (1976) in which samples are suspended on a $\mathrm{Pt}$ wire loop in a vertical tube furnace. Oxygen fugacity in the furnace was controlled by mixing $\mathrm{H}$ and $\mathrm{CO}_{2}$ and was measured using a modified Sato cell calibrated against the $\mathrm{Ni}-\mathrm{NiO}$ buffer curve. In all the melting experiments, $\mathrm{f}_{\mathrm{O}_{2}}$ was maintained at $0.5( \pm 0.1) \log$ unit below the QFM buffer curve. The temperatures of the furnace are believed to be accurate within $3^{\circ} \mathrm{C}$. Approximately $100 \mathrm{mg}$ of finely ground rock powder was used for each run. For the melting runs, the sample was held at temperature for 24 to 72 hours and then quenched. The cooling-rate runs were held at $1250^{\circ} \mathrm{C}$ for 24 hours then cooled to $1000^{\circ} \mathrm{C}$ at rates of $2^{\circ}$, $10^{\circ}$, and $30^{\circ} \mathrm{C}$ per hour.

The experimental conditions described above do not duplicate conditions on the sea floor, at the time of eruption; e.g., there was roughly $0.5 \mathrm{~kb}$ of hydrostatic pressure on the rocks at that time. Thus, the conclusions we draw from these experiments are based on the assumptions that a small pressure difference and the presence of seawater in the natural environment would not significantly alter the phase relations, textures, or mineral chemistry relative to the natural system. We feel these assumptions are valid based on available high pressure data for basaltic systems (e.g., see Fujii et al., 1978) and the probable kinetic factors limiting the interaction of basalt and seawater. The $f_{0,}$ values used were based on a compilation of estimated $\mathrm{T}$ versus $\mathrm{f}_{0_{2}}$ data for deep-sea basalts.

\section{Experimental Results}

The results of the melting experiments are presented in Figure 6, and the compositions of plagioclase and olivine are tabulated as a function of temperature in Table 6. All three samples studied have plagioclase as the liquidus phase, but the sample from Unit B (396B-22-3, 28-38 cm) has a significantly higher liquidus than the two aphyric rocks. Plagioclase appears in Sample 396B-22-3, 28-38 cm at about $1245^{\circ} \mathrm{C}$. At $1225^{\circ} \mathrm{C}$, plagioclase (An84) coexists with chrome spinel; by $1200^{\circ} \mathrm{C}$ olivine (Fos7) joins the spinel and plagioclase (An79). This same mineral assemblage still coexists with liquid at $1175^{\circ} \mathrm{C}$ at which temperature the plagioclase composition is $\mathrm{An}_{77}$ and that of olivine is Fo86. At $1150^{\circ} \mathrm{C}$, spinel is no longer present; instead, clinopyroxene coexists with plagioclase (An67), olivine (For8), and liquid.

Samples 396B-7-1, 42-46 cm and 396B-30-1, 77-80 cm have liquidus temperatures of about $1200^{\circ}$ to $1195^{\circ} \mathrm{C}$. In both of these samples, plagioclase is joined by olivine within 10 degrees of the temperature of appearance of the plagioclase. In Sample 396B-30-1, 77-80 cm, plagioclase (An81) appears at slightly above $1200^{\circ} \mathrm{C}$ and at $1175^{\circ} \mathrm{C}$ plagioclase (An73) coexists with olivine (Fo84) and liquid. Clinopyroxene appears at about $1170^{\circ} \mathrm{C}$; at $1150^{\circ} \mathrm{C}$, plagioclase (An65), olivine (Fo80), pyroxene, and liquid all coexist.

In Sample 396B-7-1, 42-46 cm, plagioclase is estimated to first appear at about $1195^{\circ} \mathrm{C}$; by $1175^{\circ} \mathrm{C}$, plagioclase 
$\left(\mathrm{An}_{74}\right)$ and olivine (Fo84) coexist with liquid. At $1150^{\circ} \mathrm{C}$, plagioclase $\left(\mathrm{An}_{67}\right)$, olivine (Fo81), and clinopyroxene all coexist with liquid.

The compositions of three experimentally produced chromian spinels in $1200^{\circ}$ and $1250^{\circ} \mathrm{C}$ runs with Sample 396B-22-3, 28-38 cm are plotted on Figure 7. Their $\mathrm{Cr} / \mathrm{Cr}+\mathrm{Al}$ and $\mathrm{Mg} / \mathrm{Mg}+\mathrm{Fe}$ ratios agree well with those in the natural rock and, furthermore, they show a consistent variation with temperature which in turn mimics compositional trends in the natural spinels.

\section{Cooling Rate Experiments}

The linear cooling rate experiments for Sample $396 \mathrm{~B}-22-3,28-38 \mathrm{~cm}$ produced textures ranging from porphyritic at $2 \% \mathrm{hr}$ to fan-spherulitic at $30 \% \mathrm{hr}$. In the $2 \% \mathrm{hr}$ run, slightly skeletal, tabular plagioclase laths up to $3 \mathrm{~mm}$ in length by $0.2 \mathrm{~mm}$ wide are set in a groundmass of much finer grained plagioclase laths, subhedral equant olivine grains $(<0.2 \mathrm{~mm})$, and sheaf-like aggregates of fine-grained acicular clinopyroxene. Wedges and laths of titanomagnetite are common throughout the charge and, in some parts of it, they show a peculiar subparallel alignment roughly perpendicular to the largest face of a plagioclase lath.

A cooling rate of $10 \% \mathrm{hr}$ produced a texture that is similar to that of the $2 \% \mathrm{hr}$ run, but the plagioclase laths are thinner and are partly fan-spherulitic. In addition, there are very few equant olivine grains. Instead, they are either very thin-chained olivines oriented subparallel to plagioclase laths or they occur as very fine-grained spherulitic aggregates. Clinopyroxene also occurs as fine-grained spherulitic aggregates, some of which appear to be partly intergrown with the margins of plagioclase laths.

The $30 \%$ hr run produced a texture in which plagioclase is again the dominant mineral, but it occurs as radiating aggregates of acicular, partly skeletal grains which appear in transmitted light to merge with a fine-grained groundmass. In reflected light, it is obvious that olivine and/or pyroxene grains are arranged perpendicular to surfaces of plagioclase laths, imparting a dendritic appearance to them. The only truly dendritic crystals are, however, some of the groundmass olivines.

The textural changes in Sample 396B-22-3, 28-38 cm with increasing cooling rate can be summarized as follows: (1) plagioclase becomes more acicular and fan-spherulitic, (2) grain boundaries of the larger plagioclases become less and less distinct, and (3) olivine morphology changes from sub-equant to chain-like to dendritic. There is also a general decrease in groundmass grain size.

Cooling rate experiments for Sample 396B-30-1, 77-80 $\mathrm{cm}$ produced only olivine plus residual glass. The olivine morphologies and the proportions of crystals to glass, however, change systematically with cooling rate. At $2 \% \mathrm{hr}$ euhedral, chained olivine crystals are distributed uniformly throughout the charge. They are sub-equant and partly skeletal and make up 7 to 15 per cent of the charge (visual estimate) with a grain size of about 0.1 to $0.2 \mathrm{~mm}$. In some parts of the charge, the chain-links ofolivine are arranged like stylized tree branches.

The $10 \%$ hr run produced numerous pinwheels of olivine dendrites which in cross-section appear to be chained olivines. The average diameter of the pinwheels is less than a millimeter and their thickness is about $0.05 \mathrm{~mm}$. Olivine makes up no more than 5 per cent of the charge.

The $30^{\circ} / \mathrm{hr}$ charge is grossly similar to the $10^{\circ} / \mathrm{hr}$ one, but the olivine pinwheels are less abundant and are smaller. The pinwheels are more plumose and each one consists of several subparallel dendrite stems which are generally less than $0.5 \mathrm{~mm}$ in length.

Sample 396B-7-1, 42-46 cm, when cooled at $2 \% \mathrm{hr}$, produced radiating acicular plagioclase $(<0.8 \mathrm{~mm})$ interspersed with granular to elongate olivine grains $\{0.5$ $\mathrm{mm}$ ) and very fine grained plumose clinopyroxene. Tabular titanomagnetite grains are also present; they commonly are approximately perpendicular to the long dimension of the plagioclase grains. The $10^{\circ} / \mathrm{hr}$ run for Sample 396B-7-1, $42-46 \mathrm{~cm}$, however, looks very much like the $10 \% \mathrm{hr}$ run for Sample 396B-30-1, 77-80 cm. The main difference is that less olivine is present in Sample 396B-7-1, 42-46 cm. A similar similarity occurs at $30^{\circ} / \mathrm{hr}$. At this cooling rate, Sample 396B-7-1, $42-46 \mathrm{~cm}$ produced only a few compact flattened ovoids of fine-grained olivine dendrites possessing pinwheel geometry.

Comparison of textures produced in the cooling rate experiments on thin sections of Sample 396B-22-3, 28-38 $\mathrm{cm}$ with other samples of similar composition suggest a range of cooling rates from about $2^{\circ}$ to $5^{\circ} / \mathrm{hr}$ for the interior parts of pillows. This estimate is based on the groundmass textures of the rocks and assumes (1) that phenocrysts present at eruption did not exert a profound effect on groundmass textures and (2) that there was no time lag for nucleation of plagioclase in the experimental runs. The latter assumption is probably valid since some plagioclase was present at the start of the drop and this could serve as nucleation sites.

Direct interpretation of the cooling rate experiments for Samples 396B-7-1, $42-46 \mathrm{~cm}$ and 396B-30-1, 77-80 cm is difficult because both of these samples have a much lower liquidus than 396B-22-3, 28-38 cm; all three samples were cooled simultaneously in the same furnace. Thus, Samples 396B-7-1, 42-46 cm and 396B-30-1, 77-80 cm were superheated about $50^{\circ} \mathrm{C}$ at the start of the cooling rate experiments. This is reflected in the suppression of plagioclase nucleation in Sample 396B-30-1, 77-80 cm even at $2 \%$ hr. This suggests that the ability of the melt to re-equilibrate in response to decreasing temperature is even slower than $2 \% \mathrm{hr}$ and that the effective supercooling of the melt probably increases continuously during the cooling rate experiments. Olivine morphologies are controlled in part by the degree of supercooling as shown by Donaldson (1976), and these experiments suggest that the nucleation delay caused by superheat may result in greater undercoolings at the time of olivine growth. Thus, if there are any naturally superheated basaltic magmas, this presents an additional variable to be considered in the interpretation of olivine morphologies as indicators of cooling rate.

\section{DISCUSSION}

The chondrite-normalized rare-earth abundances for the Site 396 samples (Figure 2) show them to be "normal" LIL element-depleted basalts. They are light-rare earth depleted, with $\mathrm{La} / \mathrm{Sm}$ and $\mathrm{La} / \mathrm{Yb}$ ratios less than 1.0 , similar to the 
Type I basalts of Bryan et al. (1976). The $\mathrm{Nb}$ content of these basalts is low, resulting in high $\mathrm{Zr} / \mathrm{Nb}$ ratios similar to those observed in other LIL element-depleted basalts (Pearce and Cann, 1973; Erlank and Kable, 1976; Rhodes et al., 1976), and considerably higher than the $\mathrm{Zr} / \mathrm{Nb}$ ratios $(<10)$ of oceanic islands and "anomalous" Type II basalts sampled on the Mid-Atlantic Ridge at $45^{\circ} \mathrm{N}$ and in the FAMOUS area (Erlank and Kable, 1976; Rhodes et al., unpublished data).

The chemical differences among the residual glasses of the various units at Site 396 preclude their being related by fractional crystallization of the observed phenocrysts. On the other hand, the trace element ratios in Sub-units $\mathrm{A}_{1}, \mathrm{~A}_{2}$, and $A_{3}$ and in Unit $B$ are quite similar and vary in a general way that is compatible with small amounts of crystal fractionation. An obvious exception is the ratio of $\mathrm{Sr}$ to other magmaphile elements. This is illustrated by the $\mathrm{Sr} / \mathrm{Zr}$ ratio, which is relatively constant in the aphyric units where $\mathrm{Sr}$ tends to behave as a magmaphile element, but is both distinctly different and more variable within Unit B where the irregular distribution of plagioclase exerts a partial control. This effect is less marked at Site 396 than in the phyric basalts at Site 395 , where $\mathrm{CaO}$ and $\mathrm{Al}_{2} \mathrm{O}_{3}$ variations indicate much more heterogeneity in the content of plagioclase phenocrysts. Despite these small differences, we deduce that these ratios approximately reflect the characteristics of the mantle source region, and that the basalts of Units A and B were derived from an essentially similar and homogeneous LIL-element depleted mantle source. Similar ratios are observed in basalts from Holes 395 (Table 2) and 395A (Rhodes et al., 1978), implying a widespread and homogeneous mantle source for basaltic volcanism along this section of the Mid-Atlantic Ridge. This contrasts markedly with the wide variations in magmaphile element ratios found in Leg 37 basalts, both within and between holes (Blanchard et al., 1976), and the apparent necessity to postulate a complex and heterogeneous mantle source for the basalts in that region.

Although these basalts appear ultimately to have been derived by partial melting of a common source, all are evolved and have undergone moderate amounts of differentiation. Evidence of this is provided by the low $\mathrm{Mg}^{\prime}$ values (0.59-0.69), low $\mathrm{Ni}$ concentrations $(<180 \mathrm{ppm})$, moderate magmaphile element abundances (e.g., $\mathrm{TiO}_{2}=$ 0.95 to $1.6 \%$ ), and the presence of multiple phenocryst phases in all the basalts. Since the compositional variations within the individual basalt groups is small, we suggest that the magma compositions were established by differentiation within shallow magma chambers, followed by episodic eruption onto the sea floor, with little or no fractionation at the surface or enroute to the surface.

There can be little doubt that the compositions of the glassy and variolitic aphyric basalts are close to those of magmatic liquids. The compositions of the phyric basalts are more enigmatic. Several lines of evidence indicate that these rocks are far removed from liquid compositions:

a) Compilations of basaltic glass compositions (Melson et al., 1976) indicate that magmatic liquids with more than 17 per cent $\mathrm{Al}_{2} \mathrm{O}_{3}$ are extremely rare. Similarly, the glassy selvages on the phyric basalt pillows are much lower in $\mathrm{Al}_{2} \mathrm{O}_{3}$ and not substantially different from the aphyric basalts (Figure 4).

b) The wide scatter of compositions in the normative olivine-plagioclase-pyroxene diagram (Figure 1) is suggestive of plagioclase and olivine addition to liquids close to the inferred olivine-plagioclase cotectic.

c) Experimental, textural and compositional evidence indicates that the most magnesian olivine phenocrysts in the phyric basalts are too forsteritic to have crystallized from melts having the compositions of the phyric basalts, but are derived instead from more "primitive" basalts.

d) Glass melt inclusions within olivine and plagioclase phenocrysts record entrapment of this more primitive liquid and provide insight into its composition. Its general characteristics are high $\mathrm{CaO} / \mathrm{Al}_{2} \mathrm{O}_{3}(>0.85)$, low $\mathrm{TiO}_{2}$ $(<0.80 \mathrm{wt} \%)$, and high $\mathrm{Mg}^{\prime}(>0.70)$.

e) The trend of decreasing $\mathrm{CaO} / \mathrm{Al}_{2} \mathrm{O}_{3}$ with increasing $\mathrm{TiO}_{2}$ and decreasing $\mathrm{Mg}^{\prime}$ among related residual glasses implies that clinopyroxene fractionation plays a role in the overall differentiation scheme despite its absence as a phenocryst at Site 396. The geochemical imprint of the clinopyroxene fractionation may be imparted to the observed residual liquids by mixing of a low $\mathrm{CaO} / \mathrm{Al}_{2} \mathrm{O}_{3}$ evolved magma with primitive liquids characterized by higher $\mathrm{CaO} / \mathrm{Al}_{2} \mathrm{O}_{3}$.

From this evidence, we think that the phyric basalts have a mixed heritage, resulting from mixing of consanguineous "primitive" and evolved magmas and their attendant phenocrysts. If this interpretation is correct, the higher $\mathrm{Mg}^{\prime}$-values, normative $\mathrm{An} /(\mathrm{Ab}+\mathrm{An})$ ratios, and lower magmaphile element abundances of the phyric basalts relative to the aphyric ones are due in part to the addition of up to 20 per cent plagioclase and 5 per cent olivine phenocrysts. The glassy rims on phyric basalt pillows probably provide the most reliable of the melt compositions. They have $\mathrm{Mg}^{\prime}$ values comparable to those of the more evolved aphyric basalts ( 0.60 to 0.63$)$, but are distinguished from them by lower $\mathrm{TiO}_{2}$ values (Figure 2) and by higher $\mathrm{CaO} / \mathrm{Al}_{2} \mathrm{O}_{3}$ ratios. Thus, the phyric basalts are not simply related to any of the aphyric basalt types by processes of plagioclase and olivine accumulation.

\section{CONCLUSIONS}

Thus, we have several discrete magma types at Site 396, both phyric and aphyric, that are not related directly by crystal fractionation, but which appear to have been derived from similar mantle source materials. The prevailing interpretation of such relationships is that each basalt type represents an evolved derivative of more "primitive" basaltic magmas that resulted from varying amounts of melting. The evidence, presented here, of magma mixing in the phyric basalts suggests that such a model may be unduly simple. We suggest instead that magma mixing of consanguineous evolved and "primitive" basalts explains many of the characteristics of typical ocean floor tholeiites. A subsequent publication documenting the role of magma mixing in these and other ocean floor basalts is in preparation. 


\section{REFERENCES}

Bass, M.N., 1971. Variable abyssal basalt populations and their relation to sea-floor spreading rates, Earth Planet. Sci. Lett., v. 11 , p. $18-22$.

Blanchard, D.P., Rhodes, J.M., Dungan, M.A., Rodgers, K.V., Donaldson, C.H., Brannon, J.C., Jacobs, J.W., and Gibson, E.K., 1976. The chemistry and petrology of basalts from Leg 37 of the Deep Sea Drilling Project, J. Geophys. Res., v. 81, p. 4231-4246.

Bryan, W.B. and Moore, J.G., 1976. Compositional variations of young basalts in the Mid-Atlantic Ridge rift valley near lat. $46^{\circ} 49^{\prime}$ N, Geol. Soc. Am. Bull., v. 88, p. 556-570.

Bryan, W.B., Thompson, G., Frey, F.A., and Dickey, J.J., 1976. Inferred settings and differentiation in basalts from the Deep Sea Drilling Project, J. Geophys. Res., v. 81, p. 4285-4304.

Donaldson, C.H., 1976. An experimental investigation of olivine morphology, Contrib. Mineral. Petrol., v. 57, p. 187-213.

Dungan, M.A., Long, P.E., and Rhodes, J.M., 1978. The petrography, mineral chemistry and one-atmosphere phase relations of basalts from Site 395-Leg 45 DSDP. In Melson, W.G., Rabinowitz, P.D., et al., Initial Reports of the Deep Sea Drilling Project, v. 45: Washington (U.S. Government Printing Office).

Engel, A.E., Engel, C.G., and Havens, R.G., 1965. Chemical characteristics of oceanic basalts and the upper mantle. Geol. Soc. Am. Bull., v. 76, p. 719-734.

Erlank, A.J. and Kable, E.J.D., 1976. The significance of incompatible elements in mid-Atlantic ridge basalts from $45^{\circ} \mathrm{N}$ with particular reference to $\mathrm{Zr} / \mathrm{Nb}$, Contrib. Mineral. Petrol., v. 54 , p. $281-291$.

Frey, F.A., Bryan, W.B., and Thompson, G., 1974. Atlantic ocean floor: Geochemistry and petrology of basalts from Legs 2 and 3 of the Deep Sea Drilling Project, J. Geophys. Res. v. 79, p. 5507-5527.

Fujii, T., Kushiro, I., and Hamuro, K., 1978. Melting relations and viscosity of an abyssal olivine tholeiite. In Melson, W.G., Rabinowitz, P.D., et al., Initial Reports of the Deep Sea Drilling Project, v. 45. Washington (U.S. Government Printing Office)

Hart, S.R., 1976. LIL-element geochemistry, Leg 34 basalts. In Hart, S.R., Yeats, R.S., et al., Initial Reports of the Deep Sea Drilling Project, v. 34: Washington (U.S. Government Printing Office), p. 283-288.

Jacobs, J.W., Korotev, R.L., Blanchard, D.P., and Haskin, L.A., in press. A well tested procedure for instrumental neutron activation analysis of silicate rocks and minerals, J. Radioanal. Chem.

Kay, R., Hubbard, N.J., and Gast, P.W., 1970. Chemical characteristics and origin of oceanic ridge volcanic rocks, $J$. Geophys. Res., v. 75, p. 1585-1613.

Maxwell, J.A., 1968. Rock and mineral analysis: New York (Interscience).
Melson, W.G., Vallier, T.L., Wright, T.L., Byerly, G., and Nelen, J., 1976. Chemical diversity of abyssal volcanic glass erupted along Pacific, Atlantic, and Indian ocean sea-floor spreading centers. In The geophysics of the Pacific ocean basin and its margin: Washington (American Geophysical Union), p. 351-367.

Moore J.G. and Fabbi, B.P., 1971. An estimate of the juvenile sulfur content of basalt, Contrib. Mineral. Petrol., v. 33, p. 118-127.

Norrish, K. and Chappell, B.W., 1967. X-ray fluorescence spectrography. In Zussman, J. (Ed.), Physical methods in determinative mineralogy: New York (Academic Press), p. 161-214.

Norrish, K. and Hutton, J.T., 1969. An accurate X-ray spectrographic method for the analysis of a wide range of geological samples, Geochim. Cosmochim. Acta, v. 33, p. 431-453.

Pearce, J.A. and Cann, J.R., 1973. Tectonic setting of basic volcanic rocks determined using trace element analyses. Earth Planet. Sci. Lett., v. 19, p. 290-300.

Reynolds, R.C., 1967. Estimation of mass absorption coefficients by Compton scattering: Improvements and extensions of the method, Am. Mineral., v. 48, p. 1133-1143.

Rhodes, J.M., Blanchard, D.P., Rodgers, K.V., Jacobs, J.W., and Brannon, J.C., 1976. Petrology and chemistry of basalts from the Nazca plate: Part 2 - major and trace element chemistry. In Hart, S.R., Yeats, R.S., et al., Initial Reports of the Deep Sea Drilling Project, v.34: Washington (U.S. Government Printing Office), p. 239-244.

Rhodes, J.M., Blanchard, D.P., Dungan, M.A., Rodgers, K.V., and Brannon, J.C., 1978. Chemistry of basalts from Leg 45 of the Deep Sea Drilling Project. In Melson, W.G., Rabinowitz, P.D., et al., Initial Reports of the Deep Sea Drilling Project, v. 45: Washington (U.S. Government Printing Office).

Roeder, P.L. and Emslie, P.F., 1970. Olivine-liquid equilibrium, Contrib. Mineral. Petrol, v. 29, p. 275-289.

Schilling, J.G., 1971. Sea-floor evolution: Rare-earth evidence, Phil. Trans. Roy. Soc. London, Ser. A, v. 268, p. 663-706.

, 1975. Rare earth variations across 'normal segments' of the Reykjanes Ridge, $60^{\circ}-53^{\circ} \mathrm{N}$, Mid-Atlantic Ridge, $29^{\circ} \mathrm{S}$, and East Pacific Rise $2^{\circ}-19^{\circ} \mathrm{S}$, and evidence on the composition of the underlying low velocity layer, J. Geophys. Res., v. 80. p. 1459-1473.

Shido, F.A., Miyashiro, A., and Ewing, M., 1971. Crystallization of abyssal tholeiites, Contrib. Mineral. Petrol., v.31, p. 251-266.

Sigurdsson, H. and Schilling, J.G., 1976. Spinels in mid-Atlantic ridge basalts; chemistry and occurrence, Earth Planet. Sci. Lett., v. 29, p. 720.

Yoder, H.S. and Tilley, C.E., 1972. Origin of basalt magmas: an experimental study of natural and synthetic rock systems, $J$. Petrol. v. 3, p. 342-532. 